NIST Special Publication 800-90A Revision 1

\title{
Recommendation for Random Number Generation Using Deterministic Random Bit Generators
}

Elaine Barker John Kelsey

This publication is available free of charge from: http://dx.doi.org/10.6028/NIST.SP.800-90Ar1

\section{O M P U TER SE C U R I T Y}


NIST Special Publication 800-90A

Revision 1

\title{
Recommendation for Random Number Generation Using Deterministic Random Bit Generators
}

\author{
Elaine Barker \\ John Kelsey \\ Computer Security Division \\ Information Technology Laboratory
}

This publication is available free of charge from: http://dx.doi.org/10.6028/NIST.SP.800-90Ar1

June 2015

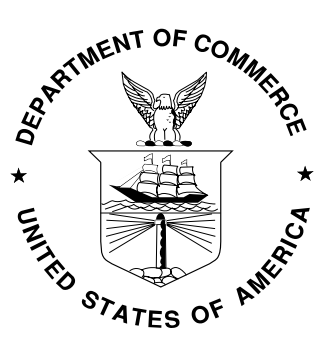

U.S. Department of Commerce

Penny Pritzker, Secretary

National Institute of Standards and Technology

Willie May, Under Secretary of Commerce for Standards and Technology and Director 


\section{Authority}

This publication has been developed by NIST to further its statutory responsibilities under the Federal Information Security Modernization Act (FISMA) of 2014, 44 U.S.C. $§ 3541$ et seq., Public Law (P.L.) 113-283. NIST is responsible for developing information security standards and guidelines, including minimum requirements for Federal information systems, but such standards and guidelines shall not apply to national security systems without the express approval of appropriate Federal officials exercising policy authority over such systems. This guideline is consistent with the requirements of the Office of Management and Budget (OMB) Circular A-130, Section 8b(3), Securing Agency Information Systems, as analyzed in Circular A-130, Appendix IV: Analysis of Key Sections. Supplemental information is provided in Circular A-130, Appendix III, Security of Federal Automated Information Resources.

Nothing in this publication should be taken to contradict the standards and guidelines made mandatory and binding on Federal agencies by the Secretary of Commerce under statutory authority. Nor should these guidelines be interpreted as altering or superseding the existing authorities of the Secretary of Commerce, Director of the OMB, or any other Federal official. This publication may be used by nongovernmental organizations on a voluntary basis and is not subject to copyright in the United States. Attribution would, however, be appreciated by NIST.

\section{National Institute of Standards and Technology Special Publication 800-90A Revision 1 Natl. Inst. Stand. Technol. Spec. Publ. 800-90A Rev. 1, 109 pages (June 2015) CODEN: NSPUE2}

This publication is available free of charge from: http://dx.doi.org/10.6028/NIST.SP.800-90Ar1

Certain commercial entities, equipment, or materials may be identified in this document in order to describe an experimental procedure or concept adequately. Such identification is not intended to imply recommendation or endorsement by NIST, nor is it intended to imply that the entities, materials, or equipment are necessarily the best available for the purpose.

There may be references in this publication to other publications currently under development by NIST in accordance with its assigned statutory responsibilities. The information in this publication, including concepts and methodologies, may be used by Federal agencies even before the completion of such companion publications. Thus, until each publication is completed, current requirements, guidelines, and procedures, where they exist, remain operative. For planning and transition purposes, Federal agencies may wish to closely follow the development of these new publications by NIST.

Organizations are encouraged to review all draft publications during public comment periods and provide feedback to NIST. All NIST Computer Security Division publications, other than the ones noted above, are available at http://csrc.nist.gov/publications.

\section{Comments may be provided to:}

National Institute of Standards and Technology Attn: Computer Security Division, Information Technology Laboratory 100 Bureau Drive (Mail Stop 8930) Gaithersburg, MD 20899-8930

Email: rbg_comments@nist.gov 


\title{
Reports on Computer Systems Technology
}

The Information Technology Laboratory (ITL) at the National Institute of Standards and Technology (NIST) promotes the U.S. economy and public welfare by providing technical leadership for the Nation's measurement and standards infrastructure. ITL develops tests, test methods, reference data, proof of concept implementations, and technical analyses to advance the development and productive use of information technology. ITL's responsibilities include the development of management, administrative, technical, and physical standards and guidelines for the cost-effective security and privacy of other than national security-related information in Federal information systems. The Special Publication 800-series reports on ITL's research, guidelines, and outreach efforts in information system security, and its collaborative activities with industry, government, and academic organizations.

\begin{abstract}
This Recommendation specifies mechanisms for the generation of random bits using deterministic methods. The methods provided are based on either hash functions or block cipher algorithms.
\end{abstract}

\section{Keywords}

Deterministic random bit generator (DRBG); entropy; hash function; random number generator.

\section{Acknowledgements}

The National Institute of Standards and Technology (NIST) gratefully acknowledges and appreciates contributions by Mike Boyle and Mary Baish from NSA for assistance in the development of this Recommendation. NIST also thanks the many contributions by the public and private sectors. 


\section{Table of Contents}

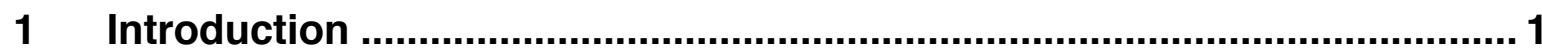

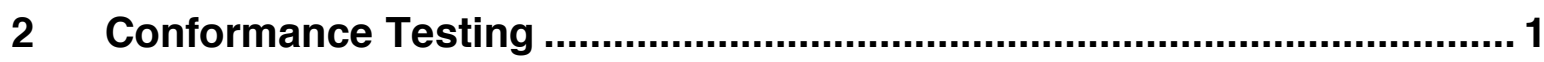

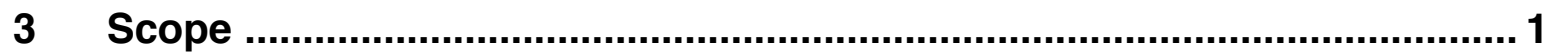

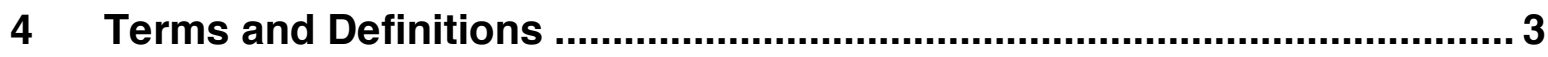

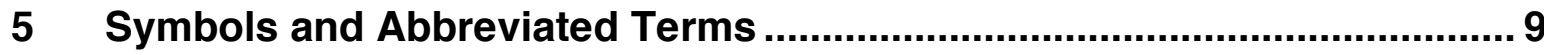

6 Document Organization ........................................................................ 10

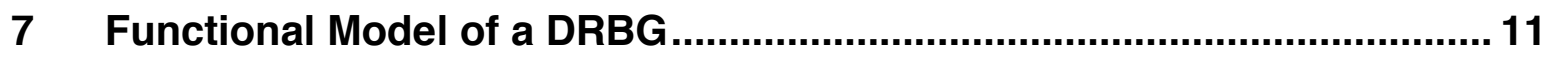

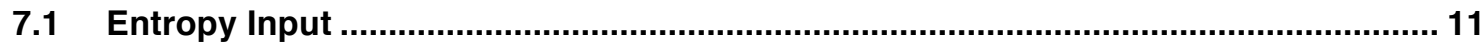

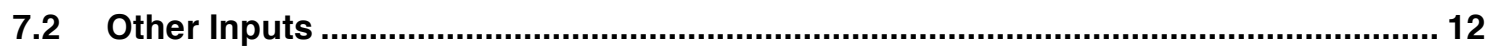

7.3 The Internal State ............................................................................................ 12

7.4 The DRBG Mechanism Functions ..................................................................... 12

8. DRBG Mechanism Concepts and General Requirements......................13

8.1 DRBG Mechanism Functions ............................................................................. 13

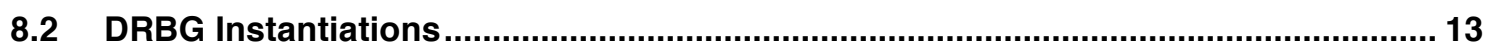

8.3 Internal States................................................................................................... 13

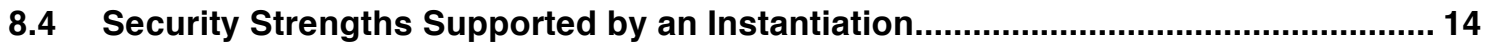

8.5 DRBG Mechanism Boundaries.............................................................................. 15

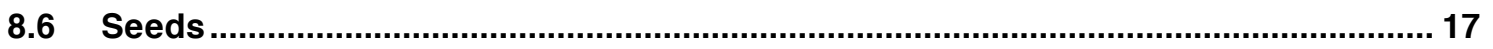

8.6.1 Seed Construction for Instantiation ............................................................ 18

8.6.2 Seed Construction for Reseeding ……....................................................... 18

8.6.3 Entropy Requirements for the Entropy Input ……...................................... 18

8.6.4 Seed Length ............................................................................................... 19

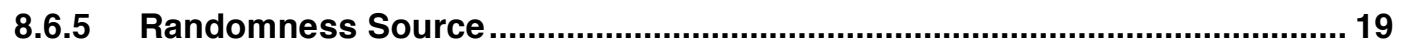

8.6.6 Entropy Input and Seed Privacy .............................................................. 19

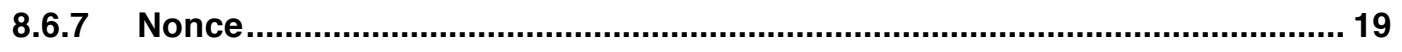

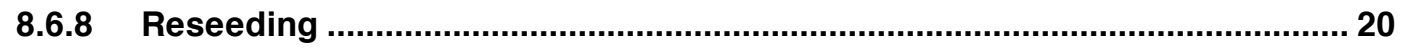

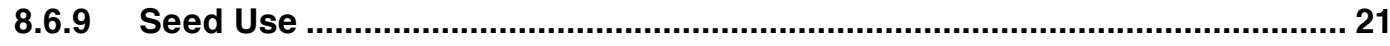

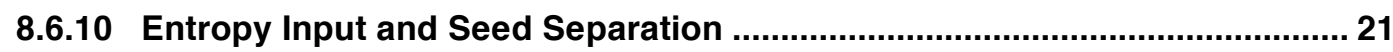

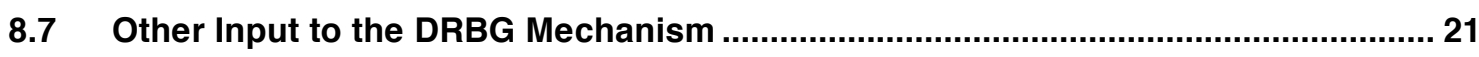

8.7.1 Personalization String................................................................................. 21

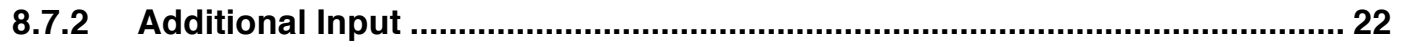




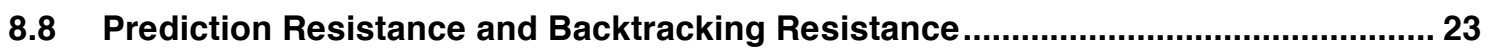

9 DRBG Mechanism Functions ............................................................. 25

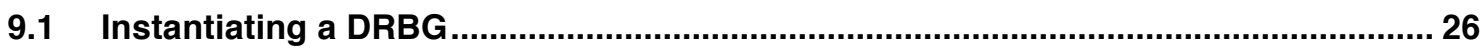

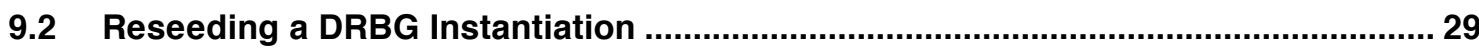

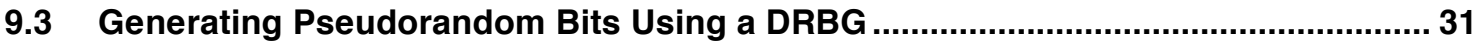

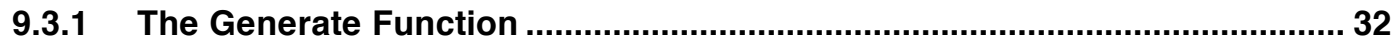

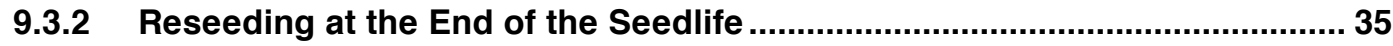

9.3.3 Handling Prediction Resistance Requests .............................................. 35

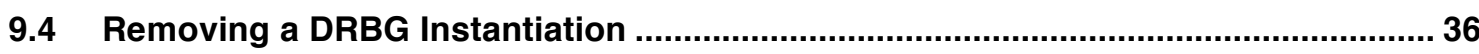

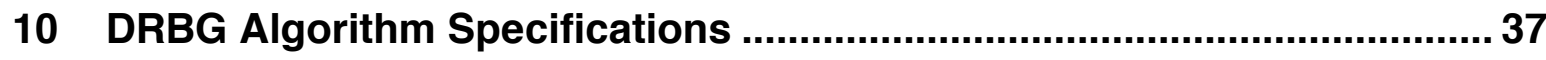

10.1 DRBG Mechanisms Based on Hash Functions .................................................... 37

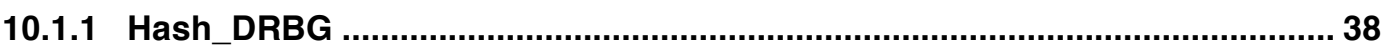

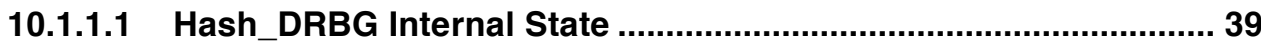

10.1.1.2 Instantiation of Hash_DRBG ..................................................... 39

10.1.1.3 Reseeding a Hash_DRBG Instantiation ...................................... 40

10.1.1.4 Generating Pseudorandom Bits Using Hash_DRBG ................... 41

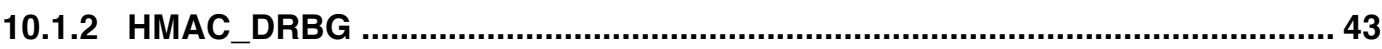

10.1.2.1 HMAC_DRBG Internal State ........................................................ 43

10.1.2.2 The HMAC_DRBG Update Function (Update)..............................4 44

10.1.2.3 Instantiation of HMAC_DRBG .................................................... 45

10.1.2.4 Reseeding an HMAC_DRBG Instantiation .................................. 46

10.1.2.5 Generating Pseudorandom Bits Using HMAC_DRBG ................. 46

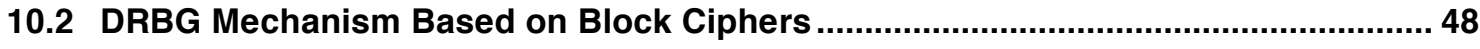

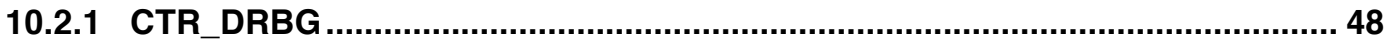

10.2.1.1 CTR_DRBG Internal State ........................................................... 50

10.2.1.2 The Update Function (CTR_DRBG_Update) .................................51

10.2.1.3 Instantiation of CTR_DRBG ...................................................... 52

10.2.1.4 Reseeding a CTR_DRBG Instantiation ........................................ 54

10.2.1.5 Generating Pseudorandom Bits Using CTR_DRBG .................... 55

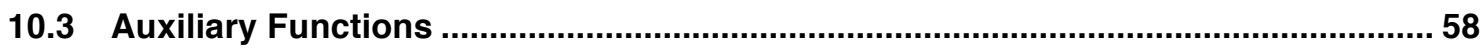

10.3.1 Derivation Function Using a Hash Function (Hash_df) .................................58

10.3.2 Derivation Function Using a Block Cipher Algorithm (Block_Cipher_df).... 59 


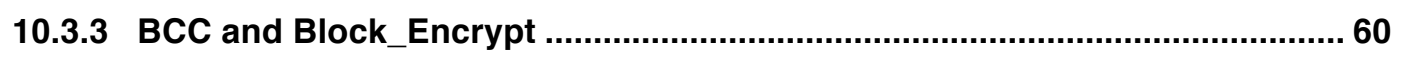

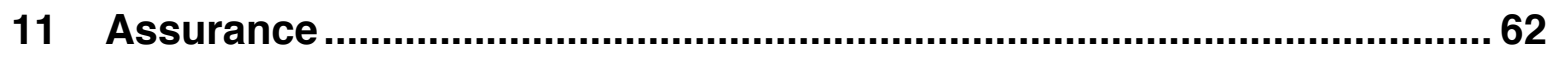

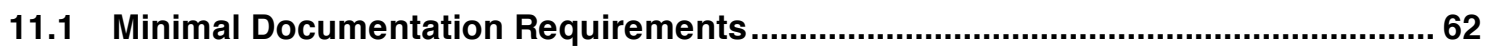

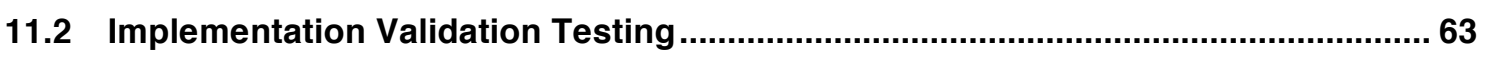

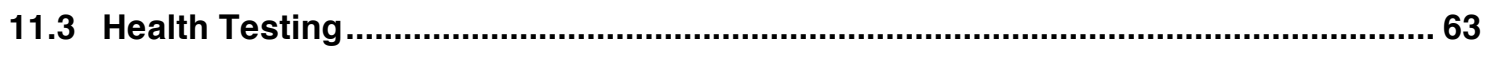

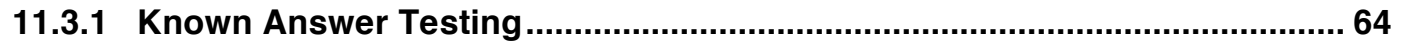

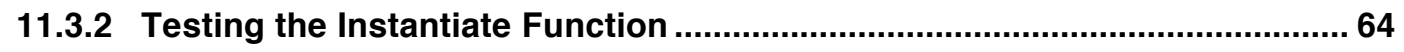

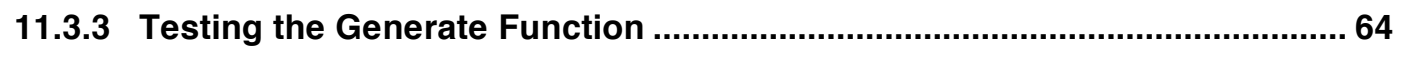

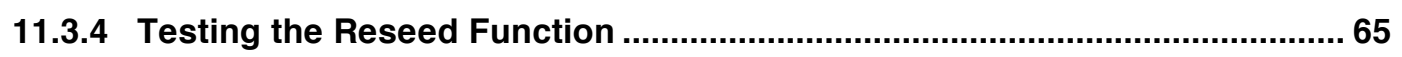

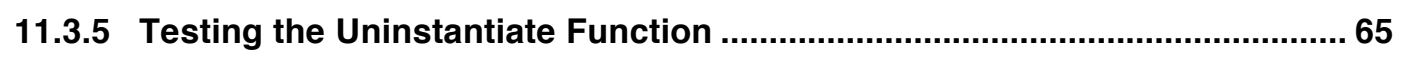

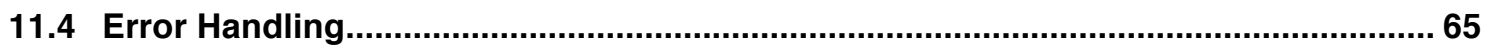

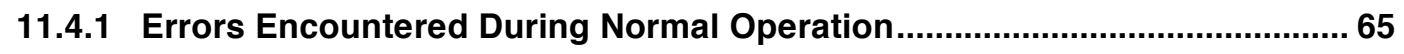

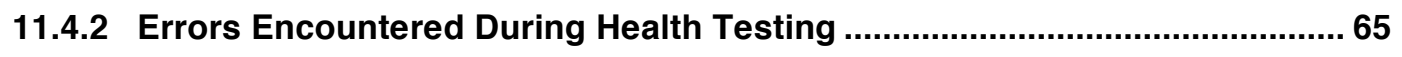

Appendix A: (Normative) Conversion and Auxiliary Routines ......................67 67

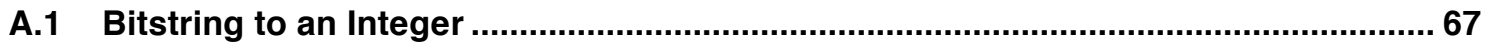

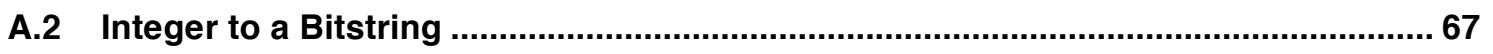

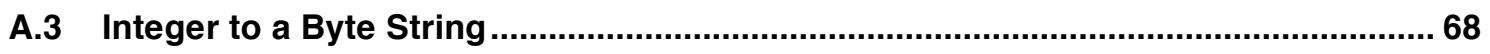

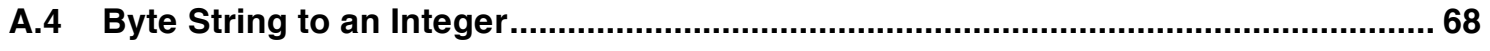

A.5 Converting Random Bits into a Random Number ................................................. 68

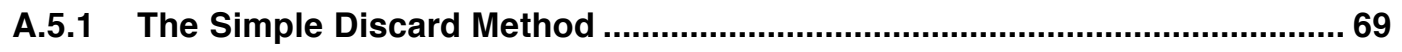

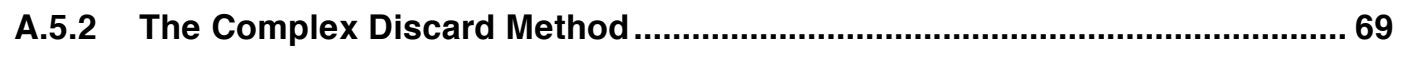

A.5.3 The Simple Modular Method ................................................................. 70

Appendix B: (Informative) Example Pseudocode for Each DRBG Mechanism

71

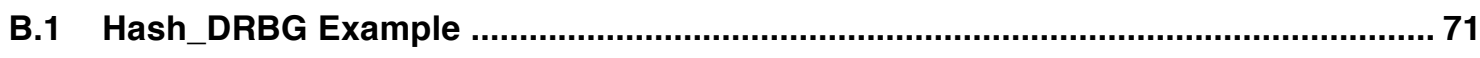

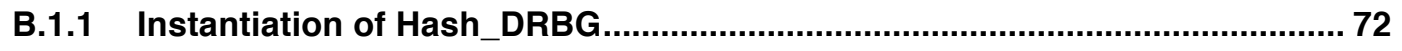

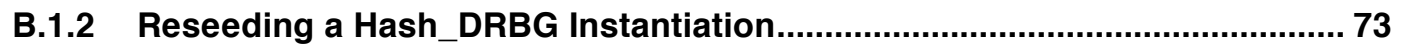

B.1.3 Generating Pseudorandom Bits Using Hash_DRBG ................................ 74

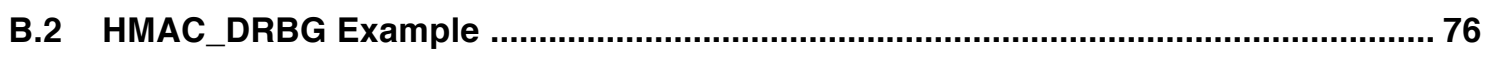

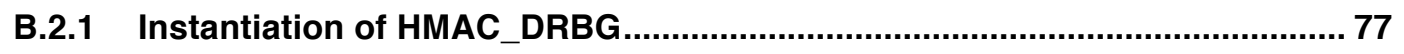

B.2.2 Generating Pseudorandom Bits Using HMAC_DRBG .................................... 78

B.3 CTR_DRBG Example Using a Derivation Function ............................................. 80

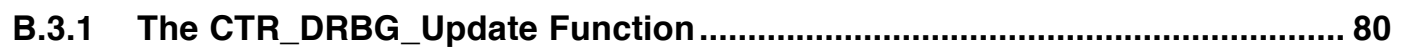


B.3.2 Instantiation of CTR_DRBG Using a Derivation Function ........................... 81

B.3.3 Reseeding a CTR_DRBG Instantiation Using a Derivation Function ........... 82

B.3.4 Generating Pseudorandom Bits Using CTR_DRBG ..................................... 84

B.4 CTR_DRBG Example Without a Derivation Function .................................................. 86

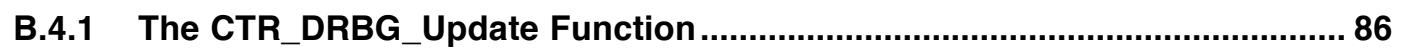

B.4.2 Instantiation of CTR_DRBG Without a Derivation Function ............................ 86

B.4.3 Reseeding a CTR_DRBG Instantiation Without a Derivation Function........ 86

B.4.4 Generating Pseudorandom Bits Using CTR_DRBG.................................... 87

Appendix C: (Informative) DRBG Mechanism Selection ................................88

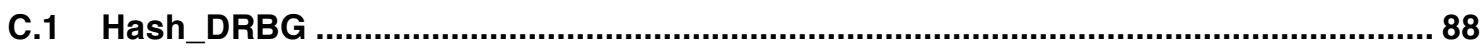

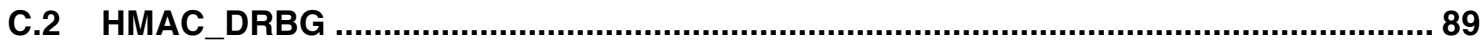

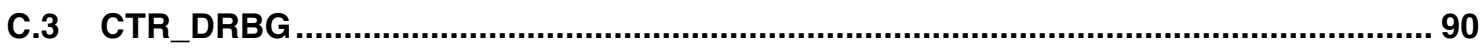

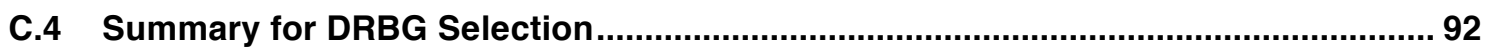

Appendix D : (Informative) References............................................................ 93

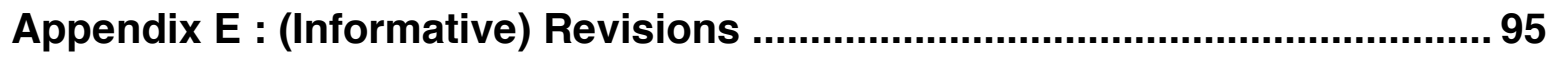

\section{List of Figures}

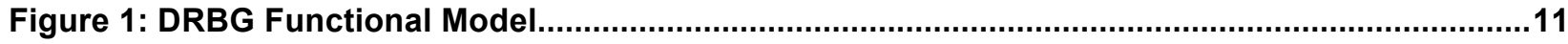

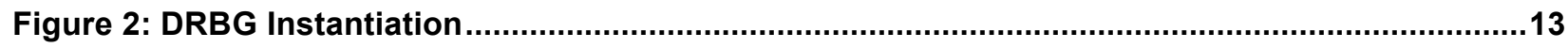

Figure 3: DRBG Mechanism Functions within a Single Device.......................................................16

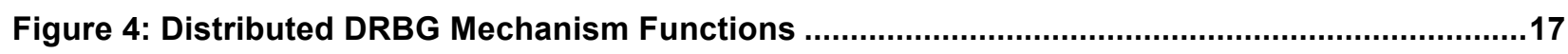

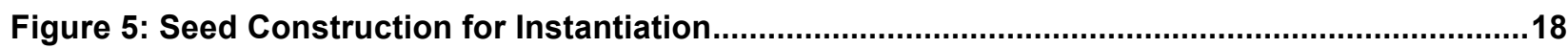

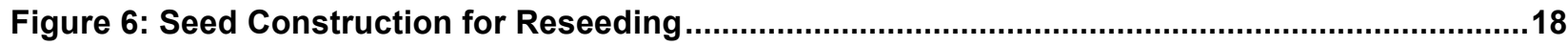

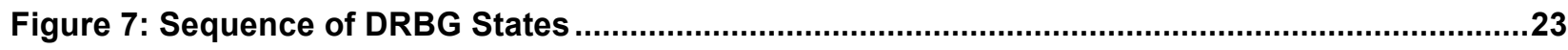

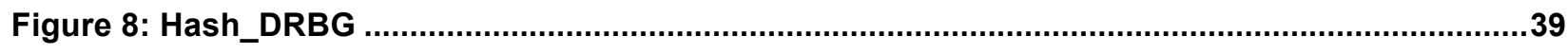

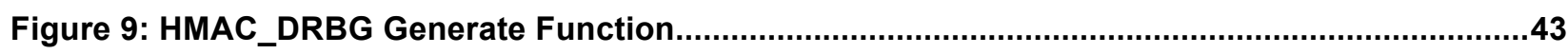

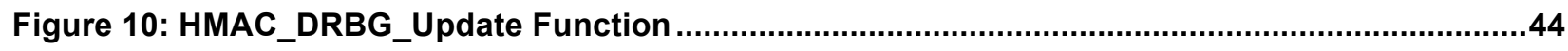

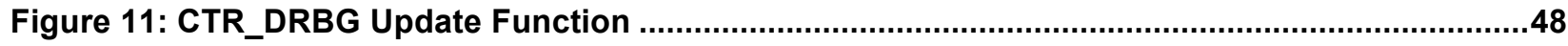

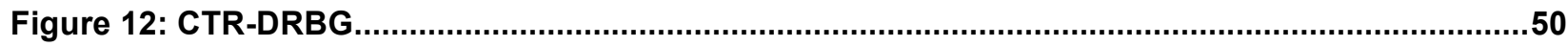

\section{List of Tables}

Table 1: Possible Instantiated Security Strengths ..........................................................................14

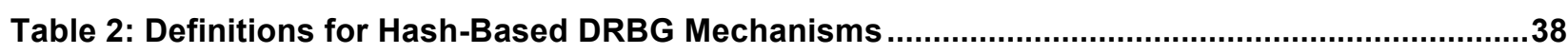

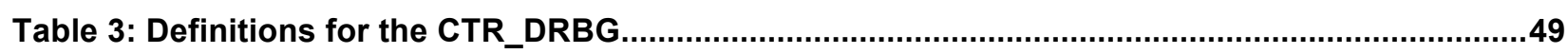


Table C-1: DRBG Mechanism Summary. 


\section{Introduction}

This Recommendation specifies techniques for the generation of random bits that may then be used directly or converted to random numbers when random values are required by applications using cryptography.

There are two fundamentally different strategies for generating random bits. One strategy is to produce bits non-deterministically, where every bit of output is based on a physical process that is unpredictable; this class of random bit generators (RBGs) is commonly known as non-deterministic random bit generators (NRBGs) ${ }^{1}$. The other strategy is to compute bits deterministically using an algorithm; this class of RBGs is known as Deterministic Random Bit Generators (DRBGs) ${ }^{2}$.

A DRBG is based on a DRBG mechanism as specified in this Recommendation and includes a source of randomness. A DRBG mechanism uses an algorithm (i.e., a DRBG algorithm) that produces a sequence of bits from an initial value that is determined by a seed that is determined from the output of the randomness source. Once the seed is provided and the initial value is determined, the DRBG is said to be instantiated and may be used to produce output. Because of the deterministic nature of the process, a DRBG is said to produce pseudorandom bits, rather than random bits. The seed used to instantiate the DRBG must contain sufficient entropy to provide an assurance of randomness. If the seed is kept secret, and the algorithm is well designed, the bits output by the DRBG will be unpredictable, up to the instantiated security strength of the DRBG.

The security provided by an RBG that uses a DRBG mechanism is a system implementation issue; both the DRBG mechanism and its randomness source must be considered when determining whether the RBG is appropriate for use by consuming applications.

\section{Conformance Testing}

Conformance testing for implementations of this Recommendation will be conducted within the framework of the Cryptographic Module Validation Program (CMVP) and the Cryptographic Algorithm Validation Program (CAVP). The requirements of this Recommendation are indicated by the word "shall." Some of these requirements may be out-of-scope for CMVP or CAVP validation testing, and thus are the responsibility of entities using, implementing, installing or configuring applications that incorporate this Recommendation.

\section{Scope}

This Recommendation includes:

1. Requirements for the use of DRBG mechanisms,

2. Specifications for DRBG mechanisms that use hash functions and block ciphers,

3. Implementation issues, and

4. Assurance considerations.

\footnotetext{
${ }^{1}$ NRBGs have also been called True Random Number (or Bit) Generators or Hardware Random Number Generators.

2 DRBGS have also been called Pseudorandom Number (or Bit) Generators.
} 
This Recommendation specifies several DRBG mechanisms, all of which provided acceptable security when this Recommendation was published. However, in the event that new attacks are found on a particular class of DRBG mechanisms, a diversity of approved mechanisms will allow a timely transition to a different class of DRBG mechanism.

Random number generation does not require interoperability between two entities, i.e., communicating entities may use different DRBG mechanisms without affecting their ability to communicate. Therefore, an entity may choose a single, appropriate DRBG mechanism for their consuming applications; see Appendix C for a discussion of DRBG mechanism selection.

The precise structure, design and development of a random bit generator is outside the scope of this document.

NIST Special Publication (SP) 800-90B [SP 800-90B] provides guidance on designing and validating entropy sources. SP 800-90C [SP 800-90C] provides guidance on the construction of an RBG from a randomness source and an approved DRBG mechanism from this document (i.e., SP 800-90A). 


\section{$4 \quad$ Terms and Definitions}

\begin{tabular}{|c|c|}
\hline Algorithm & $\begin{array}{l}\text { A clearly specified mathematical process for computation; a } \\
\text { set of rules that, if followed, will give a prescribed result. }\end{array}$ \\
\hline Approved & $\begin{array}{l}\text { FIPS-approved, NIST-Recommended and/or validated by the } \\
\text { Cryptographic Algorithm Validation Program (CAVP). }\end{array}$ \\
\hline Approved entropy source & $\begin{array}{l}\text { An entropy source that has been validated as conforming to } \\
\text { [SP 800-90B]. }\end{array}$ \\
\hline Backtracking Resistance & $\begin{array}{l}\text { An RBG provides backtracking resistance relative to time } T \text { if } \\
\text { it provides assurance that an adversary that has knowledge of } \\
\text { the state of the RBG at some time(s) subsequent to time } T \text { (but } \\
\text { incapable of performing work that matches the claimed } \\
\text { security strength of the RBG) would be unable to distinguish } \\
\text { between observations of ideal random bitstrings and } \\
\text { (previously unseen) bitstrings that are output by the RBG at or } \\
\text { prior to time } T \text {. In particular, an RBG whose design allows the } \\
\text { adversary to "backtrack" from the initially-compromised RBG } \\
\text { state(s) to obtain knowledge of prior RBG states and the } \\
\text { corresponding outputs (including the RBG state and output at } \\
\text { time } T \text { ) would not provide backtracking resistance relative to } \\
\text { time } T \text {. (Contrast with prediction resistance.) }\end{array}$ \\
\hline Biased & $\begin{array}{l}\text { A value that is chosen from a sample space is said to be biased } \\
\text { if one value is more likely to be chosen than another value. } \\
\text { Contrast with unbiased. }\end{array}$ \\
\hline Bitstring & A bitstring is an ordered sequence of 0 's and 1's. \\
\hline Bitwise Exclusive-Or & $\begin{array}{l}\text { An operation on two bitstrings of equal length that combines } \\
\text { corresponding bits of each bitstring using an exclusive-or } \\
\text { operation. }\end{array}$ \\
\hline Block Cipher & $\begin{array}{l}\text { A symmetric-key cryptographic algorithm that transforms one } \\
\text { block of information at a time using a cryptographic key. For } \\
\text { a block cipher algorithm, the length of the input block is the } \\
\text { same as the length of the output block. }\end{array}$ \\
\hline Consuming Application & $\begin{array}{l}\text { The application (including middleware) that uses random } \\
\text { numbers or bits obtained from an approved random bit } \\
\text { generator. }\end{array}$ \\
\hline Cryptographic Key (Key) & $\begin{array}{l}\text { A parameter that determines the operation of a cryptographic } \\
\text { function, such as: } \\
\text { 1. The transformation from plaintext to ciphertext and } \\
\text { vice versa, }\end{array}$ \\
\hline
\end{tabular}




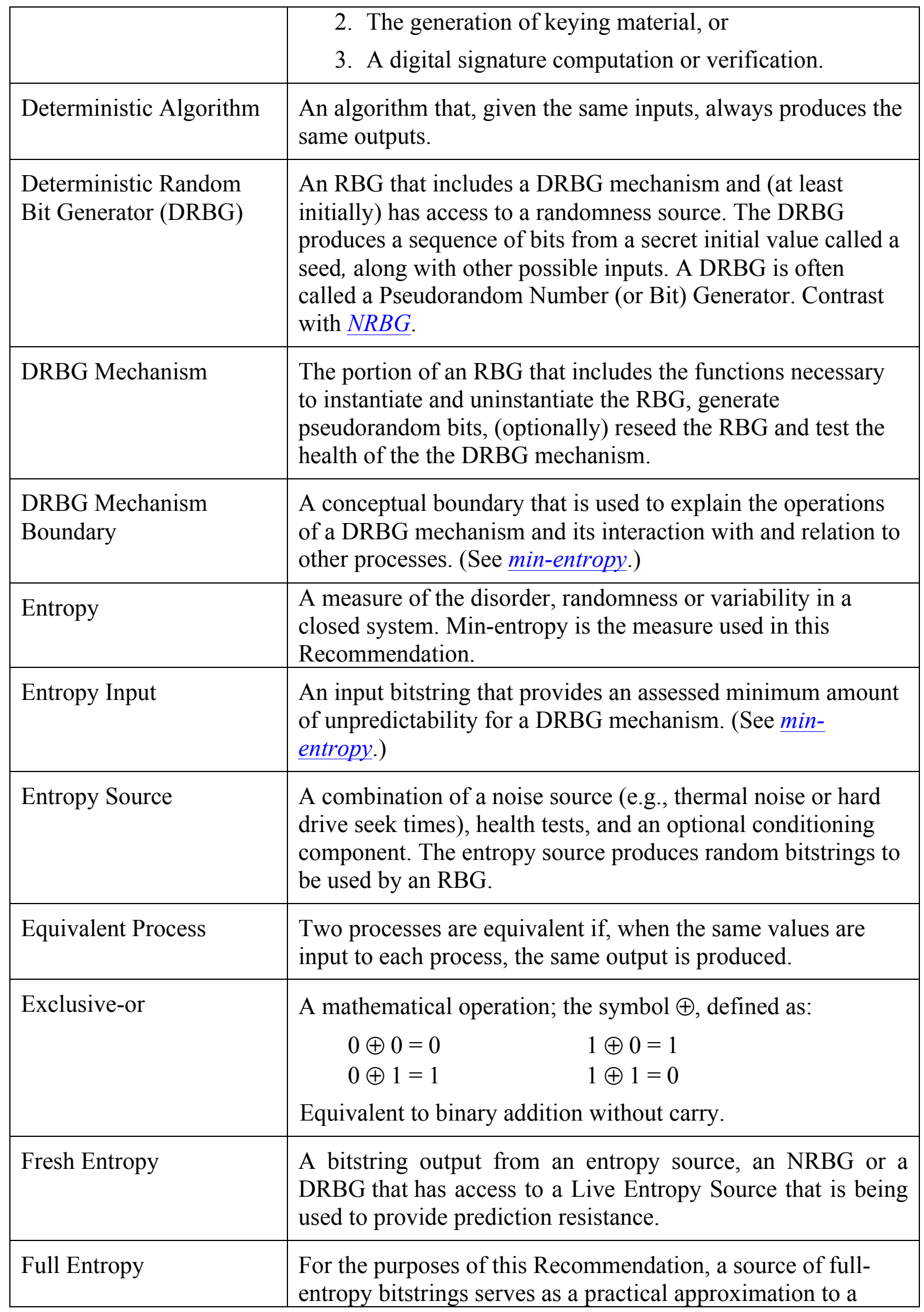




\begin{tabular}{|c|c|}
\hline & $\begin{array}{l}\text { source of ideal random bitstrings of the same length (see } \underline{i d e a l} \\
\text { random sequence). }\end{array}$ \\
\hline Hash Function & $\begin{array}{l}\text { A (mathematical) function that maps values from a large } \\
\text { (possibly very large) domain into a smaller range. The } \\
\text { function satisfies the following properties: } \\
\text { 1. (One-way) It is computationally infeasible to find any } \\
\text { input that maps to any pre-specified output; } \\
\text { 2. (Collision free) It is computationally infeasible to find } \\
\text { any two distinct inputs that map to the same output. }\end{array}$ \\
\hline Health Testing & $\begin{array}{l}\text { Testing within an implementation immediately prior to or } \\
\text { during normal operation to determine that the implementation } \\
\text { continues to perform as implemented and as validated. }\end{array}$ \\
\hline Ideal Random Bitstring & See Ideal Random Sequence. \\
\hline Ideal Random Sequence & $\begin{array}{l}\text { Each bit of an ideal random sequence is unpredictable and } \\
\text { unbiased, with a value that is independent of the values of the } \\
\text { other bits in the sequence. Prior to the observation of the } \\
\text { sequence, the value of each bit is equally likely to be } 0 \text { or } 1 \text {, } \\
\text { and the probability that a particular bit will have a particular } \\
\text { value is unaffected by knowledge of the values of any or all of } \\
\text { the other bits. An ideal random sequence of } n \text { bits contains } n \\
\text { bits of entropy. }\end{array}$ \\
\hline Implementation & $\begin{array}{l}\text { An implementation of an RBG is a cryptographic device or } \\
\text { portion of a cryptographic device that is the physical } \\
\text { embodiment of the RBG design, for example, some code } \\
\text { running on a computing platform. }\end{array}$ \\
\hline $\begin{array}{l}\text { Implementation Testing } \\
\text { for Validation }\end{array}$ & $\begin{array}{l}\text { Testing by an independent and accredited party to ensure that } \\
\text { an implementation of this Recommendation conforms to the } \\
\text { specifications of this Recommendation. }\end{array}$ \\
\hline Instantiation of an RBG & $\begin{array}{l}\text { An instantiation of an RBG is a specific, logically } \\
\text { independent, initialized RBG. One instantiation is } \\
\text { distinguished from another by a "handle" (e.g., an identifying } \\
\text { number). }\end{array}$ \\
\hline Internal State & $\begin{array}{l}\text { The collection of stored information about a DRBG } \\
\text { instantiation. This can include both secret and non-secret } \\
\text { information. Compare to working state. }\end{array}$ \\
\hline Key & See Cryptographic Key. \\
\hline Live Entropy Source & $\begin{array}{l}\text { An approved entropy source (see }[\mathrm{SP} 800-90 \mathrm{~B}] \text { ) that can } \\
\text { provide an } \mathrm{RBG} \text { with bits having a specified amount of }\end{array}$ \\
\hline
\end{tabular}




\begin{tabular}{|c|c|}
\hline & $\begin{array}{l}\text { entropy immediately upon request or within an acceptable } \\
\text { amount of time, as determined by the user or application } \\
\text { relying upon that RBG. }\end{array}$ \\
\hline Min-entropy & $\begin{array}{l}\text { The min-entropy (in bits) of a random variable } X \text { is the largest } \\
\text { value } m \text { having the property that each observation of } X \\
\text { provides at least } m \text { bits of information (i.e., the min-entropy of } \\
X \text { is the greatest lower bound for the information content of } \\
\text { potential observations of } X \text { ). The min-entropy of a random } \\
\text { variable is a lower bound on its entropy. The precise } \\
\text { formulation for min-entropy is - }\left(\log _{2} \text { max } p_{i}\right) \text { for a discrete } \\
\text { distribution having } n \text { possible outputs with probabilities } p_{1}, \ldots, \\
p_{n} \text {. Min-entropy is often used as a worst-case measure of the } \\
\text { unpredictability of a random variable. Also see [SP } 800-90 \mathrm{~B}] \text {. }\end{array}$ \\
\hline $\begin{array}{l}\text { Non-Deterministic } \\
\text { Random Bit Generator } \\
\text { (Non-deterministic RBG) } \\
\text { (NRBG) }\end{array}$ & $\begin{array}{l}\text { An RBG that always has access to an entropy source and } \\
\text { (when working properly) produces output bitstrings that have } \\
\text { full entropy. Often called a True Random Number (or Bit) } \\
\text { Generator. (Contrast with a deterministic random bit } \\
\text { generator). }\end{array}$ \\
\hline Nonce & $\begin{array}{l}\text { A time-varying value that has at most a negligible chance of } \\
\text { repeating, e.g., a random value that is generated anew for each } \\
\text { use, a timestamp, a sequence number, or some combination of } \\
\text { these. }\end{array}$ \\
\hline Personalization String & $\begin{array}{l}\text { An optional string of bits that is combined with a secret } \\
\text { entropy input and (possibly) a nonce to produce a seed. }\end{array}$ \\
\hline Prediction Resistance & $\begin{array}{l}\text { An RBG provides prediction resistance relative to time } T \text { if it } \\
\text { provides assurance that an adversary with knowledge of the } \\
\text { state of the RBG at some time(s) prior to } T \text { (but incapable of } \\
\text { performing work that matches the claimed security strength of } \\
\text { the RBG) would be unable to distinguish between } \\
\text { observations of ideal random bitstrings and (previously } \\
\text { unseen) bitstrings output by the RBG at or subsequent to time } \\
T \text {. In particular, an RBG whose design allows the adversary to } \\
\text { step forward from the initially compromised RBG state(s) to } \\
\text { obtain knowledge of subsequent RBG states and the } \\
\text { corresponding outputs (including the RBG state and output at } \\
\text { time } T \text { ) would not provide prediction resistance relative to } \\
\text { time } T \text {. (Contrast with backtracking resistance.) }\end{array}$ \\
\hline Pseudorandom & $\begin{array}{l}\text { A process (or data produced by a process) is said to be } \\
\text { pseudorandom when the outcome is deterministic, yet also } \\
\text { effectively random, as long as the internal action of the } \\
\text { process is hidden from observation. For cryptographic } \\
\text { purposes, "effectively" means "within the limits of the }\end{array}$ \\
\hline
\end{tabular}




\begin{tabular}{|c|c|}
\hline & intended cryptographic strength." \\
\hline $\begin{array}{l}\text { Pseudorandom Number } \\
\text { Generator }\end{array}$ & See Deterministic Random Bit Generator. \\
\hline Random Number & $\begin{array}{l}\text { For the purposes of this Recommendation, a value in a set that } \\
\text { has an equal probability of being selected from the total } \\
\text { population of possibilities and, hence, is unpredictable. A } \\
\text { random number is an instance of an unbiased random variable, } \\
\text { that is, the output produced by a uniformly distributed random } \\
\text { process. }\end{array}$ \\
\hline $\begin{array}{l}\text { Random Bit Generator } \\
\text { (RBG) }\end{array}$ & $\begin{array}{l}\text { A device or algorithm that outputs a sequence of binary bits } \\
\text { that appears to be statistically independent and unbiased. An } \\
\text { RBG is either a DRBG or an NRBG. }\end{array}$ \\
\hline Randomness Source & $\begin{array}{l}\text { A component of a DRBG (which consists of a DRBG } \\
\text { mechanism and a randomness source) that outputs bitstrings } \\
\text { that are used as entropy input by the DRBG mechanism. The } \\
\text { randomness source can be an entropy source or an RBG. }\end{array}$ \\
\hline Reseed & $\begin{array}{l}\text { To acquire additional bits that will affect the internal state of } \\
\text { the DRBG mechanism. }\end{array}$ \\
\hline Secure Channel & $\begin{array}{l}\text { A path for transferring data between two entities or } \\
\text { components that ensures confidentiality, integrity and replay } \\
\text { protection, as well as mutual authentication between the } \\
\text { entities or components. The secure channel may be provided } \\
\text { using approved cryptographic, physical or procedural } \\
\text { methods, or a combination thereof. Sometimes called a trusted } \\
\text { channel. }\end{array}$ \\
\hline Security Strength & $\begin{array}{l}\text { A number associated with the amount of work (that is, the } \\
\text { number of operations of some sort) that is required to break a } \\
\text { cryptographic algorithm or system in some way. In this } \\
\text { Recommendation, the security strength is specified in bits and } \\
\text { is a specific value from the set }\{112,128,192,256\} \text {. If the } \\
\text { security strength associated with an algorithm or system is } S \\
\text { bits, then it is expected that (roughly) } 2^{S} \text { basic operations are } \\
\text { required to break it. }\end{array}$ \\
\hline Seed & $\begin{array}{l}\text { Noun : A string of bits that is used as input to a DRBG } \\
\text { mechanism. The seed will determine a portion of the internal } \\
\text { state of the DRBG, and its entropy must be sufficient to } \\
\text { support the security strength of the DRBG. } \\
\text { Verb : To acquire bits with sufficient entropy for the desired } \\
\text { security strength. These bits will be used as input to a DRBG } \\
\text { mechanism to determine a portion of the initial internal state. }\end{array}$ \\
\hline
\end{tabular}




\begin{tabular}{|c|c|}
\hline & Also see reseed. \\
\hline Seedlife & The length of the seed period. \\
\hline Seed Period & $\begin{array}{l}\text { The period of time between instantiating or reseeding a DRBG } \\
\text { with one seed and reseeding that DRBG with another seed. }\end{array}$ \\
\hline Sequence & An ordered set of quantities. \\
\hline Shall & $\begin{array}{l}\text { Used to indicate a requirement of this Recommendation. } \\
\text { "Shall" may be coupled with "not" to become "shall not." }\end{array}$ \\
\hline Should & $\begin{array}{l}\text { Used to indicate a highly desirable feature for a DRBG } \\
\text { mechanism that is not necessarily required by this } \\
\text { Recommendation. "Should" may be coupled with "not" to } \\
\text { become "should not." }\end{array}$ \\
\hline Source of Randomness & See Randomness Source. \\
\hline String & See Bitstring. \\
\hline Unbiased & $\begin{array}{l}\text { A value that is chosen from a sample space is said to be } \\
\text { unbiased if all potential values have the same probability of } \\
\text { being chosen. Contrast with biased. }\end{array}$ \\
\hline Uninstantiate & The termination of a DRBG instantiation. \\
\hline Unpredictable & $\begin{array}{l}\text { In the context of random bit generation, an output bit is } \\
\text { unpredictable if an adversary has only a negligible advantage } \\
\text { (that is, essentially not much better than chance) in predicting } \\
\text { it correctly. }\end{array}$ \\
\hline Working State & $\begin{array}{l}\text { A subset of the internal state that is used by a DRBG } \\
\text { mechanism to produce pseudorandom bits at a given point in } \\
\text { time. The working state (and thus, the internal state) is } \\
\text { updated to the next state prior to producing another string of } \\
\text { pseudorandom bits. }\end{array}$ \\
\hline
\end{tabular}




\section{Symbols and Abbreviated Terms}

The following abbreviations are used in this Recommendation:

\begin{tabular}{|l|l|}
\hline Abbreviation & \multicolumn{1}{|c|}{ Meaning } \\
\hline AES & Advanced Encryption Standard, as specified in [FIPS 197]. \\
\hline DRBG & Deterministic Random Bit Generator. \\
\hline FIPS & Federal Information Processing Standard. \\
\hline HMAC & Keyed-Hash Message Authentication Code, as specified in [FIPS 198]. \\
\hline NIST & National Institute of Standards and Technology. \\
\hline NRBG & Non-deterministic Random Bit Generator. \\
\hline RBG & Random Bit Generator. \\
\hline SP & NIST Special Publication. \\
\hline TDEA & Triple Data Encryption Algorithm, as specified in [SP 800-67]. \\
\hline
\end{tabular}

The following symbols are used in this Recommendation:

\begin{tabular}{|l|l|}
\hline \multicolumn{1}{|c|}{ Symbol } & \\
\hline+ & Addition. \\
\hline$X \oplus Y$ & $\begin{array}{l}\text { Bitwise exclusive-or (also bitwise addition modulo 2) of two bitstrings } \\
X \text { and } Y \text { of the same length. }\end{array}$ \\
\hline$X \| Y$ & $\begin{array}{l}\text { Concatenation of two strings } X \text { and } Y . X \text { and } Y \text { are either both bitstrings } \\
\text { or both byte strings. }\end{array}$ \\
\hline$\lceil x\rceil$ & $\begin{array}{l}\text { The ceiling of } x ; \text { the smallest integer } \geq x . \text { For example, }\lceil 5\rceil=5, \text { and } \\
\lceil 5.3\rceil=6 .\end{array}$ \\
\hline leftmost $(V, a)$ & The leftmost $a$ bits of $V$. \\
\hline len $(a)$ & The length in bits of string $a$. \\
\hline min $(a, b)$ & The minimum of $a$ and $b$. \\
\hline$x$ mod $n$ & $\begin{array}{l}\text { The unique remainder } r(\text { where } 0 \leq r \leq n-1) \text { when integer } x \text { is divided } \\
\text { by } n . \text { For example, } 23 \text { mod } 7=2 .\end{array}$ \\
\hline rightmost $(V, a)$ & The rightmost $a$ bits of $V$. \\
\hline select $(V, a, b)$ & A substring of string $V$ consisting of bit $a$ through bit $b$. \\
\hline$\left\{\begin{array}{l}\text { Thed in a figure to illustrate a "switch" between input sources. } \\
\hline\end{array}\right.$ & $\begin{array}{l}\text { The internal state of the DRBG at a point in time. The types and } \\
\text { number of the } a_{i} \text { values depends on the specific DRBG mechanism. }\end{array}$ \\
\hline
\end{tabular}




\begin{tabular}{|l|l|}
\hline \multicolumn{1}{|c|}{ Symbol } & \multicolumn{1}{c|}{ Meaning } \\
\hline $0 \mathrm{x} a b$ & $\begin{array}{l}\text { Hexadecimal notation that is used to define a byte (i.e., eight bits) of } \\
\text { information, where } a \text { and } b \text { each specify four bits of information and } \\
\text { have values from the range }\{0,1,2, \ldots \mathrm{F}\} . \text { For example, } 0 \mathrm{xc} 6 \text { is used to } \\
\text { represent } 11000110, \text { where } \mathrm{c} \text { is } 1100, \text { and } 6 \text { is } 0110 .\end{array}$ \\
\hline $0^{x}$ & A string of $x$ zero bits. \\
\hline
\end{tabular}

\section{Document Organization}

This Recommendation is organized as follows:

- Section 7 provides a functional model for a DRBG that uses a DRBG mechanism and discusses the major components of the DRBG mechanism.

- Section 8 provides concepts and general requirements for the implementation and use of a DRBG mechanism.

- Section 9 specifies the functions of a DRBG mechanism that were introduced in Section 8. These functions use the DRBG algorithms specified in Section 10.

- Section 10 specifies approved DRBG algorithms. Algorithms have been specified that are based on the hash functions specified in [FIPS 180], and the block cipher algorithms specified in [FIPS 197] and [SP 800-67] (AES and TDEA, respectively).

- Section 11 addresses assurance issues for DRBG mechanisms, including documentation requirements, and implementation validation and health testing.

This Recommendation also includes the following appendices:

- Appendix A provides conversion routines.

- Appendix B provides example pseudocode for each DRBG mechanism. Examples of the values computed for the DRBGs using each approved cryptographic algorithm and key size are available at http://csrc.nist.gov/groups/ST/toolkit/examples.html under the entries for SP 800-90A.

- Appendix C provides a discussion on DRBG mechanism selection.

- Appendix D provides references.

- Appendix E provides a list of modifications to SP 800-90A since it was first published. 


\section{Functional Model of a DRBG}

Figure 1 provides a functional model of a DRBG (i.e., one type of RBG). A DRBG shall implement an approved DRBG mechanism from SP 800-90A and at least one approved randomness source (see Section 8.6.5), and may include additional optional sources, including sources for nonces, personalization strings, and additional input. The components of this model are discussed in the following subsections. DRBG constructions are also discussed in [SP 80090C].

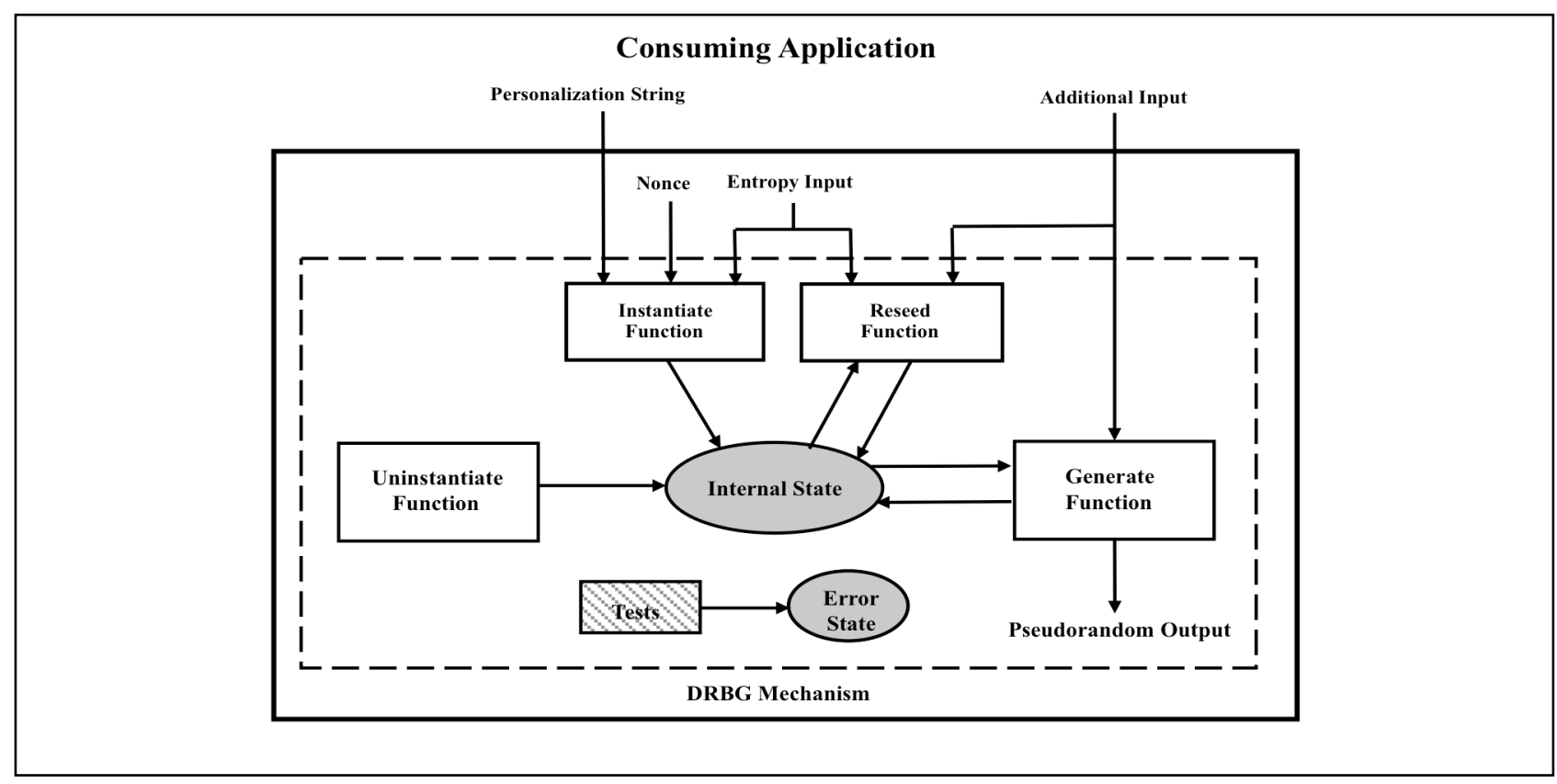

Figure 1: DRBG Functional Model

\subsection{Entropy Input}

Entropy input is provided to a DRBG mechanism for the seed (see Section 8.6) using a randomness source. The entropy input and the seed shall be kept secret. The secrecy of this information provides the basis for the security of the DRBG. At a minimum, the randomness source shall provide input that supports the security strength requested by the DRBG mechanism. Appropriate randomness sources are discussed in Section 8.6.5.

Ideally, the entropy input would have full entropy; however, the DRBG mechanisms have been specified so that input with full entropy is not required. This is accommodated by allowing the length of the entropy input to be longer than the required entropy (expressed in bits), as long as the total entropy meets the requirements of the DRBG mechanism. The entropy input can be defined to be of variable length (within specified limits), as well as fixed length. In all cases, the DRBG mechanism expects that when entropy input is requested, the returned bitstring will contain at least the requested amount of entropy. Additional entropy beyond the amount requested is not required, but is desirable. 


\subsection{Other Inputs}

Other information may be obtained by a DRBG mechanism as input. This information may or may not be required to be kept secret by a consuming application; however, the security of the DRBG itself does not rely on the secrecy of this information. The information should be checked for validity when possible; for example, if time is used as an input, the format and reasonableness of the time could be checked. In most cases, a nonce is required during instantiation (see Sections 8.6.1 and 8.6.7). When required, the nonce is combined with the entropy input to create the initial DRBG seed.

A personalization string should be used during DRBG instantiation; when used, the personalization string is combined with the entropy input bits and possibly a nonce to create the initial DRBG seed. The personalization string should be unique for all instantiations of the same DRBG mechanism type (e.g., all instantiations of HMAC_DRBG). See Section 8.7.1 for additional discussion on personalization strings.

Additional input may also be provided during reseeding and when pseudorandom bits are requested. See Section 8.7.2 for a discussion of this input.

\subsection{The Internal State}

The internal state is the memory of the DRBG and consists of all of the parameters, variables and other stored values that the DRBG mechanism uses or acts upon. The internal state contains both administrative data (e.g., the security strength) and data that is acted upon and/or modified during the generation of pseudorandom bits (i.e., the working state).

\subsection{The DRBG Mechanism Functions}

The DRBG mechanism functions handle the DRBG's internal state. The DRBG mechanisms in this Recommendation have five separate functions:

1. The instantiate function acquires entropy input and may combine it with a nonce and a personalization string to create a seed from which the initial internal state is created.

2. The generate function generates pseudorandom bits upon request, using the current internal state and possibly additional input; a new internal state for the next request is also generated.

3. The reseed function acquires new entropy input and combines it with the current internal state and any additional input that is provided to create a new seed and a new internal state.

4. The uninstantiate function zeroizes (i.e., erases) the internal state.

5. The health test function determines that the DRBG mechanism continues to function correctly. 


\section{DRBG Mechanism Concepts and General Requirements}

\subsection{DRBG Mechanism Functions}

A DRBG mechanism requires instantiate, uninstantiate, generate, and health testing functions. A DRBG mechanism includes an optional reseed function. A DRBG shall be instantiated prior to the generation of output by the DRBG. These functions are specified in Section 9.

\subsection{DRBG Instantiations}

A DRBG may be used to obtain pseudorandom bits for different purposes (e.g., DSA private keys and AES keys) and may be separately instantiated for each purpose, thus effectively creating two DRBGs.

A DRBG is instantiated using a seed and may be reseeded; when reseeded, the seed shall be different than the seed used for instantiation. Each seed defines a seed period for the DRBG instantiation; an instantiation consists of one or more seed periods that begin when a new seed is

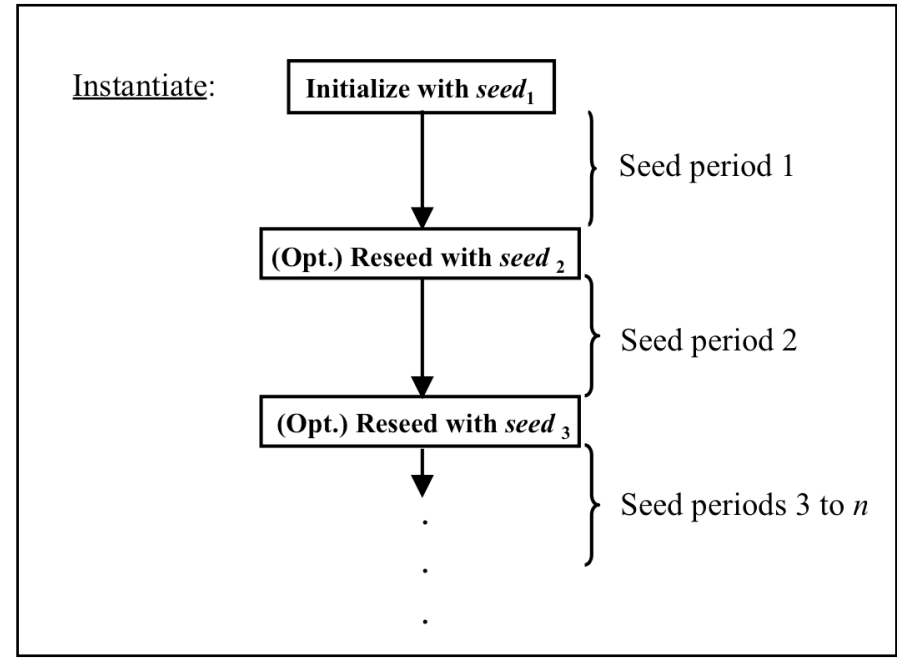

Figure 2: DRBG Instantiation acquired and end when the next seed is obtained or the DRBG is no longer used (see Figure 2).

\subsection{Internal States}

During instantiation, an initial internal state is derived from the seed. The internal state for an instantiation includes:

1. The working state:

a. One or more values that are derived from the seed and become part of the internal state; these values shall remain secret, and

b. A count of the number of requests produced since the instantiation was seeded or reseeded.

2. Administrative information (e.g., security strength and prediction resistance flag).

The internal state shall be protected at least as well as the intended use of the pseudorandom output bits requested by the consuming application. A DRBG mechanism implementation may be designed to handle multiple instantiations. Each DRBG instantiation shall have its own internal state. The internal state for one DRBG instantiation shall not be used as the internal state for a different instantiation. 


\subsection{Security Strengths Supported by an Instantiation}

The DRBG mechanisms specified in this Recommendation support four security strengths: 112, 128, 192 or 256 bits. The security strength for the instantiation is requested during DRBG instantiation, and the instantiate function obtains the appropriate amount of entropy for the requested security strength. Each DRBG mechanism has restrictions on the security strength it can support, based on its design (see Section 10).

The actual security strength supported by a given instantiation depends on the DRBG implementation and on the amount of entropy provided to the instantiate function. Note that the security strength actually supported by a particular instantiation could be less than the maximum security strength possible for that DRBG implementation (see Table 1). For example, a DRBG that is designed to support a maximum security strength of 256 bits could, instead, be instantiated to support only a 128-bit security strength if the additional security provided by the 256-bit security strength is not required (e.g., by requesting only 128 bits of entropy during instantiation, rather than 256 bits of entropy).

Table 1: Possible Instantiated Security Strengths

\begin{tabular}{|c|c|c|c|c|}
\hline $\begin{array}{c}\text { Maximum Designed } \\
\text { Security Strength }\end{array}$ & $\mathbf{1 1 2}$ & $\mathbf{1 2 8}$ & $\mathbf{1 9 2}$ & $\mathbf{2 5 6}$ \\
\hline $\begin{array}{c}\text { Possible Instantiated } \\
\text { Security Strengths }\end{array}$ & 112 & 112,128 & $112,128,192$ & $\begin{array}{c}112,128,192, \\
256\end{array}$ \\
\hline
\end{tabular}

Following instantiation, a request can be made to the generate function for pseudorandom bits (see Section 9.3). The pseudorandom bits returned from a DRBG shall not be used for any application that requires a higher security strength than the DRBG is instantiated to support. The security strength provided in these returned bits is the minimum of the security strength supported by the DRBG and the length of the bit string returned, i.e.:

$$
\text { Security_strength_of_output }=\min (\text { output_length, DRBG_security_strength) } \text {. }
$$

A concatenation of bit strings resulting from multiple calls to a DRBG will not provide a security strength for the concatenated string that is greater than the instantiated security strength of the DRBG. For example, two 128-bit output strings requested from a DRBG that supports a128-bit security strength cannot be concatenated to form a 256-bit string with a security strength of 256 bits. A more complete discussion of this issue is provided in [SP 800-90C].

For each generate request, the security strength to be provided for the bits is requested. Any security strength can be requested during a call to the generate function, up to the security strength of the instantiation, e.g., an instantiation could be instantiated at the 128-bit security strength, but a request for pseudorandom bits could indicate that a lesser security strength is actually required for the bits to be generated. Assuming that the request is valid, the requested number of bits is returned.

When an instantiation is used for multiple purposes, the minimum security strength requirement for each purpose must be considered. The DRBG needs to be instantiated for the highest security strength required. For example, if one purpose requires a security strength of 112 bits, and 
another purpose requires a security strength of 256 bits, then the DRBG needs to be instantiated to support the 256-bit security strength.

\subsection{DRBG Mechanism Boundaries}

As a convenience, this Recommendation uses the notion of a "DRBG mechanism boundary" to explain the operations of a DRBG mechanism and its interaction with and relation to other processes; a DRBG mechanism boundary contains all DRBG mechanism functions and internal states required for a DRBG. Data enters a DRBG mechanism boundary via the DRBG's public interfaces, which are made available to consuming applications.

The DRBG mechanism boundary should not be confused with a cryptographic module boundary, as specified in [FIPS 140]; the relationship between a cryptographic module boundary and a DRBG boundary is mentioned below, but is more fully discussed in [SP 800-90C].

Within a DRBG mechanism boundary,

1. The DRBG internal state and the operation of the DRBG mechanism functions shall only be affected according to the DRBG mechanism specification.

2. The DRBG internal state shall exist solely within the DRBG mechanism boundary. The internal state shall not be accessible by non-DRBG functions or other instantiations of that DRBG or other DRBGs.

3. Information about secret parts of the DRBG internal state and intermediate values in computations involving these secret parts shall not affect any information that leaves the DRBG mechanism boundary, except as specified for the DRBG pseudorandom bit outputs.

Each DRBG mechanism includes one or more cryptographic primitives (i.e., a hash function or block cipher algorithm). Other applications may use the same cryptographic primitive, but the DRBG's internal state and the DRBG mechanism functions shall not be affected by these other applications. For example, a DRBG mechanism may use the same hash-function code as a digital-signature application.

A DRBG mechanism's functions may be contained within a single device, or may be distributed across multiple devices (see Figures 3 and 4). Figure 3 depicts a DRBG for which all functions are contained within the same device. As further discussed in [SP 800-90C], the DRBG mechanism boundary (in this case) is contained within a cryptographic module boundary. 


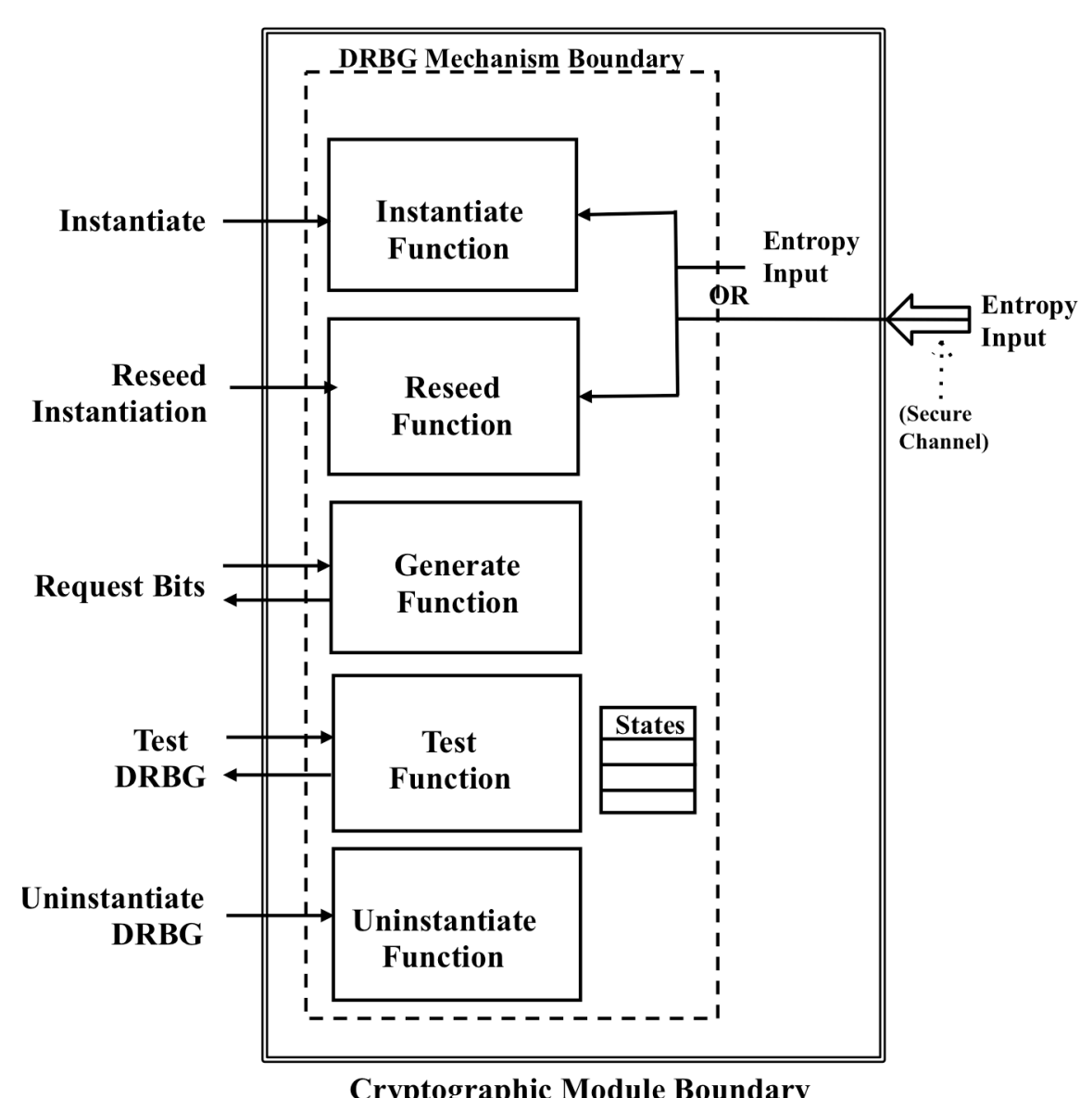

Figure 3: DRBG Mechanism Functions within a Single Device

Figure 4 provides an example of DRBG mechanism functions that are distributed across multiple devices. In this case, each device has a DRBG mechanism sub-boundary that contains the DRBG mechanism functions implemented on that device, and the DRBG mechanism sub-boundary is contained within a cryptographic module boundary, as is further discussed in [SP 800-90C]. The boundary around the entire DRBG mechanism includes the aggregation of sub-boundaries providing the DRBG mechanism functionality. Each sub-boundary may be contained within a different cryptographic module boundary, or multiple sub-boundaries may be contained within the same cryptographic module boundary.

The use of distributed DRBG-mechanism functions may be convenient for restricted environments (e.g., smart card applications) in which the primary use of the DRBG does not require repeated use of the instantiate or reseed functions.

Each DRBG mechanism boundary or sub-boundary shall contain a health test function to test the "health" of other DRBG-mechanism functions within that boundary. In addition, a boundary or sub-boundary that contains an instantiate function shall contain an uninstantiate function in order to terminate an instantiation. 


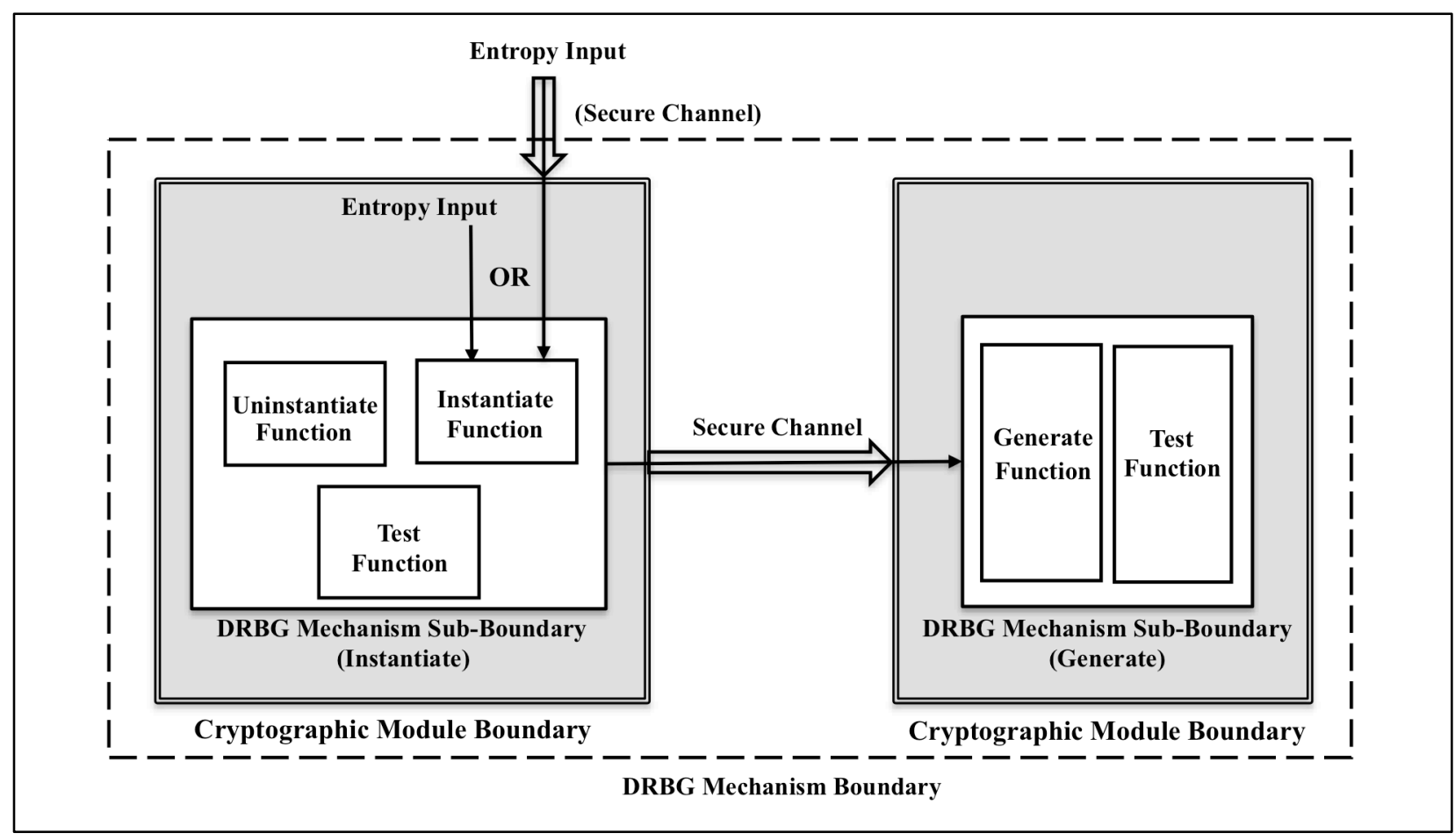

Figure 4: Distributed DRBG Mechanism Functions

When DRBG mechanism functions are distributed, a physically or cryptographically secure channel shall be used to protect the confidentiality and integrity of the internal state or parts of the internal state that are transferred between the distributed DRBG mechanism sub-boundaries. The security provided by the secure channel shall be consistent with the security required by the consuming application. See Section 4 for a more complete definition of a secure channel.

For distributed DRBGs, each sub-boundary is the same as or is fully contained within a cryptographic module boundary.

\subsection{Seeds}

When a DRBG is used to generate pseudorandom bits, a seed shall be acquired prior to the generation of output bits by the DRBG. The seed is used to instantiate the DRBG and determine the initial internal state that is used when calling the DRBG to obtain the first output bits.

Reseeding is a means of restoring the secrecy of the output of the DRBG if a seed or the internal state becomes known. Periodic reseeding is a good way of addressing the threat of either the DRBG seed, entropy input or working state being compromised over time. In some implementations (e.g., smartcards), an adequate reseeding process may not be possible. In these cases, the best policy might be to replace the DRBG, obtaining a new seed in the process (e.g., obtain a new smart card).

The seed and its use by a DRBG mechanism shall be generated and handled as specified in the following subsections. 


\subsubsection{Seed Construction for Instantiation}

Figure 5 depicts the seed-construction process for instantiation. The seed material used to determine a seed for instantiation consists of entropy input from a randomness source, a nonce and an optional (but recommended) personalization string. Entropy input shall always be used in the construction of a seed; requirements for the entropy input are discussed in Section 8.6.3. Except for the case noted below, a nonce shall be used; requirements for the nonce are discussed in Section 8.6.7. A personalization string should also be used;

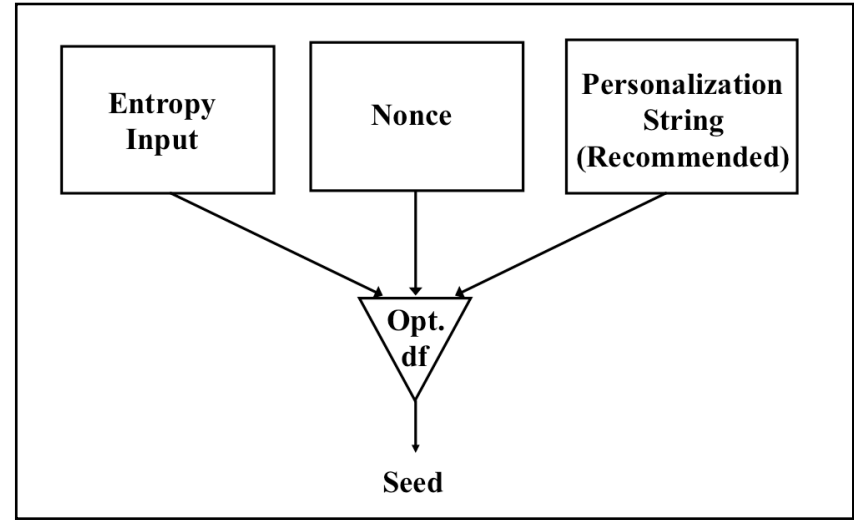

Figure 5: Seed Construction for Instantiation requirements for the personalization string are discussed in Section 8.7.1.

Depending on the DRBG mechanism and the randomness source, a derivation function may be required to derive a seed from the seed material. However, in certain circumstances, the CTR_DRBG mechanism based on block cipher algorithms (see Section 10.2) may be implemented without a derivation function. When implemented in this manner, a (separate) nonce (as shown in Figure 5) is not used. Note, however, that the personalization string could contain a nonce, if desired.

\subsubsection{Seed Construction for Reseeding}

Figure 6 depicts the seed construction process for reseeding an instantiation. The seed material for reseeding consists of a value that is carried in the internal state ${ }^{3}$, new entropy input and, optionally, additional input. The internal state value and the entropy input are required; requirements for the entropy input are discussed in Section 8.6.3. Requirements for the additional input are discussed in Section

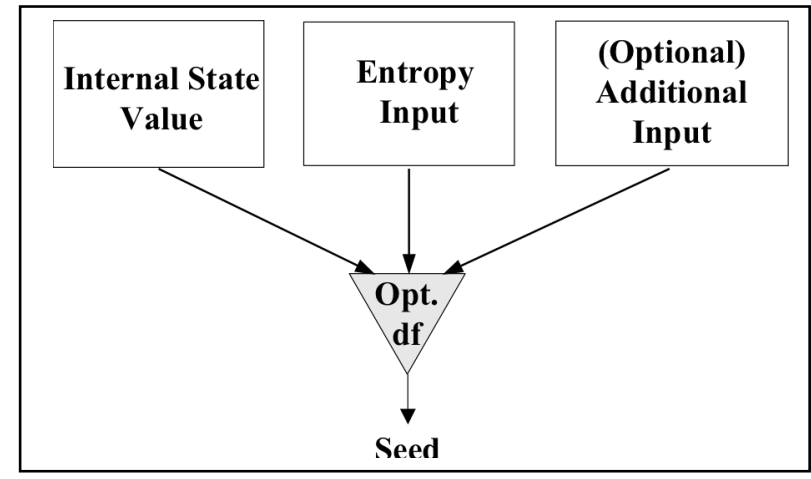

Figure 6: Seed Construction for Reseeding 8.7.2. As in Section 8.6.1, a derivation function may be required for reseeding.

\subsubsection{Entropy Requirements for the Entropy Input}

The entropy input shall have entropy that is equal to or greater than the security strength of the instantiation. Additional entropy may be provided in the nonce or the optional personalization string during instantiation, or in the additional input during reseeding and generation, but this is not required and does not increase the "official" security strength of the DRBG instantiation that is recorded in the internal state. The use of more entropy than the minimum value will offer a

\footnotetext{
${ }^{3}$ See each DRBG mechanism specification for the value that is used.
} 
security "cushion". This may be useful if the assessment of the entropy provided in the entropy input is incorrect. Having more entropy than the assessed amount is acceptable; having less entropy than the assessed amount could be fatal to security. The presence of more entropy than is required, especially during the instantiation, will provide a higher level of assurance than the minimum required entropy.

\subsubsection{Seed Length}

The minimum length of the seed depends on the DRBG mechanism and the security strength required by the consuming application, but shall be at least the number of bits of entropy required. See the tables in Section 10.

\subsubsection{Randomness Source}

A DRBG mechanism requires an approved randomness source during instantiation and reseeding, including whenever prediction resistance is requested (see Section 8.8). This input is requested using the Get_entropy_input function introduced in Section 9 and is specified in more detail in [SP 800-90C].

An approved randomness source is an entropy source that conforms to [SP 800-90B], or an RBG that conforms to [SP 800-90C] - either a DRBG or an NRBG.

\subsubsection{Entropy Input and Seed Privacy}

The entropy input and the resulting seed shall be handled in a manner that is consistent with the security required for the data protected by the consuming application. For example, if the DRBG is used to generate keys, then the entropy inputs and seeds used to generate the keys shall (at a minimum) be protected at the same security strength as the keys.

The security of the DRBG depends on the secrecy of the entropy input. For this reason, the entropy input shall be treated as a critical security parameter (CSP) during cryptographic module validation. The entropy input for the DRBG function requiring the entropy input shall be obtained either from within the cryptographic module containing that function or from another cryptographic module and transported to the DRBG function's cryptographic module via a secure channel.

\subsubsection{Nonce}

A nonce may be required in the construction of a seed during instantiation in order to provide a security cushion to block certain attacks. The nonce shall be either:

a. A value with at least (security_strength/2) bits of entropy, or

b. A value that is expected to repeat no more often than a (security_strength/2)-bit random string would be expected to repeat.

Each nonce shall be unique to the cryptographic module in which instantiation is performed, but need not be secret. When used, the nonce shall be considered to be a critical security parameter.

A nonce may be composed of one (or more) of the following components (other components may also be appropriate): 
1. A random value that is generated anew for each nonce, using an approved random bit generator.

2. A timestamp of sufficient resolution (detail) so that it is different each time it is used.

3. A monotonically increasing sequence number, or

4. A combination of a timestamp and a monotonically increasing sequence number, such that the sequence number is reset when and only when the timestamp changes. For example, a timestamp may show the date but not the time of day, so a sequence number is appended that will not repeat during a particular day.

For case 1 above, the random value could be acquired from the same source and at the same time as the entropy input. In this case, the seed could be considered to be constructed from an "extra strong" entropy input and the optional personalization string, where the entropy for the entropy input is equal to or greater than (3/2 security_strength) bits.

For case 2 above, the timestamp must be trusted. A trusted timestamp is generated and signed by an entity that is trusted to provide accurate time information.

The nonce provides greater assurance that the DRBG provides security_strength bits of security to the consuming application. If a DRBG were instantiated many times without a nonce, a compromise could become more likely. In some consuming applications, a single DRBG compromise could reveal long-term secrets (e.g., a compromise of the DSA per-message secret could reveal the signing key).

A nonce shall be generated within a cryptographic module boundary. This requirement does not preclude the generation of the nonce within a cryptographic module that is different from the cryptographic boundary containing the DRBG function with which the nonce is used (e.g., the cryptographic module boundary containing an instantiate function). However, in this scenario, there needs to be a secure channel to transport the nonce between the cryptographic-module boundaries. See the discussion of distributed DRBGs in Section 8.5 and distributed RBGs in [SP 800-90C].

\subsubsection{Reseeding}

Generating too many outputs from a seed (and other input information) may provide sufficient information for successfully predicting future outputs (see Section 8.8). Periodic reseeding will reduce security risks, reducing the likelihood of a compromise of the data that is protected by cryptographic mechanisms that use the DRBG.

Seeds have a finite seedlife (i.e., the number of outputs that are produced during a seed period); the maximum seedlife is dependent on the DRBG mechanism used. Implementations shall enforce the limits on seedlife specified for the DRBG mechanism used or more stringent limits selected by the implementer. When a DRBG's maximum seedlife is reached, the DRBG shall not generate outputs until it has been reseeded.

Reseeding is accomplished 1) by an explicit reseeding of the DRBG by the consuming application, 2) by the generate function when prediction resistance is requested (see Section 8.8) or 3) when the end of the seed life is determined during the generate function (see Section 9.3.1).

The reseeding of the DRBG shall be performed in accordance with the specification for a given DRBG mechanism. The DRBG reseed specifications within this Recommendation are designed 
to produce a new seed that is determined by both the old seed and newly obtained entropy input that will support the desired security strength.

An alternative to reseeding would be to create an entirely new instantiation. However, reseeding is preferred over creating a new instantiation. If a DRBG instantiation was initially seeded with sufficient entropy, and the randomness source subsequently fails without being detected, then a new instantiation using the same (failed) source would not have sufficient entropy to operate securely. However, if there is an undetected failure in the randomness source of an already properly seeded DRBG instantiation, the DRBG instantiation will still retain any previous entropy when the reseed operation fails to introduce new entropy.

\subsubsection{Seed Use}

The seed that is used to initialize one instantiation of a DRBG shall not be intentionally used to reseed the same instantiation or used as the seed for another DRBG instantiation. In addition, a DRBG instantiation shall not reseed itself. Note that a DRBG does not provide output until a seed is available, and the internal state has been initialized (see Section 10).

\subsubsection{Entropy Input and Seed Separation}

The seed used by a DRBG and the entropy input used to create that seed shall not intentionally be used for other purposes (e.g., domain parameter or prime number generation).

\subsection{Other Input to the DRBG Mechanism}

Other input may be provided during DRBG instantiation, generation and reseeding. This input may contain entropy, but this is not required. During instantiation, a personalization string may be provided and combined with entropy input and a nonce to derive a seed (see Section 8.6.1). When pseudorandom bits are requested and when reseeding is performed, additional input may be provided (see Section 8.7.2).

Depending on the method for acquiring the input, the exact value of the input may or may not be known to the user or consuming application. For example, the input could be derived directly from values entered by the user or consuming application, or the input could be derived from information introduced by the user or consuming application (e.g., from timing statistics based on key strokes), or the input could be the output of another RBG.

\subsubsection{Personalization String}

A personalization string is an optional (but recommended) input to the instantiate function and is used to derive the seed (see Section 8.6.1). The personalization string may be obtained from inside or outside a cryptographic module, and may be an empty string. Note that a DRB does not rely on a personalization string to provide entropy, even though entropy could be provided in the personalization string, and knowledge of the personalization string by an adversary does not degrade the security strength of a DRBG instantiation, as long as the entropy input is unknown. When used within a cryptographic module, a personalization string is not considered to be a critical security parameter. 
The personalization string may contain secret information, but shall not include secret information that requires protection at a higher security strength than the DRBG being instantiated will support. For example, a personalization string to be used to instantiate a DRBG at 112 bits of security strength shall not include information requiring 128 bits of protection. A given implementation of a DRBG may support the use of a personalization string, but is not required to do so.

The intent of a personalization string is to introduce additional input into the instantiation of a DRBG. This personalization string might contain values unknown to an attacker, or values that tend to differentiate this DRBG instantiation from all others. Ideally, a personalization string will be set to some bitstring that is as unique as possible. Good sources for the personalization string contents include:

- Application identifiers,

- Device serial numbers,

- User identification,

- Per-module or per-device values,

- Timestamps,

- Network addresses,
- Special key values for this specific DRBG instantiation,

- Protocol version identifiers,

- Random numbers,

- Nonces, and

- Outputs from other approved or nonapproved random bit generators.

\subsubsection{Additional Input}

Additional input may optionally be provided to the reseed and generate functions during requests. The additional input may be obtained from inside or outside a cryptographic module, and may include secret or public information. Note that a DRBG does not rely on additional input to provide entropy, even though entropy could be provided in the additional input, and knowledge of the additional input by an adversary does not degrade the security strength of a DRBG. However, if the additional input contains secret/private information (e.g., a social security number), that information shall not require protection at a higher security strength than the security strength supported by the DRBG. A given implementation of a DRBG may include the additional input, but is not required to do so. When used within a cryptographic module, the additional input used in DRBG requests is not considered to be a critical security parameter unless any secret information included in the additional input qualifies as a critical security parameter.

Additional input is optional for both the DRBG and the consuming application, and the ability to enter additional input may or may not be included in an implementation. The value of the additional input may be either secret or publicly known; its value is arbitrary, although its length may be restricted, depending on the implementation and the DRBG mechanism. The use of additional input may be a means of providing more entropy for the DRBG internal state that will increase assurance that the entropy requirements are met. If the additional input is kept secret and has sufficient entropy, the input can provide more assurance when recovering from the compromise of the entropy input, the seed or one or more DRBG internal states. 


\subsection{Prediction Resistance and Backtracking Resistance}

Figure 7 depicts the sequence of DRBG internal states that result from a given seed. Some subset of bits from each internal state are used to generate pseudorandom bits upon request by a user. The following discussions will use the figure to explain backtracking and prediction resistance.

Suppose that a compromise occurs at State $_{x}$, where State $_{x}$ contains both secret and non-secret information.

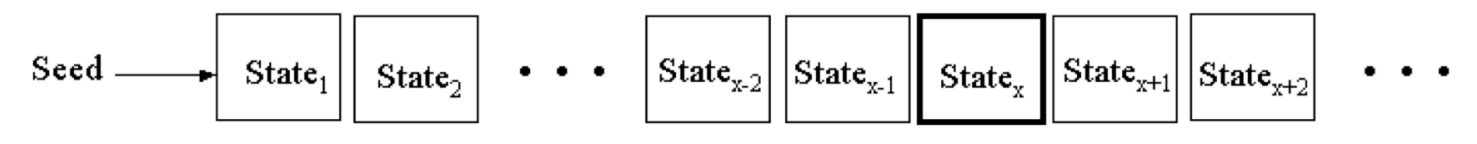

Figure 7: Sequence of DRBG States

Backtracking Resistance: Backtracking resistance is provided relative to time $T$ if there is assurance that an adversary who has knowledge of the internal state of the DRBG at some time subsequent to time $T$ would be unable to distinguish between observations of ideal random bitstrings and (previously unseen) bitstrings that were output by the DRBG prior to time $T$. This assumes that the adversary is incapable of performing the work required to negate the claimed security strength of the DRBG. Backtracking resistance means that a compromise of the DRBG internal state has no effect on the security of prior outputs. That is, an adversary who is given access to all of the prior output sequence cannot distinguish it from random output with less work than is associated with the security strength of the instantiation; if the adversary knows only part of the prior output, he cannot determine any bit of that prior output sequence that he has not already seen with better than a 50-50 chance.

For example, suppose that an adversary knows State $_{x}$. Backtracking resistance means that:

a. The output bits from State $_{1}$ to State $_{x-1}$ cannot be distinguished from random output, and

b. The prior internal state values themselves $\left(\right.$ State $_{1}$ to State $\left._{x-1}\right)$ cannot be recovered, given knowledge of the secret information in State $_{x}$.

Backtracking resistance can be provided by ensuring that the DRBG generation algorithm is a one-way function. All DRBG mechanisms in this Recommendation have been designed to provide backtracking resistance.

Prediction Resistance: Prediction resistance means that a compromise of the DRBG internal state has no effect on the security of future DRBG outputs. That is, an adversary who is given access to all of the output sequence after the compromise cannot distinguish it from random output with less work than is associated with the security strength of the instantiation; if the adversary knows only part of the future output sequence, he cannot predict any bit of that future output sequence that he does not already know (with better than a 50-50 chance).

For example, suppose that an adversary knows State $_{x}$. Prediction resistance means that:

a. The output bits from State $_{x+1}$ and forward cannot be distinguished from an ideal random bitstring by the adversary, and 
b. The future internal state values themselves (State St $_{1}$ and forward) cannot be predicted (with better than a 50-50 chance), given knowledge of State .

Prediction resistance is provided relative to time $T$ if there is assurance that an adversary with knowledge of the state of the RBG at some time(s) prior to $T$ (but incapable of performing work that matches the claimed security strength of the RBG) would be unable to distinguish between observations of ideal random bitstrings and (previously unseen) bitstrings output by the RBG at or subsequent to time $T$. In particular, an RBG whose design allows the adversary to step forward from the initially compromised RBG state(s) to obtain knowledge of subsequent RBG states and the corresponding outputs (including the RBG state and output at time $T$ ) would not provide prediction resistance relative to time $T$.

Prediction resistance can be provided only by ensuring that a DRBG is effectively reseeded with fresh entropy between producing output for consecutive DRBG requests. That is, an amount of entropy that is sufficient to support the security strength of the DRBG being reseeded (i.e., an amount that is at least equal to the security strength) must be provided to the DRBG in a way that ensures that knowledge of the current DRBG internal state does not allow an adversary any useful knowledge about future DRBG internal states or outputs. Prediction resistance can be provided when the randomness source is or has direct or indirect access to an entropy source or an NRBG (see Section 8.6.5).

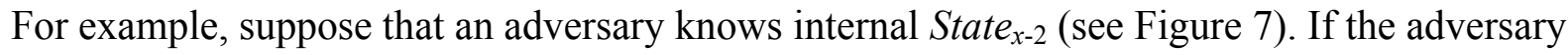
also knows the DRBG mechanism used, he then has enough information to compute State $_{x-1}$ and State $_{x}$. If prediction is then requested for the next bits that are to be output from the DRBG, new entropy bits will be inserted into the DRBG instantiation before State $_{x+1}$ is produced that will create a separation between State $_{x}$ and State $_{x+1}$, i.e., the adversary will not be able to compute State $_{x+1}$, simply by knowing State $_{x}$; the work required will be greatly increased by the entropy inserted during the prediction request.

The introduction of fresh entropy via reseeding will also make the DRBG less susceptible to cryptanalytic attack. Whenever an entropy source is available, it is strongly recommended that DRBGs be requested to provide prediction resistance as often as is practical. 


\section{DRBG Mechanism Functions}

All DRBG mechanisms and algorithms are described in this document in pseudocode, which is intended to explain functionality. The pseudocode is not intended to constrain real-world implementations.

Except for the health test function, which is discussed in Section 11.3, the functions of the DRBG mechanisms in this Recommendation are specified as an algorithm and an "envelope" of pseudocode around that algorithm. The pseudocode in each envelope (provided in this section) checks the input parameters, obtains input not provided via the input parameters, accesses the appropriate DRBG algorithm and manages the internal state. A function need not be implemented using such envelopes, but the function shall have equivalent functionality.

During instantiation and reseeding (see Sections 9.1 and 9.2), entropy input and (usually) a nonce are acquired for constructing a seed as discussed in Sections 8.6.1 and 8.6.2. In the specifications of this Recommendation, a Get_entropy_input function is used for this purpose. The entropy input and nonce shall be provided as discussed in Sections 8.6.5 and $\underline{8.6 .7}$ and in [SP 800-90C].

The Get_entropy_input function is specified in pseudocode in [SP 800-90C] for various RBG constructions; however, in general, the function has the following meaning:

Get_entropy_input: A function that is used to obtain entropy input. The function call is:

(status, entropy_input $)=$ Get_entropy_input (min_entropy, min_length, max_ length, prediction_resistance_request),

which requests a string of bits (entropy_input) with at least min_entropy bits of entropy. The length for the string shall be equal to or greater than min_length bits, and less than or equal to max_length bits. The prediction_resistance_request parameter indicates whether or not prediction resistance is to be provided during the request (i.e., whether fresh entropy is required $^{4}$ ). A status code is returned from the function.

Note that an implementation may choose to define this functionality differently by omitting some of the parameters; for example, for many of the DRBG mechanisms, min_length = min_entropy for the Get_entropy_input function, in which case, the second parameter could be omitted.

In the pseudocode in this section, two classes of error codes are returned: ERROR_FLAG and CATASTROPHIC_ERROR_FLAG. The handling of these classes of error codes is discussed in Section 11.4. The error codes may, in fact, provide information about the reason for the error; for example, when ERROR_FLAG is returned because of an incorrect input parameter, the ERROR_FLAG may indicate the problem.

Consuming applications should check the status returned from DRBG functions to determine whether or not the request was successful or if remediary action is required. For example, when

\footnotetext{
${ }^{4}$ Entropy input may be obtained from an entropy source or an NRBG, both of which provide fresh entropy. Entropy input could also be obtained from a DRBG that has access to an entropy source or NRBG.

The request for prediction resistance rules out the use of a DRBG that does not have access to either an entropy source or NRBG.
} 
the instantiate function returns an error, an instantiation will not have been created, and an invalid state_handle will be returned (see Section 9.1); however, the lack of a state_handle will be detected in a subsequent reseed or generate request. When the reseed function returns an error (see Section 9.2), the indicated instantiation will not have been reseeded (i.e., the internal state will not have been injected with fresh entropy). When the generate function returns an error, a null string is returned as the output string (see Section 9.3.1) and shall not be used as pseudorandom output.

Comments are often included in the pseudocode in this Recommendation. A comment placed on a line that includes pseudocode applies to that line; a comment placed on a line containing no pseudocode applies to one or more lines of pseudocode immediately below that comment.

\subsection{Instantiating a DRBG}

A DRBG shall be instantiated prior to the generation of pseudorandom bits. The instantiate function:

1. Checks the validity of the input parameters,

2. Determines the security strength for the DRBG instantiation,

3. Obtains entropy input with entropy sufficient to support the security strength,

4. Obtains the nonce (if required),

5. Determines the initial internal state using the instantiate algorithm, and

6. If an implementation supports multiple simultaneous instantiations of the same DRBG, a state_handle for the internal state is returned to the consuming application (see below).

Let working_state be the working state for the particular DRBG mechanism (e.g.,

HMAC_DRBG), and let min_length, max_length, and highest_supported_security_strength be defined for each DRBG mechanism (see Section 10). Let Instantiate_algorithm be a call to the appropriate instantiate algorithm for the DRBG mechanism (see Section 10).

The following or an equivalent process shall be used to instantiate a DRBG.

Instantiate_function (requested_instantiation_security_strength, prediction_resistance_flag, personalization_string):

1. requested_instantiation_security_strength: A requested security strength for the instantiation. Implementations that support only one security strength do not require this parameter; however, any consuming application using that implementation must be aware of the security strength that is supported.

2. prediction_resistance_flag: Indicates whether or not prediction resistance may be required by the consuming application during one or more requests for pseudorandom bits. Implementations that always provide or do not support prediction resistance may not need to support this parameter if the intent is implicitly known. However, the user of a consuming application must determine whether or not prediction resistance may be required by the consuming application before electing to use such an implementation. If the prediction_resistance_flag is not needed (i.e., it is known that prediction resistance is always performed or is not supported), then the prediction_resistance_flag input 
parameter and instantiate process step 2 are omitted, and the prediction_resistance_flag is omitted from the internal state in step 11 of the instantiate process. In addition, step 6 can be modified to not perform a check for the prediction_resistance_flag when the flag is not used in an implementation; in this case, the Get_entropy_input call need not include the prediction_resistance_request parameter.

3. personalization_string: An optional input that provides personalization information (see Sections 8.6.1 and 8.7.1). The maximum length of the personalization string (max_personalization_string_length) is implementation dependent, but shall be less than or equal to the maximum length specified for the given DRBG mechanism (see Section 10). If the input of a personalization string is not supported, then the personalization_string input parameter and step 3 of the instantiate process are omitted, and instantiate process step 9 is modified to omit the personalization string.

\section{Required information not provided by the consuming application during instantiation} (This information shall not be provided by the consuming application as an input parameter during the instantiate request):

1. entropy_input: Input bits containing entropy. The maximum length of the entropy_input is implementation dependent, but shall be less than or equal to the specified maximum length for the selected DRBG mechanism (see Section 10).

2. nonce: A nonce as specified in Section 8.6.7. Note that if a random value is used in the nonce, the entropy_input and random portion of the nonce could be acquired using a single Get_entropy_input call (see step 6 of the instantiate process); in this case, the first parameter of the Get_entropy_input call is adjusted to include the entropy for the nonce (i.e., the security_strength is increased by at least $1 / 2$ security_strength, and minlength is increased to accommodate the length of the nonce), instantiate process step 8 is omitted, and the nonce is omitted from the parameter list in instantiate process step 9.

Note that in some cases, a nonce will not be used by a DRBG mechanism; in this case, step 8 is omitted, and the nonce is omitted from the parameter list in instantiate process step 9.

\section{Output to a consuming application after instantiation:}

1. status: The status returned from the instantiate function. If any status other than SUCCESS is returned, either no state_handle or an invalid state_handle shall be returned to the consuming application. A consuming application should check the status to determine that the DRBG has been correctly instantiated.

2. state_handle: Used to identify the internal state for this instantiation in subsequent calls to the generate, reseed, uninstantiate and health test functions.

If a state handle is not required for an implementation because the implementation does not support multiple simultaneous instantiations, a state_handle need not be returned. In this case, instantiate process step 10 is omitted, process step 11 is revised to save the only internal state, and process step 12 is altered to omit the state_handle.

Information retained within the DRBG mechanism boundary after instantiation: 
The internal state for the DRBG, including the working_state and administrative information (see Sections 8.3 and 10 for definitions of the working_state and administrative information).

\section{Instantiate Process:}

Comment: Check the validity of the input parameters.

1. If requested_instantiation_security_strength> highest_supported_security_strength, then return (ERROR_FLAG, Invalid).

2. If prediction_resistance_flag is set, and prediction resistance is not supported, then return (ERROR_FLAG, Invalid).

3. If the length of the personalization_string $>$ max_personalization_string_length, return (ERROR_FLAG, Invalid).

4. Set security_strength to the lowest security strength greater than or equal to requested_instantiation_security_strength from the set $\{112,128,192,256\}$.

5. Null step.

Comment: This null step replaces a step from the original version of SP 800-90 without changing the step numbers.

Comment: Obtain the entropy input.

6. (status, entropy_input) $=$ Get_entropy_input (security_strength, min_length, max_length, prediction_resistance_request).

Comment: status indications other than SUCCESS could be ERROR_FLAG or CATASTROPHIC_ERROR_FLAG, in which case, the status is returned to the consuming application to handle. The Get_entropy_input call could return a status of ERROR_FLAG to indicate that entropy is currently unavailable, and could return CATASTROPHIC_ERROR_FLAG to indicate that an entropy source failed.

7. If (status $\neq$ SUCCESS), return (status, Invalid).

8. Obtain a nonce.

Comment: This step shall include any appropriate checks on the acceptability of the nonce. See Section 8.6.7.

Comment: Call the appropriate instantiate algorithm in Section 10 to obtain values for the initial working_state.

9. initial_working_state $=$ Instantiate_algorithm (entropy_input, nonce, personalization_string, security_strength).

10. Get a state_handle for a currently empty internal state. If an empty internal state cannot be found, return (ERROR_FLAG, Invalid). 
11. Set the internal state for the new instantiation (e.g., as indicated by state_handle) to the initial values for the internal state (i.e., set the working_state to the values returned as initial_working_state in step 9) and any other values required for the working_state (see Section 10), and set the administrative information to the appropriate values (e.g., the values of security_strength and the prediction_resistance_flag).

12. Return (SUCCESS, state_handle).

\subsection{Reseeding a DRBG Instantiation}

The reseeding of an instantiation is not required, but is recommended whenever a consuming application and implementation are able to perform this process. Reseeding will insert additional entropy into the generation of pseudorandom bits. Reseeding may be:

- Explicitly requested by a consuming application,

- Performed when prediction resistance is requested by a consuming application,

- Triggered by the generate function when a predetermined number of pseudorandom outputs have been produced or a predetermined number of generate requests have been made (i.e., at the end of the seedlife), or

- Triggered by external events (e.g., whenever entropy is available).

The reseed function:

1. Checks the validity of the input parameters,

2. Obtains entropy input from a randomness source that supports the security strength of the DRBG, and

3. Using the reseed algorithm, combines the current working state with the new entropy input and any additional input to determine the new working state.

Let working_state be the working state for the particular DRBG instantiation (e.g., HMAC_DRBG), let min_length and max_length be defined for each DRBG mechanism, and let Reseed_algorithm be a call to the appropriate reseed algorithm for the DRBG mechanism (see Section 10).

The following or an equivalent process shall be used to reseed the DRBG instantiation.

Reseed_function (state_handle,prediction_resistance_request, additional_input):

1) state_handle: A pointer or index that indicates the internal state to be reseeded. If a state handle is not used by an implementation because the implementation does not support multiple simultaneous instantiations, a state_handle is not provided as input. Since there is only a single internal state in this case, reseed process step 1 obtains the contents of the internal state, and reseed process step 6 replaces the working_state of this internal state. 
2) prediction_resistance_request: Indicates whether or not prediction resistance is to be provided during the request (i.e., whether or not fresh entropy bits are required) 5 . Without the explicit prediction resistance request, the entropy input could be provided from either a DRBG with no access to an entropy source (i.e., fresh entropy would not be provided), or the entropy input could be provided by an entropy source or by an RBG with access to an entropy source (i.e., fresh entropy would be provided in these cases).

DRBGs that are implemented to always support prediction resistance or to never support prediction resistance do not require this parameter. However, when prediction resistance is not supported, the user of a consuming application must determine whether or not prediction resistance may be required by the application before electing to use such a DRBG implementation.

If prediction resistance is not supported, then the prediction_resistance_request input parameter and step 2 of the reseed process is omitted, and reseed process step 4 is modified to omit the prediction_resistance_request parameter.

If prediction resistance is always performed, then the prediction_resistance_request input parameter and reseed process step 2 may be omitted, and reseed process step 4 is replaced by:

$($ status, entropy_input $)=$ Get_entropy_input (security_strength, min_length, max_length)

3) additional_input: An optional input. The maximum length of the additional_input (max_additional_input_length) is implementation dependent, but shall be less than or equal to the maximum value specified for the given DRBG mechanism (see Section 10). If the input by a consuming application of additional_input is not supported, then the input parameter and step 2 of the reseed process are omitted, and step 5 of the reseed process is modified to remove the additional_input from the parameter list.

\section{Required information not provided by the consuming application during reseeding (This} information shall not be provided by the consuming application as an input parameter during the reseed request):

1. entropy_input: Input bits containing entropy. This input shall not be provided by the DRBG instantiation being reseeded. The maximum length of the entropy_input is implementation dependent, but shall be less than or equal to the specified maximum length for the selected DRBG mechanism (see Section 10).

2. Internal state values required by the DRBG for the working_state and administrative information, as appropriate.

\section{Output to a consuming application after reseeding:}

1. status: The status returned from the function.

${ }^{5}$ A DRBG may be reseeded by an entropy source or an NRBG, both of which provide fresh entropy. A DRBG could also be reseeded by a DRBG that has access to an entropy source or NRBG. The request for prediction resistance during reseeding rules out the use of a DRBG that does not have access to either an entropy source or NRBG. See [SP 800-90C] for further discussion. 


\section{Information retained within the DRBG mechanism boundary after reseeding:}

Replaced internal state values (i.e., the working_state).

\section{Reseed Process:}

Comment: Get the current internal state and check the input parameters.

1. Using state_handle, obtain the current internal state. If state_handle indicates an invalid or unused internal state, return (ERROR_FLAG).

2. If prediction_resistance_request is set, and prediction_resistance_flag is not set, then return (ERROR_FLAG).

3. If the length of the additional_input $>$ max_additional_input_length, return (ERROR_FLAG).

Comment: Obtain the entropy input.

4. (status, entropy_input) $=$ Get_entropy_input (security_strength, min_length, max_length, prediction_resistance_request).

Comment: status indications other than SUCCESS could be ERROR_FLAG or CATASTROPHIC_ERROR_FLAG, in which case, the status is returned to the consuming application to handle. The Get_entropy_input call could return a status of ERROR_FLAG to indicate that entropy is currently unavailable, and could return CATASTROPHIC_ERROR_FLAG to indicate that an entropy source failed.

5. If (status $\neq$ SUCCESS), return (status).

Comment: Get the new working_state using the appropriate reseed algorithm in Section 10.

6. new_working_state $=$ Reseed_algorithm (working_state, entropy_input, additional_input).

7 Replace the working_state in the internal state for the DRBG instantiation (e.g., as indicated by state_handle) with the values of new_working_state obtained in step 6.

8. Return (SUCCESS).

\subsection{Generating Pseudorandom Bits Using a DRBG}

This function is used to generate pseudorandom bits after instantiation or reseeding. The generate function:

1. Checks the validity of the input parameters.

2. Calls the reseed function to obtain sufficient entropy if the instantiation needs additional entropy because the end of the seedlife has been reached or prediction resistance is 
required; see Sections 9.3.2 and 9.3.3 for more information on reseeding at the end of the seedlife and on handling prediction resistance requests.

3. Generates the requested pseudorandom bits using the generate algorithm.

4. Updates the working state.

5. Returns the requested pseudorandom bits to the consuming application.

\subsubsection{The Generate Function}

Let outlen be the length of the output block of the cryptographic primitive (see Section 10). Let Generate_algorithm be a call to the appropriate generate algorithm for the DRBG mechanism (see Section 10), and let Reseed_function be a call to the reseed function in Section 9.2.

The following or an equivalent process shall be used to generate pseudorandom bits.

Generate_function (state_handle, requested_number_of_bits, requested_security_strength, prediction_resistance_request, additional_input):

1. state_handle: A pointer or index that indicates the internal state to be used. If a state handle is not used by an implementation because the implementation does not support multiple simultaneous instantiations, a state_handle is not provided as input. The state_handle is then omitted from the input parameter list in process step 7.1, generate process steps 1 and 7.3 are used to obtain the contents of the internal state, and process step 10 replaces the working_state of this internal state.

2. requested_number_of_bits: The number of pseudorandom bits to be returned from the generate function. The max_number_of_bits_per_request is implementation dependent, but shall be less than or equal to the value provided in Section 10 for a specific DRBG mechanism.

3. requested_security_strength: The security strength to be associated with the requested pseudorandom bits. DRBG implementations that support only one security strength do not require this parameter; however, any consuming application using that DRBG implementation must be aware of the supported security strength.

4. prediction_resistance_request: Indicates whether or not prediction resistance is to be provided during the request. DRBGs that are implemented to always provide prediction resistance or that do not support prediction resistance do not require this parameter. However, when prediction resistance is not supported, the user of a consuming application must determine whether or not prediction resistance may be required by the application before electing to use such a DRBG implementation.

If prediction resistance is not supported, then the prediction_resistance_request input parameter and steps 5 and 9.2 of the generate process are omitted, and generate process steps 7 and 7.1 are modified to omit the check for the prediction_resistance_request term.

If prediction resistance is always performed, then the prediction_resistance_request input parameter and generate process steps 5 and 9.2 may be omitted, and generate process steps 7 and 8 may be replaced by:

status $=$ Reseed_function (state_handle, additional_input). 
Comment: status indications other than SUCCESS could be ERROR_FLAG or CATASTROPHIC_ERROR_FLAG, in which case, the status is returned to the consuming application to handle. The Get_entropy_input call could return a status of ERROR FLAG to indicate that entropy is currently unavailable, and could return CATASTROPHIC_ERROR_FLAG to indicate that an entropy source failed.

If (status $\neq$ SUCCESS), return (status, Null).

Using state_handle, obtain the new internal state.

$($ status, pseudorandom_bits, new_working_state $)=$ Generate_algorithm (working_state, requested_number_of_bits).

Note that if the input of additional_input is not supported, then the additional_input parameter in the Reseed_function call above may be omitted.

5. additional_input: An optional input. The maximum length of the additional_input (max_additional_input_length) is implementation dependent, but shall be less than or equal to the specified maximum length for the selected DRBG mechanism (see Section 10). If the input of additional_input is not supported, then the input parameter, generate process steps 4 and 7.4, and the additional_input input parameter in generate process steps 7.1 and 8 are omitted.

\section{Required information not provided by the consuming application during generation:}

1. Internal state values required for the working_state and administrative information, as appropriate.

\section{Output to a consuming application after generation:}

1. status: The status returned from the generate function. If any status other than SUCCESS is returned, a Null string shall be returned as the pseudorandom bits.

2. pseudorandom_bits: The pseudorandom bits that were requested or a Null string.

Information retained within the DRBG mechanism boundary after generation:

Replaced internal state values (i.e., the new working_state).

\section{Generate Process:}

Comment: Get the internal state and check the input parameters.

1. Using state_handle, obtain the current internal state for the instantiation. If state_handle indicates an invalid or unused internal state, then return (ERROR_FLAG, Null).

2. If requested_number_of_bits $>$ max_number_of_bits_per_request, then return (ERROR_FL̄AG, Null).

3. If requested_security_strength $>$ the security_strength indicated in the internal state, then return (ERROR_FLAG, Null). 
4. If the length of the additional_input $>$ max_additional_input_length, then return (ERROR_FLAG, Null).

5. If prediction_resistance_request is set, and prediction_resistance_flag is not set, then return (ERRŌR_FLAG, Null).

6. Clear the reseed_required_flag. Comment: See Section 9.3.2 for a discussion.

Comment: Reseed if necessary (see Section 9.2).

7. If reseed_required_flag is set, or if prediction_resistance_request is set, then

7.1 $s t a t u s=$ Reseed_function (state_handle, prediction_resistance_request, additional_input).

Comment: status indications other than SUCCESS could be ERROR_FLAG or CATASTROPHIC_ERROR_FLAG, in which case, the status is returned to the consuming application to handle. The Get_entropy_input call could return a status of ERROR_FLAG to indicate that entropy is currently unavailable, and could return CATASTROPHIC_ERROR_FLAG to indicate that an entropy source failed.

7.2 If (status $\neq$ SUCCESS), then return (status, Null).

7.3 Using state_handle, obtain the new internal state.

7.4 additional_input $=$ the Null string.

7.5 Clear the reseed_required_flag.

Comment: Request the generation of pseudorandom_bits using the appropriate generate algorithm in Section 10.

8. (status, pseudorandom_bits, new_working_state $)=$ Generate_algorithm (working_state, requested_number_of_bits, additional_input).

9. If status indicates that a reseed is required before the requested bits can be generated, then

9.1 Set the reseed_required_flag.

9.2 If the prediction_resistance_flag is set, then set the prediction_resistance request indication.

9.3 Go to step 7.

10. Replace the old working_state in the internal state of the DRBG instantiation (e.g., as indicated by state_handle) with the values of new_working_state.

11. Return (SUCCESS, pseudorandom_bits).

Implementation notes: 
If a reseed capability is not supported, or a reseed is not desired, then generate process steps 6 and 7 are removed; generate process step 9 is replaced by:

9. If status indicates that a reseed is required before the requested bits can be generated, then

9.1 status $=$ Uninstantiate_function (state_handle).

9.2 Return an indication that the DRBG instantiation can no longer be used.

\subsubsection{Reseeding at the End of the Seedlife}

When pseudorandom bits are requested by a consuming application, the generate function checks whether or not a reseed is required by comparing the counter within the internal state (see Section 8.3) against a predetermined reseed interval for the DRBG implementation. This is specified in the generate process (see Section 9.3.1) as follows:

a. Step 6 clears the reseed_required_flag.

b. Step 7 checks the value of the reseed_required_flag. At this time, the reseed_required_flag is clear, so step 7 is skipped unless prediction resistance was requested by the consuming application. For the purposes of this explanation, assume that prediction resistance was not requested.

c. Step 8 calls the Generate_algorithm, which checks whether a reseed is required. If it is required, an appropriate status is returned.

d. Step 9 checks the status returned by the Generate_algorithm. If the status does not indicate that a reseed is required, the generate process continues with step 10 .

e. However, if the status indicates that a reseed is required (see step 9), then the reseed_required_flag is set, the prediction_resistance_request indicator is set if the instantiation is capable of performing prediction resistance, and processing continues by going back to step 7. This is intended to obtain fresh entropy for reseeding at the end of the reseed interval whenever access to fresh entropy is available (see the concept of Live Entropy Sources in [SP 800-90C]).

f. The substeps in step 7 are executed. The reseed function is called; any additional_input provided by the consuming application in the generate request is used during reseeding. The new values of the internal state are acquired, any additional input provided by the consuming application in the generate request is replaced by a Null string, and the reseed_required_flag is cleared.

g. The generate algorithm is called (again) in step 8, the check of the returned status is made in step 9, and (presumably) step 10 is then executed.

\subsubsection{Handling Prediction Resistance Requests}

When pseudorandom bits are requested by a consuming application with prediction resistance, the generate function specified in Section 9.3.1 checks that the instantiation allows prediction resistance requests (see step 5 of the generate process); clears the reseed_required_flag (even though the flag won't be used in this case); executes the substeps of generate process step 7, resulting in a reseed, a new internal state for the instantiation, and setting the additional input to a 
Null value; obtains pseudorandom bits (see generate process step 8); passes through generate process step 9, since another reseed will not be required; and continues with generate process step 10.

\subsection{Removing a DRBG Instantiation}

The internal state for an instantiation may need to be "released" by erasing (i.e., zeroizing) the contents of the internal state. The uninstantiate function:

1. Checks the input parameter for validity, and

2. Empties the internal state.

The following or an equivalent process shall be used to remove (i.e., uninstantiate) a DRBG instantiation:

\section{Uninstantiate_function (state_handle):}

1. state_handle: A pointer or index that indicates the internal state to be "released". If a state handle is not used by an implementation because the implementation does not support multiple simultaneous instantiations, a state_handle is not provided as input. In this case, uninstantiate process step 1 is omitted, and process step 2 erases the internal state.

\section{Output to a consuming application after uninstantiation:}

1. status: The status returned from the function.

Information retained within the DRBG mechanism boundary after uninstantiation:

1. An empty internal state.

\section{Uninstantiate Process:}

1. If state_handle indicates an invalid state, then return (ERROR_FLAG).

2. Erase the contents of the internal state indicated by state_handle.

3. Return (SUCCESS). 


\section{DRBG Algorithm Specifications}

Several DRBG mechanisms are specified in this Recommendation. The selection of a DRBG mechanism depends on several factors, including the security strength to be supported and what cryptographic primitives are available. An analysis of the consuming application's requirements for random numbers should be conducted in order to select an appropriate DRBG mechanism. Conversion specifications required for the DRBG mechanism implementations (e.g., between integers and bitstrings) are provided in Appendix A. Pseudocode examples for each DRBG mechanism are provided in Appendix B. A detailed discussion on DRBG mechanism selection is provided in Appendix C.

Examples for determining correct implementation of each DRBG are available at http://csrc.nist.gov/groups/ST/toolkit/examples.html.

\subsection{DRBG Mechanisms Based on Hash Functions}

A DRBG mechanism may be based on a hash function that is one-way. The hash-based DRBG mechanisms specified in this Recommendation have been designed to use any approved hash function and may be used by consuming applications requiring various security strengths, providing that the appropriate hash function is used and sufficient entropy is obtained for the seed.

The following are provided as DRBG mechanisms based on hash functions:

1. The Hash_DRBG specified in Section 10.1.1.

2. The HMAC_DRBG specified in Section 10.1.2.

The maximum security strength that can be supported by each DRBG based on a hash function is the security strength of the hash function for pre-image resistance; these security strengths are provided in [SP 800-107]. [SP 800-57] identifies hash functions that can be used to support a required security strength.

This Recommendation supports only four security strengths: 112, 128, 192, and 256 bits. Table 2 specifies the values that shall be used for the function envelopes ${ }^{6}$ and DRBG algorithm for each approved hash function.

\footnotetext{
${ }^{6}$ Discussed in Section 9.
} 
Table 2: Definitions for Hash-Based DRBG Mechanisms

\begin{tabular}{|c|c|c|c|c|c|}
\hline & SHA-1 & $\begin{array}{l}\text { SHA-224 } \\
\text { and SHA- } \\
512 / 224\end{array}$ & $\begin{array}{l}\text { SHA-256 } \\
\text { and } \\
\text { SHA- } \\
\mathbf{5 1 2 / 2 5 6}\end{array}$ & SHA-384 & SHA-512 \\
\hline Supported security strengths & \multicolumn{5}{|c|}{ See [SP 800-57] } \\
\hline highest_supported_security_strength & \multicolumn{5}{|c|}{ See [SP 800-57] } \\
\hline Output Block Length (outlen) & 160 & 224 & 256 & 384 & 512 \\
\hline $\begin{array}{l}\text { Required minimum entropy for } \\
\text { instantiate and reseed }\end{array}$ & \multicolumn{5}{|c|}{ security_strength } \\
\hline $\begin{array}{l}\text { Minimum entropy input length } \\
\text { (min_length) }\end{array}$ & \multicolumn{5}{|c|}{ security_strength } \\
\hline $\begin{array}{l}\text { Maximum entropy input length } \\
\text { (max_length) }\end{array}$ & \multicolumn{5}{|c|}{$2^{35}$ bits } \\
\hline $\begin{array}{l}\text { Seed length (seedlen) for } \\
\text { Hash_DRBG }\end{array}$ & 440 & 440 & 440 & 888 & 888 \\
\hline $\begin{array}{l}\text { Maximum personalization string } \\
\text { length } \\
\text { (max_personalization_string_length) }\end{array}$ & \multicolumn{5}{|c|}{$2^{35}$ bits } \\
\hline $\begin{array}{l}\text { Maximum additional_input length } \\
\text { (max_additional_input_length) }\end{array}$ & \multicolumn{5}{|c|}{$2^{35}$ bits } \\
\hline max_number_of_bits_per_request & \multicolumn{5}{|c|}{$2^{19}$ bits } \\
\hline $\begin{array}{l}\text { Maximum number of requests } \\
\text { between reseeds (reseed_interval) }\end{array}$ & \multicolumn{5}{|c|}{$2^{48}$} \\
\hline
\end{tabular}

Note that since SHA-224 is based on SHA-256, there is no efficiency benefit when using SHA224, rather than SHA-256. Also note that since SHA-384, SHA-512/224 and SHA-512/256 are based on SHA-512, there is no efficiency benefit for using these three SHA mechanisms, rather than using SHA-512. However, efficiency is just one factor to consider when selecting the appropriate hash function to use as part of a DRBG mechanism.

\subsubsection{Hash_DRBG}

Figure 8 presents the normal operation of the Hash_DRBG generate algorithm. The Hash_DRBG requires the use of a hash function during the instantiate, reseed and generate functions; the same hash function shall be used throughout a Hash_DRBG instantiation. Hash_DRBG uses the derivation function specified in Section 10.3.1 during instantiation and reseeding. The security strength of the hash function to be used shall meet or exceed the desired security strength required by the consuming application for DRBG output (see [SP 800-57]). 


\subsubsection{Hash_DRBG Internal State}

The internal_state for Hash_DRBG consists of:

1. The working_state:

a. A value $(V)$ of seedlen bits that is updated during each call to the DRBG.

b. A constant $(C)$ of seedlen bits that depends on the seed.

c. A counter (reseed_counter) that indicates the number of requests for pseudorandom bits since new entropy_input was obtained during instantiation or reseeding.

2. Administrative information:

a. The security_strength of the DRBG instantiation.

b. A prediction_resistance_flag that indicates whether or not a prediction resistance capability is available for the DRBG instantiation.

The values of $V$ and $C$ are the critical values of the internal state upon which the security of this DRBG mechanism depends (i.e., $V$ and $C$ are the "secret values" of the internal state).

\subsubsection{Instantiation of Hash DRBG}

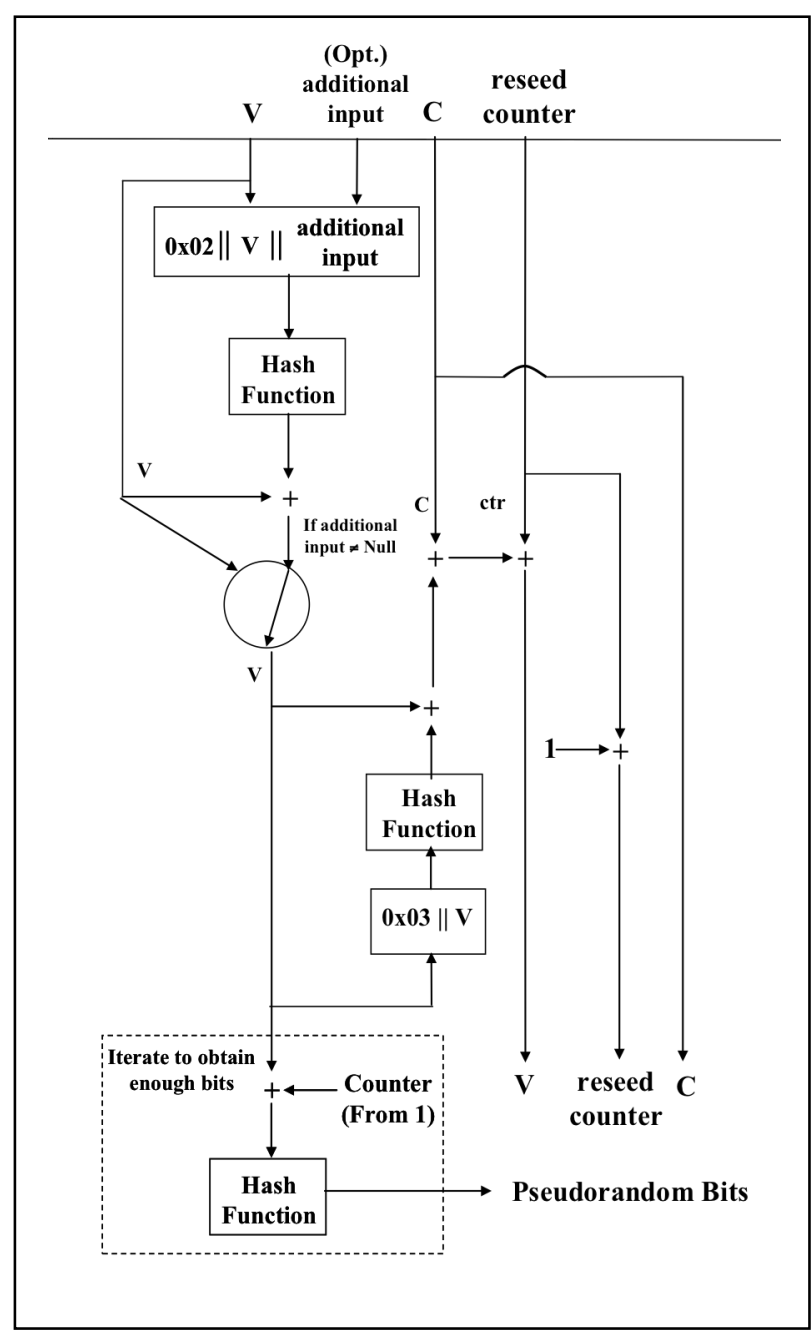

Figure 8: Hash_DRBG

Notes for the instantiate function specified in Section 9.1:

The instantiation of Hash_DRBG requires a call to the Instantiate_function specified in Section 9.1. Process step 9 of that function calls the instantiate algorithm in this section.

The values of highest_supported_security_strength and min_length are provided in Table 2 of Section 10.1. The contents of the internal state are provided in Section 10.1.1.1.

The instantiate algorithm:

Let Hash_df be the hash derivation function specified in Section 10.3.1 using the selected hash function. The output block length (outlen), seed length (seedlen) and appropriate security_strengths for the implemented hash function are provided in Table 2 of Section 10.1 . 
The following process or its equivalent shall be used as the instantiate algorithm for this DRBG mechanism (see step 9 of the instantiate process in Section 9.1).

Hash_DRBG_Instantiate_algorithm (entropy_input, nonce, personalization_string, security_strength):

1. entropy_input: The string of bits obtained from the randomness source.

2. nonce: A string of bits as specified in Section 8.6.7.

3. personalization_string: The personalization string received from the consuming application. Note that the length of the personalization_string may be zero.

4. security_strength: The security strength for the instantiation. This parameter is optional for Hash_DRBG, since it is not used.

\section{Output:}

1. initial_working_state: The initial values for $V, C$, and reseed_counter (see Section 10.1.1.1).

\section{Hash_DRBG Instantiate Process:}

1. seed_material $=$ entropy_input $\|$ nonce $\|$ personalization_string.

2. seed=Hash_df (seed_material, seedlen).

3. $V=$ seed.

4. $C=$ Hash_df $((0 x 00 \| V)$, seedlen $)$. Comment: Precede $V$ with a byte of zeros.

5. reseed_counter $=1$.

6. Return $(V, C$, reseed_counter $)$.

\subsubsection{Reseeding a Hash_DRBG Instantiation}

Notes for the reseed function specified in Section 9.2:

The reseeding of a Hash_DRBG instantiation requires a call to the Reseed_function specified in Section 9.2. Process step 6 of that function calls the reseed algorithm specified in this section. The values for min_length are provided in Table 2 of Section 10.1.

The reseed algorithm:

Let Hash_df be the hash derivation function specified in Section 10.3.1 using the selected hash function. The value for seedlen is provided in Table 2 of Section 10.1.

The following process or its equivalent shall be used as the reseed algorithm for this DRBG mechanism (see step 6 of the reseed process in Section 9.2):

Hash_DRBG_Reseed_algorithm (working_state, entropy_input, additional_input):

1. working_state: The current values for $V, C$, and reseed_counter (see Section 10.1.1.1).

2. entropy_input: The string of bits obtained from the randomness source. 
3. additional_input: The additional input string received from the consuming application. Note that the length of the additional_input string may be zero.

\section{Output:}

1. new_working_state: The new values for $V, C$, and reseed counter.

\section{Hash_DRBG Reseed Process:}

1. seed_material $=0 \times 01\|V\|$ entropy_input $\|$ additional_input.

2. seed=Hash_df (seed_material, seedlen).

3. $V=$ seed.

4. $\quad C=$ Hash_df $((0 \mathrm{x} 00 \| V)$, seedlen $)$. Comment: Preceed with a byte of all zeros.

5. reseed_counter $=1$.

6. Return $(V, C$, and reseed_counter).

\subsubsection{Generating Pseudorandom Bits Using Hash_DRBG}

Notes for the generate function specified in Section 9.3:

The generation of pseudorandom bits using a Hash_DRBG instantiation requires a call to the generate function specified in Section 9.3. Process step 8 of that function calls the generate algorithm specified in this section. The values for max_number_of_bits_per_request and outlen are provided in Table 2 of Section 10.1.

The generate algorithm:

Let Hash be the selected hash function. The seed length (seedlen) and the maximum interval between reseeding (reseed_interval) are provided in Table 2 of Section 10.1. Note that for this DRBG mechanism, the reseed counter is used to update the value of $V$, as well as to count the number of generation requests.

The following process or its equivalent shall be used as the generate algorithm for this DRBG mechanism (see step 8 of the generate process in Section 9.3):

Hash_DRBG_Generate_algorithm (working_state, requested_number_of_bits, additional_input):

1. working_state: The current values for $V, C$, and reseed_counter (see Section 10.1.1.1).

2. requested_number_of_bits: The number of pseudorandom bits to be returned to the generate function.

3. additional_input: The additional input string received from the consuming application. Note that the length of the additional_input string may be zero.

\section{Output:}


1. status: The status returned from the function. The status will indicate SUCCESS, or indicate that a reseed is required before the requested pseudorandom bits can be generated.

2. returned_bits: The pseudorandom bits to be returned to the generate function.

3. new_working_state: The new values for $V, C$, and reseed_counter.

\section{Hash_DRBG_Generate Process:}

1. If reseed_counter > reseed_interval, then return an indication that a reseed is required.

2. If (additional_input $\neq N u l l)$, then do

$2.1 w=$ Hash $(0 x 02\|V\|$ additional_input).

$2.2 V=(V+w) \bmod 2^{\text {seedlen }}$.

3. (returned_bits) $=$ Hashgen $($ requested_number_of_bits, $V)$.

4. $H=\operatorname{Hash}(0 \mathrm{x} 03 \| V)$.

5. $V=(V+H+C+$ reseed_counter $) \bmod 2^{\text {seedlen }}$.

6. reseed_counter $=$ reseed_counter +1 .

7. Return (SUCCESS, returned_bits, $V, C$, reseed_counter).

Hashgen (requested_number_of_bits, V):

\section{Input:}

1. requested_no_of_bits: The number of bits to be returned.

2. $V$ : The current value of $V$.

\section{Output:}

1. returned_bits: The generated bits to be returned to the generate function.

\section{Hashgen Process:}

1. $m=\left\lceil\frac{\text { requested_no_of_bits }}{\text { outlen }}\right\rceil$.

2. $\operatorname{data}=V$.

3. $W=$ the Null string.

4. For $i=1$ to $m$

$4.1 w=$ Hash $($ data $)$.

$4.2 W=W \| w$.

4.3 data $=($ data +1$) \bmod 2^{\text {seedlen }}$.

5. returned_bits $=$ leftmost $(W$,requested_no_of_bits $)$.

6. Return (returned_bits). 


\subsubsection{HMAC_DRBG}

HMAC_DRBG uses multiple occurrences of an approved keyed hash function, which is based on an approved hash function. This DRBG mechanism uses the

HMAC_DRBG_Update function specified in Section 10.1.2.2 and the HMAC function within the HMAC_DRBG_Update function as the derivation function during instantiation and reseeding. The same hash function shall be used throughout an HMAC_DRBG instantiation. The hash function used shall meet or exceed the security strength required by the consuming application for DRBG output (see [SP 800-57]).

Figure 9 depicts the HMAC_DRBG in three stages. HMAC_DRBG is specified using an internal function

(HMAC_DRBG_Update). This function is called during the HMAC_DRBG instantiate, generate and reseed algorithms to adjust the internal state when new entropy or additional input is provided, as well as to update the internal state after pseudorandom bits are generated. The operations in the top portion of the figure are only performed if the additional input is not null. Figure 10 depicts the HMAC_DRBG_Update function.

\subsubsection{HMAC_DRBG Internal State}

The internal state for HMAC_DRBG consists of:

1. The working_state:

a. The value $V$ of outlen bits, which is updated each time another outlen bits of output are produced (where outlen is specified in Table 2 of Section 10.1).

b. The outlen-bit Key, which is updated at least once each time that the DRBG mechanism generates pseudorandom bits.

c. A counter (reseed_counter) that

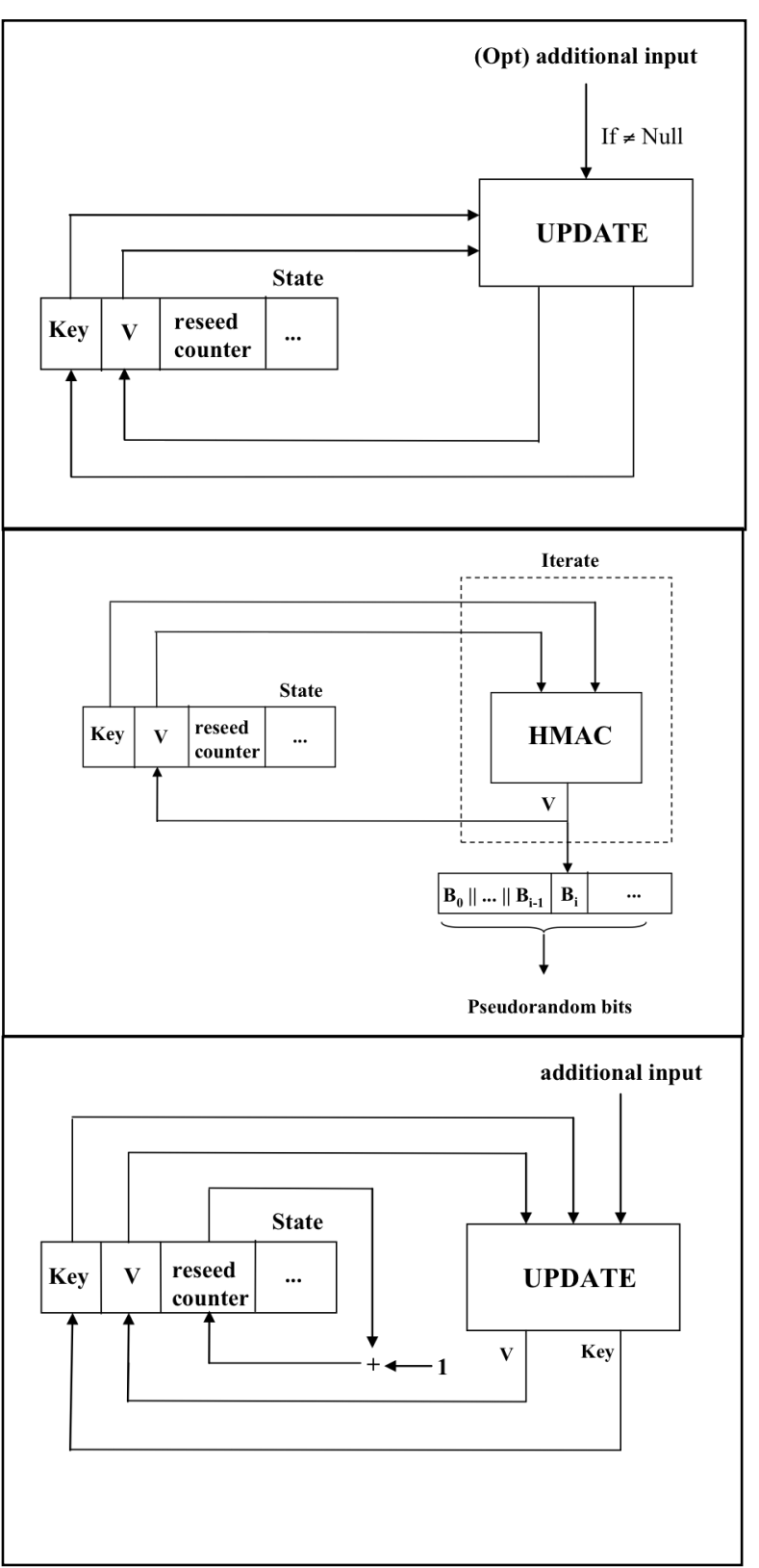

Figure 9: HMAC_DRBG Generate Function indicates the number of requests for pseudorandom bits since instantiation or reseeding. 
2. Administrative information:

a. The security_strength of the DRBG instantiation.

b. A prediction_resistance_flag that indicates whether or not a prediction resistance capability is required for the DRBG instantiation.

The values of $V$ and Key are the critical values of the internal state upon which the security of this DRBG mechanism depends (i.e., $V$ and Key are the "secret values" of the internal state).

\subsubsection{The HMAC_DRBG Update Function (Update)}

The HMAC_DRBG_Update function updates the internal state of

HMAC_DRBG using the provided_data. Note that for this DRBG mechanism, the

HMAC_DRBG_Update function also serves as a derivation function for the

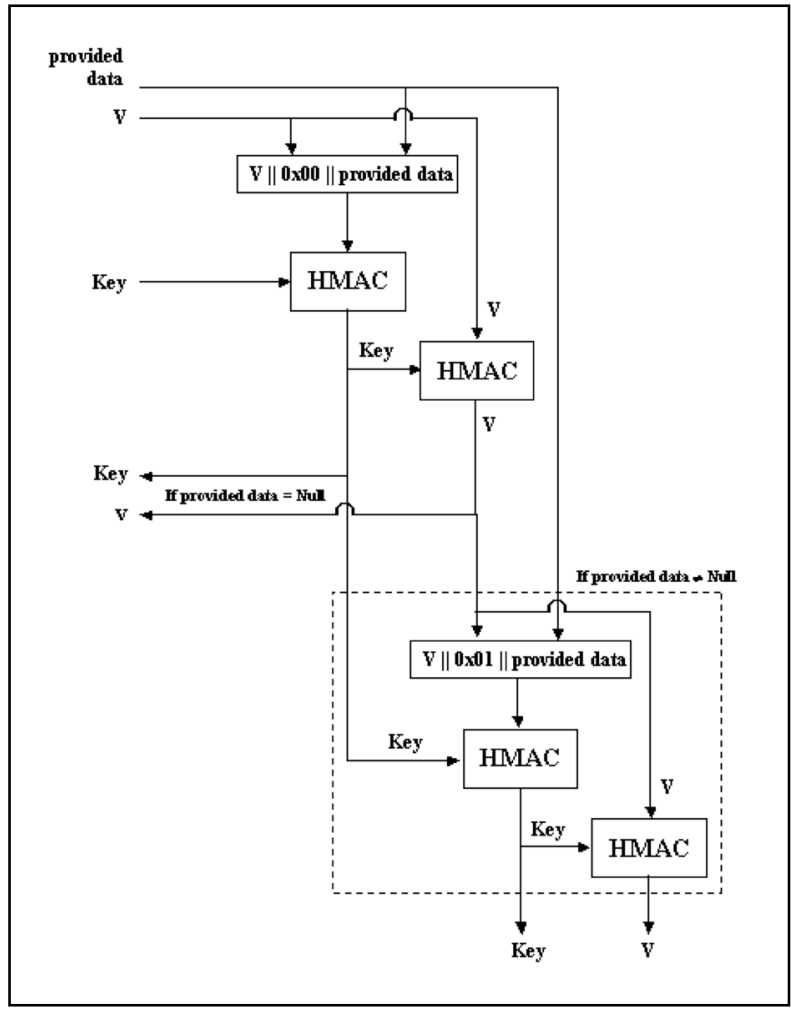

Figure 10: HMAC_DRBG_Update Function instantiate and reseed functions.

Let HMAC be the keyed hash function specified in [FIPS 198] using the hash function selected for the DRBG mechanism from Table 2 in Section 10.1.

The following or an equivalent process shall be used as the HMAC_DRBG_Update function.

HMAC_DRBG_Update (provided_data, $K, V$ ):

1. provided_data: The data to be used.

2. $K$ : The current value of $K e y$.

3. $V$ : The current value of $V$.

\section{Output:}

1. $K$ : The new value for Key.

2. $V$ : The new value for $V$.

HMAC_DRBG Update Process:

1. $K=$ HMAC $(K, V\|0 \times 00\|$ provided_data $)$.

2. $V=$ HMAC $(K, V)$. 
3. If (provided_data $=N u l l)$, then return $K$ and $V$.

4. $K=$ HMAC $(K, V\|0 \mathrm{x} 01\|$ provided_data $)$.

5. $V=$ HMAC $(K, V)$.

6. Return $(K, V)$.

\subsubsection{Instantiation of HMAC_DRBG}

Notes for the instantiate function specified in Section 9.1:

The instantiation of HMAC_DRBG requires a call to the Instantiate_function specified in Section 9.1. Process step 9 of that function calls the instantiate algorithm specified in this section. The values of highest_supported_security_strength and min length are provided in Table 2 of Section 10.1. The contents of the internal state are provided in Section 10.1.2.1.

The instantiate algorithm:

Let HMAC_DRBG_Update be the function specified in Section 10.1.2.2. The output block length (outlen) is provided in Table 2 of Section 10.1.

The following process or its equivalent shall be used as the instantiate algorithm for this DRBG mechanism (see step 9 of the instantiate process in Section 9.1):

HMAC_DRBG_Instantiate_algorithm (entropy_input, nonce, personalization_string, security_strength):

1. entropy_input: The string of bits obtained from the randomness source.

2. nonce: A string of bits as specified in Section 8.6.7.

3. personalization_string: The personalization string received from the consuming application. Note that the length of the personalization_string may be zero.

4. security_strength: The security strength for the instantiation. This parameter is optional for HMAC_DRBG, since it is not used.

\section{Output:}

1. initial_working_state: The initial values for $V$, Key and reseed_counter (see Section 10.1.2.1).

\section{HMAC_DRBG Instantiate Process:}

1. seed_material =entropy_input $\|$ nonce $\|$ personalization_string.

2. Key $=0 \times 0000 \ldots 00$ Comment: outlen bits.

3. $V=0 \mathrm{x} 0101 \ldots 01$ Comment: outlen bits.

Comment: Update Key and $V$.

4. $($ Key, $V)=$ HMAC_DRBG_Update (seed_material, Key, $V$ ).

5. reseed_counter $=1$.

6. Return $(V$, Key.reseed_counter $)$. 


\subsubsection{Reseeding an HMAC_DRBG Instantiation}

Notes for the reseed function specified in Section 9.2:

The reseeding of an HMAC_DRBG instantiation requires a call to the

Reseed_function specified in Section 9.2. Process step 6 of that function calls the reseed algorithm specified in this section. The values for min_length are provided in Table 2 of Section 10.1.

The reseed algorithm:

Let HMAC_DRBG_Update be the function specified in Section 10.1.2.2. The following process or its equivalent shall be used as the reseed algorithm for this DRBG mechanism (see step 6 of the reseed process in Section 9.2):

HMAC_DRBG_Reseed_algorithm (working_state, entropy_input, additional_input):

1. working_state: The current values for $V$, Key and reseed_counter (see Section 10.1.2.1).

2. entropy_input: The string of bits obtained from the randomness source.

3. additional_input: The additional input string received from the consuming application. Note that the length of the additional_input string may be zero.

\section{Output:}

1. new_working_state: The new values for $V$, Key and reseed_counter.

\section{HMAC_DRBG Reseed Process:}

1. seed_material =entropy_input $\|$ additional_input.

2. $($ Key, $V)=$ HMAC_DRBG_Update (seed_material, Key, $V$ ).

3. reseed_counter $=1$.

4. Return (V,Key, reseed_counter).

\subsubsection{Generating Pseudorandom Bits Using HMAC_DRBG}

Notes for the generate function specified in Section 9.3:

The generation of pseudorandom bits using an HMAC_DRBG instantiation requires a call to the Generate_function specified in Section 9.3. Process step 8 of that function calls the generate algorithm specified in this section. The values for

max_number_of_bits_per_request and outlen are provided in Table 2 of Section 10.1.

The generate algorithm:

Let HMAC be the keyed hash function specified in [FIPS 198] using the hash function selected for the DRBG mechanism. The value for reseed_interval is defined in Table 2 of Section 10.1.

The following process or its equivalent shall be used as the generate algorithm for this DRBG mechanism (see step 8 of the generate process in Section 9.3): 
HMAC_DRBG_Generate_algorithm (working_state, requested_number_of_bits, additional_input):

1. working_state: The current values for $V$, Key and reseed_counter (see Section 10.1.2.1).

2. requested_number_of_bits: The number of pseudorandom bits to be returned to the generate function.

3. additional_input: The additional input string received from the consuming application. Note that the length of the additional_input string may be zero.

\section{Output:}

1. status: The status returned from the function. The status will indicate SUCCESS, or indicate that a reseed is required before the requested pseudorandom bits can be generated.

2. returned_bits: The pseudorandom bits to be returned to the generate function.

3. new_working_state: The new values for $V$, Key and reseed_counter.

\section{HMAC_DRBG Generate Process:}

1. If reseed_counter > reseed_interval, then return an indication that a reseed is required.

2. If additional_input $\neq N u l l$, then $(K e y, V)=$

HMAC_DRBG_Update (additional_input, Key, V).

3. temp $=$ Null.

4. While $($ len $($ temp $)<$ requested_number_of_bits $)$ do:

$$
4.1 V=\text { HМАС }(K e y, V) \text {. }
$$

4.2 temp $=$ temp $\| V$.

5. returned_bits $=$ leftmost $($ temp, requested_number_of_bits).

6. $(K e y, V)=$ HMAC_DRBG_Update (additional_input, Key, $V$ ).

7. reseed_counter $=$ reseed_counter +1 .

8. Return (SUCCESS, returned_bits, Key, V, reseed_counter). 


\subsection{DRBG Mechanism Based on Block Ciphers}

A block cipher DRBG is based on a block cipher algorithm. The block cipher DRBG mechanism specified in this Recommendation has been designed to use any approved block cipher algorithm and may be used by consuming applications requiring various security strengths, providing that the appropriate block cipher algorithm and key length are used, and sufficient entropy is obtained for the seed.

The maximum security strength that can be supported by the DRBG depends on the block cipher and key size used; the security strengths that can be supported by the block ciphers and key sizes are provided in [SP 800-57].

\subsubsection{CTR_DRBG}

CTR_DRBG uses an approved block cipher algorithm in the counter mode as specified in [SP 800-38A], but allows the counter field to be a subset of the input block, as specified in [SP 80038D]. Note that for TDEA, the input and output block lengths are 64 bits, and for AES, the lengths are 128 bits.

The same block cipher algorithm and key length shall be used for all block cipher operations of this

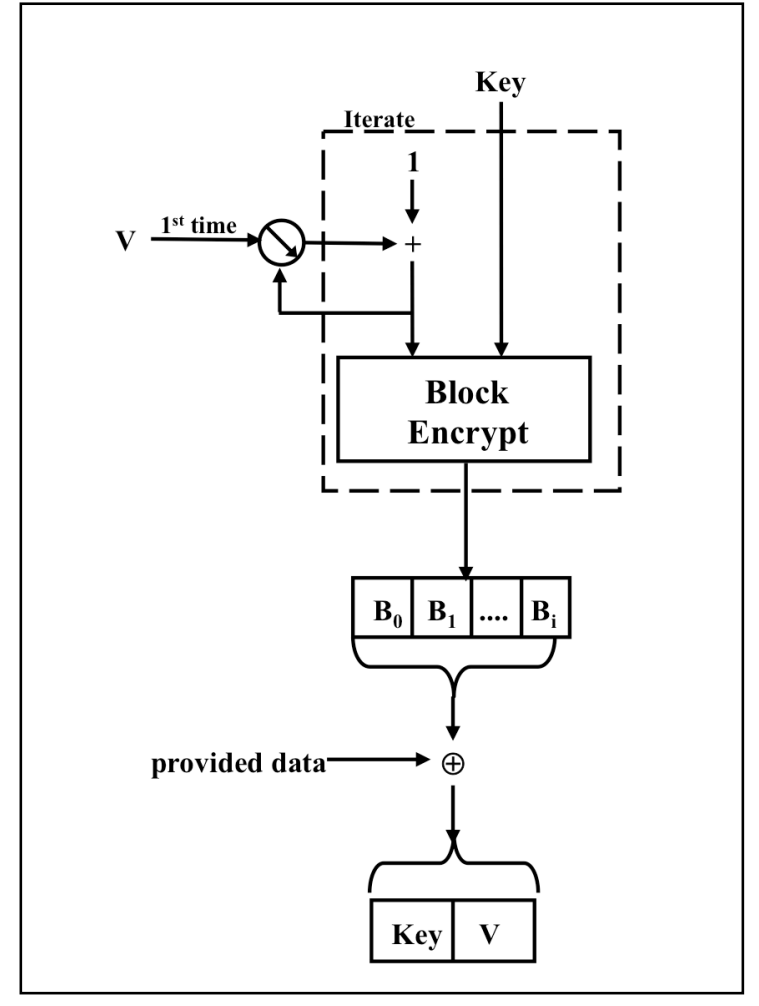

Figure 11: CTR_DRBG Update Function DRBG. The block cipher algorithm and key length shall meet or exceed the security requirements of the consuming application.

CTR_DRBG is specified using an internal function (CTR_DRBG_Update). Figure 11 depicts the CTR_DRBG_Update function. This function is called by the instantiate, generate and reseed algorithms to adjust the internal state when new entropy or additional input is provided, as well as to update the internal state after pseudorandom bits are generated. Figure 12 depicts the CTR_DRBG in three stages. The operations in the top portion of the figure are only performed if the additional input is not null.

Table 3 specifies the values that shall be used for the function envelopes and CTR_DRBG mechanism. 
Table 3: Definitions for the CTR_DRBG

\begin{tabular}{|c|c|c|c|c|}
\hline & 3 Key TDEA & $\begin{array}{c}\text { AES- } \\
128\end{array}$ & $\begin{array}{c}\text { AES- } \\
192\end{array}$ & $\begin{array}{c}\text { AES- } \\
256\end{array}$ \\
\hline Supported security strengths & \multicolumn{4}{|c|}{ See [SP 800-57] } \\
\hline highest_supported_security_strength & \multicolumn{4}{|c|}{ See [SP 800-57] } \\
\hline $\begin{array}{l}\text { Input and output block length } \\
\text { (blocklen) }\end{array}$ & 64 & 128 & 128 & 128 \\
\hline Counter field length (ctr_len) & \multicolumn{4}{|c|}{$4 \leq$ ctr_len $\leq$ blocklen } \\
\hline Key length (keylen) & 168 & 128 & 192 & 256 \\
\hline $\begin{array}{l}\text { Required minimum entropy for } \\
\text { instantiate and reseed }\end{array}$ & \multicolumn{4}{|c|}{ security_strength } \\
\hline Seed length $($ seedlen $=$ outlen + keylen $)$ & 232 & 256 & 320 & 384 \\
\hline \multicolumn{5}{|l|}{ If a derivation function is used: } \\
\hline $\begin{array}{l}\text { Minimum entropy input length } \\
\text { (min_length) }\end{array}$ & \multicolumn{4}{|c|}{ security_strength } \\
\hline $\begin{array}{l}\text { Maximum entropy input length } \\
\text { (max_length) }\end{array}$ & \multicolumn{4}{|c|}{$2^{35}$ bits } \\
\hline $\begin{array}{l}\text { Maximum personalization string } \\
\text { length } \\
\text { (max_personalization_string_length) }\end{array}$ & \multicolumn{4}{|c|}{$2^{35}$ bits } \\
\hline $\begin{array}{l}\text { Maximum additional_input length } \\
\text { (max_additional_input_length) }\end{array}$ & \multicolumn{4}{|c|}{$2^{35}$ bits } \\
\hline \multicolumn{5}{|l|}{ If a derivation function is not used: } \\
\hline $\begin{array}{l}\text { Minimum entropy input length } \\
(\text { min_length }=\text { blocklen }+ \text { keylen })\end{array}$ & \multicolumn{4}{|c|}{ seedlen } \\
\hline $\begin{array}{l}\text { Maximum entropy input length } \\
(\text { max_length }=\text { blocklen }+ \text { keylen })\end{array}$ & \multicolumn{4}{|c|}{ seedlen } \\
\hline $\begin{array}{l}\text { Maximum personalization string } \\
\text { length } \\
\text { (max_personalization_string_length) }\end{array}$ & \multicolumn{4}{|c|}{ seedlen } \\
\hline $\begin{array}{l}\text { Maximum additional_input length } \\
\text { (max_additional_input_length) }\end{array}$ & \multicolumn{4}{|c|}{ seedlen } \\
\hline 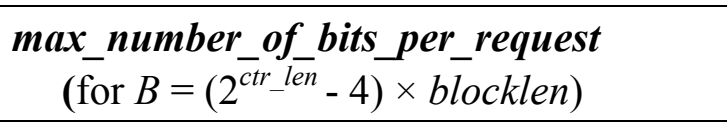 & $\min \left(B, 2^{13}\right)$ & \multicolumn{3}{|c|}{$\min \left(B, 2^{19}\right)$} \\
\hline $\begin{array}{l}\text { Maximum number of requests between } \\
\text { reseeds (reseed_interval) }\end{array}$ & $2^{32}$ & \multicolumn{3}{|c|}{$2^{48}$} \\
\hline
\end{tabular}

Note that the claimed security strength for CTR_DRBG depends on limiting the total number of generate requests and the bits provided per generate request according to the table above. 
Without these limits, it becomes possible, in principle, for an attacker to observe enough outputs from

CTR_DRBG to distinguish its outputs from ideal random bits.

The CTR_DRBG may be implemented to use the block cipher derivation function specified in Section 10.3.2 during instantiation and reseeding. However, the DRBG mechanism is specified to allow an implementation tradeoff with respect to the use of this derivation function. The use of the derivation function is optional if either an approved RBG or an entropy source provides full entropy output when entropy input is requested by the DRBG mechanism. Otherwise, the derivation function shall be used.

Table 3 provides the lengths required for the entropy_input,

personalization_string and additional_input for each case.

When using TDEA as the selected block cipher algorithm, the keys shall be handled as 64-bit blocks containing 56 bits of key and 8 bits of parity as specified for the TDEA engine specified in [SP 800-67].

\subsubsection{CTR_DRBG Internal State}

The internal state for the CTR_DRBG consists of:

1. The working_state:

a. The value $V$ of blocklen bits, which is updated each

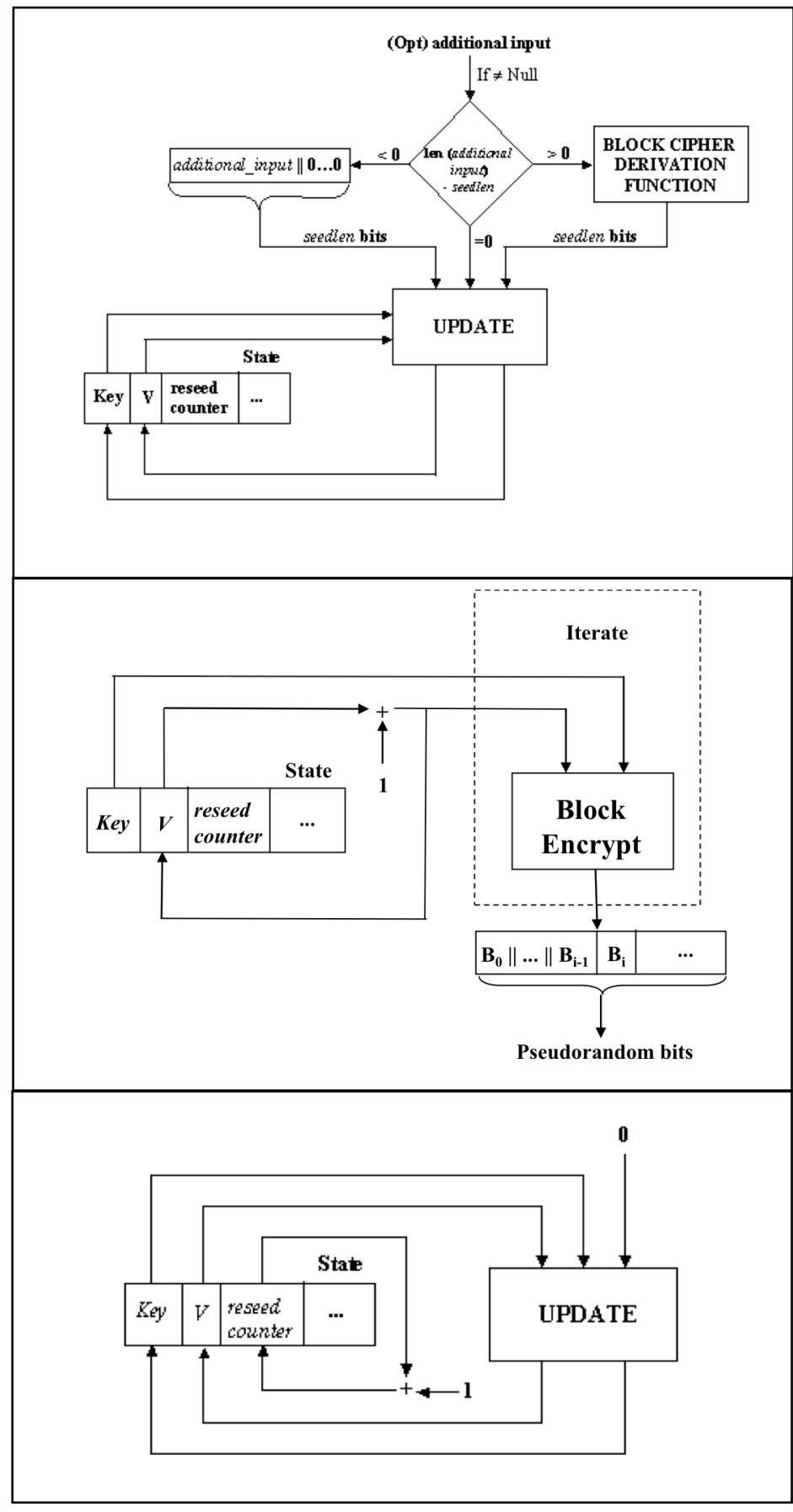

Figure 12: CTR-DRBG time another blocklen bits of output are produced.

b. The keylen-bit Key, which is updated whenever a predetermined number of output blocks are generated.

c. A counter (reseed_counter) that indicates the number of requests for pseudorandom bits since instantiation or reseeding.

2. Administrative information: 
a. The security_strength of the DRBG instantiation.

b. A prediction_resistance_flag that indicates whether or not a prediction resistance capability is required for the DRBG instantiation.

The values of $V$ and Key are the critical values of the internal state upon which the security of this DRBG mechanism depends (i.e., $V$ and Key are the "secret values" of the internal state).

\subsubsection{The Update Function (CTR_DRBG_Update)}

The CTR_DRBG_Update function updates the internal state of the CTR_DRBG using the provided_data. The values for blocklen, keylen and seedlen are provided in Table 3 of Section 10.2.1. The value of ctr_len is known by an implementation. The block cipher operation in step 2.2 of the CTR_DRBG_UPDATE process uses the selected block cipher algorithm. The specification of Block_Encrypt is discussed in Section 10.3.3.

The following or an equivalent process shall be used as the CTR_DRBG_Update function.

CTR_DRBG_Update (provided_data, Key, V):

1. provided_data: The data to be used. This must be exactly seedlen bits in length; this length is guaranteed by the construction of the provided_data in the instantiate, reseed and generate functions.

2. Key: The current value of Key.

3. $V$ : The current value of $V$.

\section{Output:}

1. $K$ : The new value for Key.

2. $V$ : The new value for $V$.

\section{CTR_DRBG_Update Process:}

1. temp $=$ Null.

2. While $($ len $($ temp $)<$ seedlen $)$ do

\subsection{If ctr len $<$ blocklen}

2.1.1 inc $=($ rightmost $(V, c t r$ len $)+1) \bmod 2^{\text {ctr len }}$.

2.1.2 $V=$ leftmost $(V$, blocklen-ctr_len $) \|$ inc.

Else $V=(V+1) \bmod 2^{\text {blocklen }}$.

2.2 output_block $=$ Block_Encrypt $(K e y, V)$.

2.3 temp $=$ temp $\|$ output_block.

3. temp $=$ leftmost (temp, seedlen).

4 temp $=$ temp $\oplus$ provided_data.

5. Key $=$ leftmost $($ temp, keylen $)$.

6. $V=$ rightmost (temp, blocklen). 
7. Return $(K e y, V)$.

\subsubsection{Instantiation of CTR_DRBG}

Notes for the instantiate function specified in Section 9.1:

The instantiation of CTR_DRBG requires a call to the Instantiate_function specified in Section 9.1. Process step 9 of that function calls the instantiate algorithm specified in this section. The values of highest_supported_security_strength and min_length are provided in Table 3 of Section 10.2.1. The contents of the internal state are provided in Section 10.2.1.1.

The instantiate algorithm:

For this DRBG mechanism, there are two cases for processing. In each case, let CTR_DRBG_Update be the function specified in Section 10.2.1.2. The output block length (blocklen), key length (keylen), seed length (seedlen) and security_strengths for the block cipher algorithms are provided in Table 3 of Section 10.2.1.

\subsection{Instantiation When a Derivation Function is Not Used}

When instantiation is performed using this method, full-entropy input is required, and a nonce is not used. The following process or its equivalent shall be used as the instantiate algorithm for this DRBG mechanism:

CTR_DRBG_Instantiate_algorithm (entropy_input, personalization_string, security_strength):

1. entropy_input: The string of bits obtained from the randomness source.

2. personalization_string: The personalization string received from the consuming application. Note that the length of the personalization_string may be zero.

3. security_strength: The security strength for the instantiation. This parameter is optional for CTR_DRBG.

\section{Output:}

1. initial_working_state: The initial values for $V$, Key, and reseed_counter (see Section 10.2.1.1).

\section{CTR_DRBG Instantiate Process:}

1. temp $=$ len (personalization_string).

Comment: Ensure that the length of the personalization_string is exactly seedlen bits. Note that in Section 9.1, processing step 3 obtained an entropy_input of seedlen bits using Table 3 to define the minimum and maximum lengths, which are both equal to seedlen bits.

2. If (temp $<$ seedlen $)$, then personalization_string $=$ personalization_string $\| 0^{\text {seedlen - }}$
temp

3. seed_material $=$ entropy_input $\oplus$ personalization_string. 
4. Key $=0^{\text {keylen }}$ Comment: keylen bits of zeros.

5. $V=0^{\text {blocklen }}$. Comment: blocklen bits of zeros.

6. $(K e y, V)=$ CTR_DRBG_Update (seed_material, Key, V).

7. reseed_counter $=1$.

8. Return (V,Key, reseed_counter $)$.

\subsection{Instantiation When a Derivation Function is Used}

When instantiation is performed using this method, the entropy input may or may not have full entropy; in either case, a nonce is required.

Let $\mathbf{d f}$ be the derivation function specified in Section 10.3.2. When instantiation is performed using this method, a nonce is required, whereas using the method in Section 10.2.1.3.1 does not require a nonce, since full entropy is provided when using that method.

The following process or its equivalent shall be used as the instantiate algorithm for this DRBG mechanism:

CTR_DRBG_Instantiate_algorithm (entropy_input, nonce, personalization_string, security_strength):

1. entropy_input: The string of bits obtained from the randomness source.

2. nonce: A string of bits as specified in Section 8.6.7.

3. personalization_string: The personalization string received from the consuming application. Note that the length of the personalization_string may be zero.

4. security_strength: The security strength for the instantiation. This parameter is optional for CTR_DRBG, since it is not used.

\section{Output:}

1. initial_working_state: The initial values for $V$, Key, and reseed_counter (see Section 10.2.1.1).

\section{CTR_DRBG Instantiate Process:}

1. seed_material $=$ entropy_input $\|$ nonce $\|$ personalization_string.

Comment: Ensure that the length of the seed_material is exactly seedlen bits.

2. $\quad$ seed_material $=\mathbf{d f}($ seed_material, seedlen $)$.

3. Key $=0^{\text {keylen }}$ Comment: keylen bits of zeros.

4. $V=0^{\text {blocklen }}$. Comment: blocklen bits of zeros.

5. $($ Key, $V)=$ CTR_DRBG_Update (seed_material, Key, $V)$.

6. reseed_counter $=1$.

7. Return $(V$, Key, reseed_counter $)$. 


\subsubsection{Reseeding a CTR_DRBG Instantiation}

Notes for the reseed function specified in Section 9.2:

The reseeding of a CTR_DRBG instantiation requires a call to the Reseed_function specified in Section 9.2. Process step 6 of that function calls the reseed algorithm specified in this section. The values for min length are provided in Table 3 of Section 10.2.1.

The reseed algorithm:

For this DRBG mechanism, there are two cases for processing. In each case, let CTR_DRBG_Update be the function specified in Section 10.2.1.2. The seed length (seedlen) is provided in Table 3 of Section 10.2.1.

\subsubsection{Reseeding When a Derivation Function is Not Used}

When reseeding is performed using this method, full-entropy input is required.

The following process or its equivalent shall be used as the reseed algorithm for this DRBG mechanism (see step 6 of the reseed process in Section 9.2):

CTR_DRBG_Reseed_algorithm (working_state, entropy_input, additional_input):

1. working_state: The current values for $V$, Key and reseed_counter (see Section 10.2.1.1).

2. entropy_input: The string of bits obtained from the randomness source.

3. additional_input: The additional input string received from the consuming application. Note that the length of the additional_input string may be zero.

\section{Output:}

1. new_working_state: The new values for $V$, Key, and reseed_counter.

\section{CTR_DRBG Reseed Process:}

1. temp $=$ len (additional_input).

Comment: Ensure that the length of the additional_input is exactly seedlen bits. The maximum length was checked in Section 9.2, processing step 2 , using Table 3 to define the maximum length.

2. If (temp $<$ seedlen $)$, then additional_input $=$ additional_input $\| 0^{\text {seedlen - temp }}$.

3. seed_material $=$ entropy_input $\oplus$ additional_input.

4. $($ Key, $V)=$ CTR_DRBG_Update (seed_material, Key, V).

5. reseed_counter $=1$.

6. Return $(V$, Key, reseed_counter $)$.

\subsection{Reseeding When a Derivation Function is Used}

Let $\mathbf{d f}$ be the derivation function specified in Section 10.3.2. 
The following process or its equivalent shall be used as the reseed algorithm for this DRBG mechanism (see reseed process step 6 of Section 9.2):

CTR_DRBG_Reseed_algorithm (working_state, entropy_input, additional_input)

1. working_state: The current values for $V$, Key and reseed_counter (see Section 10.2.1.1).

2. entropy_input: The string of bits obtained from the randomness source.

3. additional_input: The additional input string received from the consuming application. Note that the length of the additional_input string may be zero.

\section{Output:}

1. new_working_state: The new values for $V$, Key, and reseed_counter.

\section{CTR_DRBG Reseed Process:}

1. seed_material $=$ entropy_input $\|$ additional_input.

Comment: Ensure that the length of the seed_material is exactly seedlen bits.

2. seed_material $=\mathbf{d f}$ (seed_material, seedlen).

3. $($ Key, $V)=$ CTR_DRBG_Update (seed_material, Key, $V$ ).

4. reseed_counter $=1$.

5. Return $(V$, Key, reseed_counter $)$.

\subsubsection{Generating Pseudorandom Bits Using CTR_DRBG}

Notes for the generate function specified in Section 9.3:

The generation of pseudorandom bits using a CTR_DRBG instantiation requires a call to the Generate_function specified in Section 9.3. Process step 8 of that function calls the generate algorithm specified in this section. The values for max_number_of_bits_per_request and max_additional_input_length, and blocklen are provided in Table $\overline{3}$ of Section 10.2.1. If the derivation function is not used, then the maximum allowed length of additional_input $=$ seedlen.

For this DRBG mechanism, there are two cases for the processing. For each case, let CTR_DRBG_Update be the function specified in Section 10.2.1.2, and let Block_Encrypt be the function specified in Section 10.3.3. The seed length (seedlen) and the value of reseed_interval are provided in Table 3 of Section 10.2.1. The value of ctr_len is known by an implementation.

\subsection{Generating Pseudorandom Bits When a Derivation Function is Not Used}

This method of generating bits is used when a derivation function is not used by an implementation.

The following process or its equivalent shall be used as the generate algorithm for this DRBG mechanism (see step 8 of the generate process in Section 9.3.3): 
CTR_DRBG_Generate_algorithm (working_state, requested_number_of_bits, additional_input):

1. working_state: The current values for $V, K e y$, and reseed_counter (see Section 10.2.1.1).

2. requested_number_of_bits: The number of pseudorandom bits to be returned to the generate function.

3. additional_input: The additional input string received from the consuming application. Note that the length of the additional_input string may be zero.

\section{Output:}

1. status: The status returned from the function. The status will indicate SUCCESS, or indicate that a reseed is required before the requested pseudorandom bits can be generated.

2. returned_bits: The pseudorandom bits returned to the generate function.

3. working_state: The new values for $V, K e y$, and reseed_counter.

\section{CTR_DRBG Generate Process:}

1. If reseed_counter > reseed_interval, then return an indication that a reseed is required.

2. If (additional_input $\neq N u l l)$, then

Comment: Ensure that the length of the additional_input is exactly seedlen bits. The maximum length was checked in Section 9.3.3, processing step 4, using Table 3 to define the maximum length. If the length of the additional input is $<$ seedlen, pad with zero bits.

\section{1 temp = len (additional_input).}

2.2 If (temp $<$ seedlen $)$, then additional_input $=$ additional_input $\| 0^{\text {seedlen - temp }}$.

\section{$2.3($ Key, $V)=$ CTR_DRBG_Update (additional_input, Key, V).}

Else additional_input $=0^{\text {seedlen }}$.

3. temp $=$ Null.

4. While $(\operatorname{len}($ temp $)<$ requested_number_of_bits $)$ do:

4.1 If ctr_len $<$ blocklen

4.1.1 inc $=($ rightmost $(V$, ctr_len $)+1) \bmod 2^{\text {ctr_len }}$.

4.1.2 $V=$ leftmost $(V$, blocklen-ctr_len $) \|$ inc.

Else $V=(V+1) \bmod 2^{\text {blocklen }}$. 
4.2 output_block $=$ Block_Encrypt $($ Key, V) .

4.3 temp $=$ temp $\|$ output_block.

5. returned_bits $=$ leftmost $($ temp, requested_number_of_bits).

Comment: Update for backtracking resistance.

6. $(K e y, V)=$ CTR_DRBG_Update (additional_input, Key, $V$ ).

7. reseed_counter $=$ reseed_counter +1 .

8. Return (SUCCESS, returned_bits, Key, V, reseed_counter).

\subsection{Generating Pseudorandom Bits When a Derivation Function is Used}

This method of generating bits is used when a derivation function is used by an implementation.

Let $\mathbf{d f}$ be the derivation function specified in Section 10.3.2.

The following process or its equivalent shall be used as the generate algorithm for this DRBG mechanism (see step 8 of the generate process in Section 9.3.3):

CTR_DRBG_Generate_algorithm (working_state, requested_number_of_bits, additional_input):

1. working_state: The current values for $V, K e y$, and reseed_counter (see Section 10.2.1.1).

2. requested_number_of_bits: The number of pseudorandom bits to be returned to the generate function.

3. additional_input: The additional input string received from the consuming application. Note that the length of the additional_input string may be zero.

\section{Output:}

1. status: The status returned from the function. The status will indicate SUCCESS, or indicate that a reseed is required before the requested pseudorandom bits can be generated.

2. returned_bits: The pseudorandom bits returned to the generate function.

3. working_state: The new values for $V, K e y$, and reseed_counter.

\section{CTR_DRBG Generate Process:}

1. If reseed_counter > reseed_interval, then return an indication that a reseed is required.

2. If (additional_input $\neq N u l l)$, then

2.1 additional_input $=$ Block_Cipher_df (additional_input, seedlen).

$2.2($ Key, $V)=$ CTR_DRBG_Update (additional_input, Key, $V$ ).

Else additional_input $=0^{\text {seedlen }}$.

3. temp $=$ Null. 
4. While $(\operatorname{len}($ temp $)<$ requested_number_of_bits $)$ do:

4.1 If ctr_len $<$ blocklen

4.1.1 inc $=($ rightmost $(V$, ctr_len $)+1) \bmod 2^{\text {ctr len }}$.

4.1.2 $V=$ leftmost $(V$, blocklen-ctr_len $) \|$ inc.

Else

4.1.2 $V=(V+1) \bmod 2^{\text {blocklen }}$.

4.2 output_block $=$ Block_Encrypt $(K e y, V)$.

4.3 temp $=$ temp $\|$ output_block.

5. returned_bits $=$ leftmost $($ temp, requested_number_of_bits).

Comment: Update for backtracking resistance.

6. $(K e y, V)=$ CTR_DRBG_Update (additional_input, Key, $V)$.

7. reseed_counter $=$ reseed_counter +1 .

8. Return (SUCCESS, returned_bits, Key, V, reseed_counter).

\subsection{Auxiliary Functions}

Derivation functions are internal functions that are used during DRBG instantiation and reseeding to either derive internal state values or to distribute entropy throughout a bitstring. Two methods are provided. One method is based on the use of hash functions (see Section 10.3.1), and the other method is based on the use of block cipher algorithms (see Section 10.3.2). The block cipher derivation function specified in Section 10.3.2 uses a BCC function and a Block_Encrypt call that are discussed in Section 10.3.3.

The presence of these derivation functions in this Recommendation does not implicitly approve these functions for any other application.

\subsubsection{Derivation Function Using a Hash Function (Hash_df)}

This derivation function is used by the Hash_DRBG specified Section 10.1.1. The hash-based derivation function hashes an input string and returns the requested number of bits. Let Hash be the hash function used by the DRBG mechanism, and let outlen be its output length.

The following or an equivalent process shall be used to derive the requested number of bits:

Hash_df (input_string, no_of_bits_to_return):

1. input_string: The string to be hashed.

2. no_of_bits_to_return: The number of bits to be returned by Hash_df. The maximum length (max_number_of_bits) is implementation dependent, but shall be less than or equal to $(255 \times$ outlen $)$. no_of_bits_to_return is represented as a 32-bit integer.

\section{Output:}


1. status: The status returned from Hash_df. The status will indicate SUCCESS or ERROR_FLAG.

2. requested_bits: The result of performing the Hash_df.

\section{Hash_df Process:}

1. temp $=$ the Null string.

2. $l e n=\left\lceil\frac{\text { no_of_bits_to_return }}{\text { outlen }}\right\rceil$.

3. counter $=0 \times 01$.

Comment: An 8-bit binary value representing the integer "1".

4. For $i=1$ to len do

Comment : In step 4.1, no_of_bits_to_return is used as a 32-bit string.

4.1 temp $=$ temp $\|$ Hash (counter $\|$ no_of_bits_to_return $\|$ input_string).

4.2 counter $=$ counter +1 .

5. requested_bits $=$ leftmost $($ temp, no_of_bits_to_return $)$.

6. Return (SUCCESS, requested_bits).

\subsubsection{Derivation Function Using a Block Cipher Algorithm (Block_Cipher_df)}

This derivation function is used by the CTR_DRBG that is specified in Section 10.2. BCC and Block_Encrypt are discussed in Section 10.3.3. Let outlen be its output block length, which is a multiple of eight bits for the approved block cipher algorithms, and let keylen be the key length.

The following or an equivalent process shall be used to derive the requested number of bits.

Block_Cipher_df (input_string, no_of_bits_to_return):

1. input_string: The string to be operated on. This string shall be a multiple of eight bits.

2. no_of_bits_to_return: The number of bits to be returned by Block_Cipher_df. The maximum length (max_number_of_bits) is 512 bits for the currently approved block cipher algorithms.

\section{Output:}

1. status: The status returned from Block_Cipher_df. The status will indicate SUCCESS or ERROR_FLAG.

2. requested_bits: The result of performing the Block_Cipher_df.

\section{Block_Cipher_df Process:}

1. If (number_of_bits_to_return $>$ max_number_of_bits), then return an ERROR_FLAG and a Null string. 
2. $L=$ len (input_string) $/ 8$.

3. $N=$ number_of_bits_to_return/8.
Comment: $L$ is the bitstring representation of the integer resulting from len (input_string)/8. $L$ shall be represented as a 32-bit integer.

Comment: $N$ is the bitstring representation of the integer resulting from number_of_bits_to_return/8. $N$ shall be represented as a 32-bit integer.

Comment: Prepend the string length and the requested length of the output to the input_string.

4. $S=L\|N\|$ input_string $\| 0 \times 80$.

Comment: Pad $S$ with zeros, if necessary.

5. While (len $(S) \bmod$ outlen $) \neq 0$, do

$$
S=S \| 0 \times 00 \text {. }
$$

Comment: Compute the starting value.

6. temp $=$ the Null string.

7. $i=0$.

Comment: $i$ shall be represented as a 32-bit integer, i.e., len $(i)=32$.

8. $K=$ leftmost $(0 x 00010203 \ldots 1 \mathrm{D} 1 \mathrm{E} 1 \mathrm{~F}$, keylen $)$.

9. While len $($ temp $)<$ keylen + outlen, do
9.1 IV $=i \| 0^{\text {outlen }-\operatorname{len}(i)}$.
Comment: The 32-bit integer representation of $i$ is padded with zeros to outlen bits.

9.2 temp $=$ temp $\| \mathbf{B C C}(K,(I V \| S))$.

$9.3 i=i+1$.

Comment: Compute the requested number of bits.

10. $K=$ leftmost (temp, keylen).

11. $X=$ select (temp, keylen+1, keylen+outlen).

12. temp $=$ the Null string.

13. While len $($ temp $)<$ number_of_bits_to_return, do

$13.1 X=$ Block_Encrypt $(K, X)$.

13.2 temp $=$ temp $\| X$.

14. requested_bits $=$ leftmost $($ temp, number_of_bits_to_return) .

15. Return (SUCCESS, requested_bits).

\subsubsection{BCC and Block_Encrypt}

Block_Encrypt is used for convenience in the specification of the BCC function. This function is not specifically defined in this Recommendation, but has the following meaning: 
Block_Encrypt: A basic encryption operation that uses the selected block cipher algorithm. The function call is:

$$
\text { output_block }=\text { Block_Encrypt }(\text { Key, input_block })
$$

For TDEA, the basic encryption operation is called the forward cipher operation (see [SP 800-67]); for AES, the basic encryption operation is called the cipher operation (see [FIPS 197]). The basic encryption operation is equivalent to an encryption operation on a single block of data using the ECB mode.

For the BCC function, let outlen be the length of the output block of the block cipher algorithm to be used.

The following or an equivalent process shall be used to derive the requested number of bits.

$\mathbf{B C C}($ Key, data):

1. Key: The key to be used for the block cipher operation.

2. data: The data to be operated upon. Note that the length of data must be a multiple of outlen. This is guaranteed by Block_Cipher_df process steps 4 and 8.1 in Section 10.3.2.

\section{Output:}

1. output_block: The result to be returned from the BCC operation.

\section{BCC Process:}

1. chaining_value $=0^{\text {outlen }}$. Comment: Set the first chaining value to outlen zeros.

2. $n=$ len $($ data $) / o u t l e n$.

3. Starting with the leftmost bits of data, split data into $n$ blocks of outlen bits each, forming block $_{1}$ to block $_{n}$.

4. For $i=1$ to $n$ do

4.1 input_block $=$ chaining_value $\oplus$ block $_{i}$.

4.2 chaining_value $=$ Block_Encrypt $($ Key, input_block $)$.

5. output_block=chaining_value.

6. Return (output_block). 


\section{Assurance}

A user of a DRBG employed for cryptographic purposes requires assurance that the generator actually produces (pseudo) random and unpredictable bits. The user needs assurance that the design of the generator, its implementation and its use to support cryptographic services are adequate to protect the user's information. In addition, the user requires assurance that the generator continues to operate correctly.

The design of each DRBG mechanism in this Recommendation has received an evaluation of its security properties prior to its selection for inclusion in this Recommendation.

An implementer selects a DRBG mechanism (e.g., HMAC_DRBG), an appropriate cryptographic primitive (e.g., SHA-256 or SHA-512), the DRBG functions to be used (i.e., instantiate, generate and/or reseed), and will determine whether or not the DRBG will be distributed (see Section 8.5). Each choice of components effectively defines a different DRBG type. For example, an implementation of HMAC_DRBG using SHA-256 is considered to be a different DRBG than HMAC_DRBG using SHA-512.

An implementation shall be validated for conformance to this Recommendation by a NVLAPaccredited laboratory (see Section 11.2). Such validations provide a higher level of assurance that the DRBG mechanism is correctly implemented.

Health tests on the DRBG mechanism shall be implemented within a DRBG mechanism boundary or sub-boundary in order to determine that the process continues to operate as designed and implemented. See Section 11.3 for further information.

A cryptographic module containing a DRBG mechanism shall be validated (see [FIPS 140]). The consuming application or cryptographic service that uses a DRBG mechanism should also be validated and periodically tested for continued correct operation. However, this level of testing is outside the scope of this Recommendation.

Note that any entropy input used for testing (either for validation testing or health testing) may be publicly known. Therefore, entropy input used for testing shall not be used for normal operational use.

\subsection{Minimal Documentation Requirements}

A set of documentation shall be developed that will provide assurance to users and validators that the DRBG mechanisms in this Recommendation have been implemented properly. Much of this documentation could be placed in a user manual. This documentation shall consist of the following as a minimum:

- Document the method for obtaining entropy input.

- Document how the implementation has been designed to permit implementation validation and health testing.

- Document the type of DRBG mechanism (e.g., CTR_DRBG), and the cryptographic primitives used (e.g., AES-128 or SHA-256).

- Document the security strengths supported by the implementation. 
- Document features supported by the implementation (e.g., prediction resistance, personalization string, additional input, etc.).

- If DRBG mechanism functions are distributed, specify the mechanisms that are used to protect the confidentiality and integrity of the internal state or parts of the internal state that are transferred between the distributed DRBG mechanism sub-boundaries (i.e., provide documentation about the secure channel).

- In the case of the CTR_DRBG, indicate whether a derivation function is provided. If a derivation function is not used, document that the implementation can only be used when full entropy input is available.

- Document any support functions other than health testing.

- If periodic testing is performed for the generate function, document the intervals and provide a justification for the selected intervals (see Section 11.3.3).

- Document whether the DRBG functions can be tested on demand.

- Document how the integrity of the health tests will be determined subsequent to implementation validation testing.

\subsection{Implementation Validation Testing}

A DRBG mechanism shall be tested for conformance to this Recommendation. A DRBG mechanism shall be designed to be tested to ensure that the product is correctly implemented. A testing interface shall be available for this purpose in order to allow the insertion of input and the extraction of output for testing.

Implementations to be validated shall include the following:

- The documentation specified in Section 11.1.

- Any documentation or results required in derived test requirements.

All DRBG functions included in an implementation shall be tested, including the health test functionality. The error handling of all implemented DRBG functions will be tested. See Section 11.4 for expected error handling behavior.

Note that when the uninstantiate function is tested, testing shall demonstrate that the internal state has been zeroized.

\subsection{Health Testing}

A DRBG implementation shall perform self-tests to obtain assurance that the DRBG continues to operate as designed and implemented (health testing). The testing function(s) within a DRBG mechanism boundary (or sub-boundary) shall test each DRBG mechanism function within that boundary (or sub-boundary), with the possible exception of the health test function itself. A DRBG implementation may optionally perform other self-tests for DRBG functionality in addition to the tests specified in this Recommendation. 
The testing of the error handling capability is not required during the conduct of health tests. However, errors encountered during health testing shall be handled as discussed in Section 11.4.2.

All data output from the DRBG mechanism boundary (or sub-boundary) shall be inhibited while these tests are performed. The results from known-answer-tests (see Section 11.3.1) shall not be output as random bits during normal operation.

\subsubsection{Known Answer Testing}

Known-answer testing shall be conducted as specified below. A known-answer test involves operating the DRBG mechanism with data for which the correct output is already known, and determining if the calculated output equals the expected output (the known answer). The test fails if the calculated output does not equal the known answer. In this case, the DRBG mechanism shall enter an error state and output an error indicator (see Section 11.4).

Generalized known-answer testing is specified in Sections 11.3.2 through 11.3.5. With the possible exception of the health test function itself, testing shall be performed on all implemented DRBG mechanism functions within a DRBG boundary (if all functions are in the same device) or sub-boundary (if functions are distributed) (see Section 8.5). Documentation shall be provided that addresses the continued integrity of the health tests (see Section 11.1).

Known-answer tests shall be conducted on each DRBG function within a boundary or subboundary prior to the first use of that DRBG (e.g., during the power-on self-testing sequence).

\subsubsection{Testing the Instantiate Function}

Known-answer tests on the instantiate function shall use a security strength that will be available during normal operations. If prediction resistance has been implemented, the prediction_resistance_flag shall also be used. A representative fixed value and length of the entropy_input, nonce and personalization_string (if supported) shall be used; the value of the entropy_input used during testing shall not be intentionally reused during normal operations (either by the instantiate or the reseed functions).

If the values used during the test produce the expected results, then the instantiate function may be used during normal operation.

An implementation should provide a capability to test the instantiate function on demand.

\subsubsection{Testing the Generate Function}

During generate-function testing, a representative fixed value and length for the requested_number_of_bits and additional_input (if supported) shall be used. If prediction resistance is supported, then the use of the prediction_resistance_request parameter shall be tested.

If the values used during the test produce the expected results, then the generate function may be used during normal operation.

Bits generated during health testing shall not be output as pseudorandom bits.

An implementation should provide a capability to test the generate function on demand. 
In addition to testing the generate function before first use (see Section 11.3.1), known-answer tests should be performed at reasonable intervals, as defined by the implementer.

\subsubsection{Testing the Reseed Function}

Known-answer testing of the reseed function shall use the security_strength in the internal state of the (testing) instantiation to be reseeded. A representative value of the entropy_input and additional_input (if supported) shall be used (see Sections 8.3 and 10). If prediction resistance for the reseed function is supported, then the use of the prediction_resistance_request parameter shall be tested.

If the values used during the test produce the expected results, then the reseed function may be used during normal operation.

An implementation should provide a capability to test the reseed function on demand.

\subsubsection{Testing the Uninstantiate Function}

Testing of the uninstantiate function is not required during health testing.

\subsection{Error Handling}

The expected errors are indicated for each DRBG mechanism function (see Sections 9.1 through 9.4) and for the derivation functions in Section 10.3. The error handling routines should indicate the type of error.

\subsubsection{Errors Encountered During Normal Operation}

Many errors that occur during normal operation may be caused by a consuming application's improper DRBG request or possibly the current unavailability of entropy; these errors are indicated by "ERROR_FLAG" in the pseudocode. In these cases, the consuming application user is responsible for correcting the request within the limits of the user's organizational security policy. For example, if a failure indicating an invalid requested security strength is returned, a security strength higher than the DRBG or the DRBG instantiation can support has been requested. The user may reduce the requested security strength if the organization's security policy allows the information to be protected using a lower security strength, or the user shall use an appropriately instantiated DRBG.

Catastrophic errors (i.e., errors indicated by the CATASTROPHIC_ERROR_FLAG in the pseudocode) detected during normal operation shall be treated in the same manner as an error detected during health testing (see Section 11.4.2).

\subsubsection{Errors Encountered During Health Testing}

Errors detected during health testing shall be perceived as catastrophic DRBG failures.

When a DRBG fails a health test or a catastrophic error is detected during normal operation, the DRBG shall enter an error state and output an error indicator. The DRBG shall not perform any instantiate, generate or reseed operations while in the error state, and pseudorandom bits shall not be output when an error state exists. When in an error state, user intervention (e.g., power 
cycling of the DRBG) shall be required to exit the error state, and the DRBG shall be reinstantiated before the DRBG can be used to produce pseudorandom bits. Examples of such errors include:

- A test deliberately inserts an error, and the error is not detected, or

- A result is returned from the instantiate, reseed or generate function that was not expected. 


\section{Appendix A: (Normative) Conversion and Auxiliary Routines}

\section{A.1 Bitstring to an Integer}

Bitstring_to_integer $\left(b_{1}, b_{2}, \ldots, b_{n}\right)$ :

Input:

1. $b_{1}, b_{2}, \ldots, b_{n} \quad$ The bitstring to be converted.

\section{Output:}

1. $x$ The requested integer representation of the bitstring.

\section{Process:}

1. Let $\left(b_{1}, b_{2}, \ldots, b_{n}\right)$ be the bits of a bitstring from leftmost to rightmost (i.e., most significant to least significant).

2. $x=\sum_{i=1}^{n} 2^{(n-i)} b_{i}$

3. Return $(x)$.

In this Recommendation, the binary length of an integer $x$ is defined as the smallest integer $n$ satisfying $x<2^{n}$.

\section{A.2 Integer to a Bitstring}

Integer_to_bitstring $(x)$ :

\section{Input:}

1. $x$ The non-negative integer to be converted.

\section{Output:}

1. $b_{1}, b_{2}, \ldots, b_{n}$ The bitstring representation of the integer $x$.

\section{Process:}

1. Let $\left(b_{1}, b_{2}, \ldots, b_{n}\right)$ represent the bitstring, where $b_{1}=0$ or 1 , and $b_{1}$ is the most significant bit, while $b_{n}$ is the least significant bit.

2. For any integer $n$ that satisfies $x<2^{n}$, the bits $b_{i}$ shall satisfy:

$$
x=\sum_{i=1}^{n} 2^{(n-i)} b_{i} .
$$

3. Return $\left(b_{1}, b_{2}, \ldots, b_{n}\right)$.

In this Recommendation, the binary length of the integer $x$ is defined as the smallest integer $n$ that satisfies $x<2^{n}$. 


\section{A.3 Integer to a Byte String}

Integer_to_byte_string $(x)$ :

Input:

1. A non-negative integer $x$, and the intended length $n$ of the byte string satisfying

$$
2^{8 n}>x \text {. }
$$

\section{Output:}

1. A byte string $B$ of length $n$ bytes.

\section{Process:}

1. Let $B_{1}, B_{2}, \ldots, B_{n}$ be the bytes of $B$ from leftmost to rightmost.

2. The bytes of $B$ shall satisfy:

$$
x=\sum_{i=1}^{n} 2^{8(n-i)} B_{i} .
$$

3. Return $(B)$.

\section{A.4 Byte String to an Integer}

\section{Byte_string_to_integer (B):}

\section{Input:}

1. A byte string $B$ of length $n$ bytes.

\section{Output:}

1. A non-negative integer $x$.

\section{Process:}

1. Let $B_{1}, B_{2}, \ldots, B_{n}$ be the bytes of $B$ from leftmost to rightmost.

2. $x$ is defined as follows:

$$
x=\sum_{i=1}^{n} 2^{8(n-i)} B_{i} .
$$

3. Return $(x)$.

\section{A.5 Converting Random Bits into a Random Number}

In some cryptographic applications, sequences of random numbers are required $\left(a_{0}, a_{1}, a_{2}, \ldots\right)$, where:

i) Each integer $a_{i}$ satisfies $0 \leq a_{i} \leq r$-1, for some positive integer $r$ (the range of the random numbers); 
ii) The equation $a_{i}=s$ holds, with probability almost exactly $1 / r$, for any $i \geq 0$ and for any $s$ $(0 \leq s \leq r-1)$

iii) Each value $a_{i}$ is statistically independent of any set of values $a_{j}(j \neq i)$.

Four techniques are specified for generating sequences of random numbers from sequences of random bits.

If the range of the number required is $a \leq a_{i} \leq b$, rather than $0 \leq a_{i} \leq r-1$, then a random number in the desired range can be obtained by computing $a_{i}+a$, where $a_{i}$ is a random number in the range $0 \leq a_{i} \leq b-a$ (that is, when $r=b-a+1$ ).

\section{A.5.1 The Simple Discard Method}

Let $m$ be the number of bits needed to represent the value $(r-1)$. The following method may be used to generate the random number $a$ :

1. Use the random bit generator to generate a sequence of $m$ random bits, $\left(b_{0}, b_{1}, \ldots, b_{m-1}\right)$.

2. Let $c=\sum_{i=0}^{m-1} 2^{i} b_{i}$.

3. If $c<r$ then put $a=c$, else discard $c$ and go to Step 1 .

This method produces a random number $a$ with no skew (no bias). A possible disadvantage of this method, in general, is that the time needed to generate such a random $a$ is not a fixed length of time because of the conditional loop.

The ratio $r / 2^{m}$ is a measure of the efficiency of the technique, and this ratio will always satisfy $0.5<r / 2^{m} \leq 1$. If $r / 2^{m}$ is close to 1 , then the above method is simple and efficient. However, if $r / 2^{m}$ is close to 0.5 , then the simple discard method is less efficient, and the more complex method below is recommended.

\section{A.5.2 The Complex Discard Method}

Choose a small positive integer $t$ (the number of same-size random number outputs desired), and then let $m$ be the number of bits in $\left(r^{t}-1\right)$. This method may be used to generate a sequence of $t$ random numbers $\left(a_{0}, a_{1}, \ldots, a_{t-1}\right)$ :

1. Use the random bit generator to generate a sequence of $m$ random bits, $\left(b_{0}, b_{1}, \ldots, b_{m-1}\right)$.

2. Let $c=\sum_{i=0}^{m-1} 2^{i} b_{i}$.

3. If $c<r^{t}$, then

let $\left(a_{0}, a_{1}, \ldots, a_{t-1}\right)$ be the unique sequence of values satisfying $0 \leq a_{i} \leq r-1$ such that

$$
c=\sum_{i=0}^{t-1} r^{i} a_{i}
$$

else discard $c$ and go to Step 1 . 
This method produces random numbers $\left(a_{0}, a_{1}, \ldots, a_{t-1}\right)$ with no skew. A possible disadvantage of this method, in general, is that the time needed to generate these numbers is not a fixed length of time because of the conditional loop. The complex discard method may have better overall performance than the simple discard method if many random numbers are needed.

The ratio $r^{t} / 2^{m}$ is a measure of the efficiency of the technique, and this ratio will always satisfy $0.5<r^{t} / 2^{m} \leq 1$. Hence, given $r$, it is recommended to choose $t$ so that $t$ is the smallest value such that $r^{t} / 2^{m}$ is close to 1 . For example, if $r=3$, then choosing $t=3$ means that $m=5$ (as $r^{t}$ is 27) and $r^{t} / m=27 / 32 \approx 0.84$, and choosing $t=5$ means that $m=8$ (as $r^{t}$ is 243 ) and $r^{t} / m=243 / 256 \approx$ 0.95 . The complex discard method coincides with the simple discard method when $t=1$.

\section{A.5.3 The Simple Modular Method}

Let $m$ be the number of bits needed to represent the value $(r-1)$, and let $s$ be a security parameter. The following method may be used to generate one random number $a$ :

1. Use the random bit generator to generate a sequence of $m+s$ random bits, $\left(b_{0}, b_{1}, \ldots, b_{m+s-}\right.$ 1).

2. Let $c=\sum_{i=0}^{m+s-1} 2^{i} b_{i}$.

3. Let $a=c \bmod r$.

The simple modular method can be coded to take constant time. This method produces a random value with a negligible skew, that is, the probability that $a_{i}=w$ for any particular value of $w(0 \leq$ $w \leq r-1$ ) is not exactly $1 / r$. However, for a large enough value of $s$, the difference between the probability that $a_{i}=w$ for any particular value of $w$ and $1 / r$ is negligible. The value of $s$ shall be greater than or equal to 64 . 


\section{Appendix B: (Informative) \\ Example Pseudocode for Each DRBG Mechanism}

The internal states in these examples are considered to be an array of states, identified by state_handle. A particular state is addressed as internal_state (state_handle), where the value of state_handle begins at 0 and ends at $n-1$, and $n$ is the number of internal states provided by an implementation. A particular element in the internal state is addressed by internal_state (state_handle).element. In an empty internal state, all bitstrings are set to Null, and all integers are set to 0 .

For each example in this appendix, arbitrary values have been selected that are consistent with the allowed values for each DRBG mechanism, as specified in the appropriate table in Section 10 .

The pseudocode in this appendix does not include the necessary conversions (e.g., integer to bitstring) for an implementation. When conversions are required, they shall be accomplished as specified in Appendix A.

The following routine is defined for these pseudocode examples:

Find_state_space (): A function that finds an unused internal state. The function returns a status (either "Success" or a message indicating that an unused internal state is not available) and, if status = "Success", a state_handle that points to an available internal_state in the array of internal states. If status $\neq$ "Success", an invalid state_handle is returned.

When the uninstantantiate function is invoked in the following examples, the function specified in Section 9.4 is called.

\section{B.1 Hash_DRBG Example}

This example of Hash_DRBG uses the SHA-1 hash function, and prediction resistance is supported. Both a personalization string and additional input are supported. A 32-bit incrementing counter is used as the nonce for instantiation (instantiation_nonce); the nonce is initialized when the DRBG is instantiated (e.g., by a call to the clock or by setting it to a fixed value) and is incremented for each instantiation.

A total of ten internal states are provided (i.e., ten instantiations may be handled simultaneously).

For this implementation, the functions and algorithms are "inline", i.e., the algorithms are not called as separate routines from the function envelopes. Also, the Get_entropy_input function uses only three input parameters, since the first two parameters (as specified in Section 9) have the same value.

The internal state contains values for $V, C$, reseed_counter, security_strength and prediction_resistance_flag, where $V$ and $\mathrm{C}$ are bitstrings, and reseed_counter, security_strength and the prediction_resistance flag are integers. A requested prediction resistance capability is indicated when prediction_resistance_flag $=1$.

In accordance with Table 2 in Section 10.1, the 112- and 128-bit security strengths may be instantiated. Using SHA-1, the following definitions are applicable for the instantiate, generate and reseed functions and algorithms: 
1. highest_supported_security_strength $=128$.

2. Output block length (outlen) $=160$ bits.

3. Required minimum entropy for instantiation and reseed = security_strength.

4. Seed length $($ seedlen $)=440$ bits.

5. Maximum number of bits per request (max_number_of_bits_per_request $)=5000$ bits.

6. Reseed interval (reseed_interval) $=100000$ requests.

7. Maximum length of the personalization string (max_personalization_string_length) $=$ 512 bits.

8. Maximum length of additional_input (max_additional_input_string_length $)=512$ bits.

9. Maximum length of entropy input $\left(\max \_\right.$length $)=1000$ bits.

\section{B.1.1 Instantiation of Hash_DRBG}

This implementation will return a text message and an invalid state handle (-1) when an error is encountered. Note that the value of instantiation_nonce is an internal value that is always available to the instantiate function.

Note that this implementation does not check the prediction_resistance_flag, since the implementation has been designed to support prediction resistance. However, if a consuming application actually wants prediction resistance, the implementation expects that prediction_resistance flag $=1$ during instantiation; this will be used in the generate function in Appendix B.1.3.

\section{Hash_DRBG_Instantiate_function:}

Input: integer (requested_instantiation_security_strength, prediction_resistance_flag), bitstring personalization_string.

Output: string status, integer state_handle.

\section{Process:}

Comment: Check the input parameters.

1. If (requested_instantiation_security_strength > 128), then Return ("Invalid requested_instantiation_security_strength", -1).

2. If (len (personalization_string) > 512), then Return ("Personalization_string too long", -1).

Comment: Set the security_strength to one of the valid security strengths.

3. If (requested_instantiation_security_strength $\leq 112$ ), then security_strength $=112$ Else security_strength $=128$.

Comment: Get the entropy_input. 
4. (status, entropy_input) $=$ Get_entropy_input (security_strength, 1000, prediction_resistance_request).

5. If (status $\neq$ "Success"), then Return (status, -1 ).

Comment: Increment the nonce; actual coding must ensure that it wraps when the storage limit is reached.

6. instantiation_nonce $=$ instantiation_nonce +1 .

Comment: The instantiate algorithm is provided in steps 7 to 11 .

7. seed_material $=$ entropy_input $\|$ instantiation_nonce $\|$ personalization_string.

8. seed = Hash_df (seed_material, 440). Comment: Hash_df is defined in Section 10.3.1.

9. $V=$ seed.

10. $C=$ Hash_df $((0 \mathrm{x} 00 \| V), 440)$.

11. reseed_counter $=1$.

Comment: Find an unused internal state.

12. $($ status, state_handle $)=$ Find_state_space () .

13. If (status $\neq$ "Success"), then Return (status, -1).

14. Save the internal state.

14.1 internal_state (state_handle). $V=V$.

14.2 internal_state (state_handle).C $=C$.

14.3 internal_state (state_handle).reseed_counter=reseed_counter.

14.4 internal_state (state_handle). security_strength = security_strength.

14.5 internal_state (state_handle).prediction_resistance_flag $=$ prediction_resistance_flag.

15. Return ("Success", state_handle).

\section{B.1.2 Reseeding a Hash_DRBG Instantiation}

The implementation is designed to return a text message as the status when an error is encountered.

\section{Hash_DRBG_Reseed_function:}

Input: integer (state_handle, prediction_resistance_request), bitstring additional_input.

Output: string status.

Process:

Comment: Check the validity of the state_handle. 
1. If $(($ state_handle $<0)$ or $($ state_handle $>9)$ or $($ internal_state $($ state_handle $)=\{$ Null, Null, $0,0,0\})$ ), then Return ("State not available for the state_handle").

Comment: Get the internal state values needed to determine the new internal state.

2. Get the appropriate internal_state values.

$$
\begin{aligned}
& V=\text { internal_state }(\text { state_handle).V. } \\
& \text { security_strength }=\text { internal_state(state_handle).security_strength. } \\
& \text { Check the length of the additional_input. }
\end{aligned}
$$

3. If (Ien (additional_input) > 512), then Return ("additional_input too long").

Comment: Get the entropy_input.

4. (status, entropy_input) $=$ Get_entropy_input (security_strength, 1000, prediction_resistance_request).

5. If (status $\neq$ "Success"), then Return (status).

Comment: The reseed algorithm is provided in steps 6 to 10 .

6. seed_material $=0 \mathrm{x} 01\|V\|$ entropy_input $\|$ additional_input.

7. seed $=$ Hash_df (seed_material, 440).

8. $V=$ seed.

9. $C=$ Hash_df $((0 \mathrm{x} 00 \| V), 440)$.

10. reseed_counter $=1$.

Comment: Update the working_state portion of the internal state.

11. Update the appropriate state values.

11.1 internal_state (state_handle). $V=V$.

11.2 internal_state (state_handle). $C=C$.

11.3 internal_state (state_handle).reseed_counter=reseed_counter.

12. Return ("Success").

\section{B.1.3 Generating Pseudorandom Bits Using Hash_DRBG}

The implementation returns a Null string as the pseudorandom bits if an error has been detected. Prediction resistance is requested when prediction_resistance_request $=1$.

In this implementation, prediction resistance is requested by supplying prediction_resistance_request $=1$ when the Hash_DRBG function is invoked.

\section{Hash_DRBG_Generate_function:}


Input: integer (state_handle, requested_no_of_bits, requested_security_strength, prediction_resistance_request), bitstring additional_input.

Output: string status, bitstring pseudorandom_bits.

Process:

Comment: Check the validity of the state_handle.

1. If $(($ state_handle $<0)$ or (state_handle $>9)$ or (state (state_handle $)=\{$ Null, Null, 0 , $0,0\})$ ), then Return ("State not available for the state_handle", Null).

2. Get the internal state values.

$2.1 V=$ internal_state (state_handle). $V$.

$2.2 C=$ internal_state (state_handle).C.

2.3 reseed_counter $=$ internal_state (state_handle).reseed_counter.

2.4 security_strength $=$ internal_state $($ state_handle).security_strength .

2.5 prediction_resistance_flag $=$ internal_state

(state_handle).prediction_resistance_flag.

Comment: Check the validity of the other input parameters.

3. If (requested_no_of_bits > 5000) then Return ("Too many bits requested", Null).

4. If (requested_security_strength > security_strength), then Return ("Invalid requested_security_strength", Null).

5. If (len (additional_input) > 512), then Return ("additional_input too long", Null).

6. If $(($ reseed_counter $>100000)$ or $($ prediction_resistance_request $=1))$, then

6.1 status $=$ Hash_DRBG_Reseed_function (state_handle, prediction_resistance_request, additional_input).

6.2 If (status $\neq$ "Success"), then Return (status, Null).

6.3 Get the new internal state values that have changed.

7.3.1 V=internal_state (state_handle). $V$.

7.3.2 C = internal_state (state_handle).C.

7.3.3 reseed_counter $=$ internal_state $($ state_handle).reseed_counter .

6.4 additional_input $=$ Null.

Comment: Steps 7 to 15 provide the rest of the generate algorithm. Note that in this implementation, the Hashgen routine specified in Section 10.1.1.4 is provided inline as steps 8 to 12 .

7. If (additional_input $\neq N u l l$ ), then do

$7.1 w=$ Hash $(0 \times 02\|V\|$ additional_input $)$. 
$7.2 V=(V+w) \bmod 2^{440}$.

8. $m=\left\lceil\frac{\text { requested_no_of_bits }}{\text { outlen }}\right\rceil$.

9. $\quad$ data $=V$.

10. $W=$ the Null string.

11. For $i=1$ to $m$

$11.1 w=$ Hash $($ data $)$.

$11.2 W=W \| w$.

11.3 data $=($ data +1$) \bmod 2^{440}$.

12.pseudorandom_bits $=\operatorname{leftmost}(W$,requested_no_of_bits $)$.

13. $H=$ Hash $(0 \mathrm{x} 03 \| V)$.

14. $V=(V+H+C+$ reseed_counter $) \bmod 2^{440}$.

15. reseed_counter $=$ reseed_counter +1 .

Comments: Update the working_state.

16. Update the changed values in the state.

16.1 internal_state (state_handle). $V=V$.

16.2 internal_state (state_handle).reseed_counter $=$ reseed_counter.

17. Return ("Success", pseudorandom_bits).

\section{B.2 HMAC_DRBG Example}

This example of HMAC_DRBG uses the SHA-256 hash function. Reseeding and prediction resistance are not supported. The nonce for instantiation consists of a random value with security_strength/2 bits of entropy; the nonce is obtained by increasing the call for entropy bits via the Get_entropy_input call by security_strength/2 bits (i.e., by adding security_strength/2 bits to the security_strength value). The HMAC_DRBG_Update function is specified in Section 10.1.2.2.

A personalization string is supported, but additional input is not. A total of three internal states are provided. For this implementation, the functions and algorithms are written as separate routines. Also, the Get_entropy_input function uses only two input parameters, since the first two parameters (as specified in Section 9) have the same value, and prediction resistance is not available.

The internal state contains the values for $V$, Key, reseed_counter, and security_strength, where $V$ and $C$ are bitstrings, and reseed_counter and security_strength are integers.

In accordance with Table 2 in Section 10.1, security strengths of 112, 128, 192 and 256 bits may be instantiated. Using SHA-256, the following definitions are applicable for the instantiate and generate functions and algorithms: 
1. highest_supported_security_strength $=256$.

2. Output block $($ outlen $)=256$ bits.

3. Required minimum entropy for the entropy input at instantiation $=(3 / 2)$ security_strength (this includes the entropy required for the nonce).

4. Seed length (seedlen $)=440$ bits.

5. Maximum number of bits per request (max_number_of_bits_per_request $)=7500$ bits.

6. Reseed_interval (reseed_interval) $=10000$ requests.

7. Maximum length of the personalization string (max_personalization_string_length) $=$ 160 bits.

8. Maximum length of the entropy input $\left(\max \_\right.$length $)=1000$ bits.

\section{B.2.1 Instantiation of HMAC_DRBG}

This implementation will return a text message and an invalid state handle $(-1)$ when an error is encountered.

\section{HMAC_DRBG_Instantiate_function:}

Input: integer (requested_instantiation_security_strength), bitstring personalization_string.

Output: string status, integer state_handle.

\section{Process:}

Check the validity of the input parameters.

1. If (requested_instantiation_security_strength > 256), then Return ("Invalid requested_instantiation_security_strength", -1).

2. If (len (personalization_string) > 160), then Return ("Personalization_string too long", -1)

Comment: Set the security_strength to one of the valid security strengths.

3. If (requested_security_strength $\leq 112)$, then security_strength $=112$ Else if (requested_security_strength $\leq 128)$, then security_strength $=128$ Else if (requested_security_strength $\leq 192)$, then security_strength $=192$ Else security_strength $=256$.

Comment: Get the entropy_input and the nonce.

4. min_entropy $=1.5 \times$ security_strength.

5. (status, entropy_input) $=$ Get_entropy_input (min_entropy, 1000).

6. If (status $\neq$ "Success"), then Return (status, -1). 
Comment: Invoke the instantiate algorithm. Note that the entropy_input contains the nonce.

7. $(V$, Key, reseed_counter $)=$ HMAC_DRBG_Instantiate_algorithm (entropy_input , personalization_string).

Comment: Find an unused internal state.

8. (status, state_handle) $=$ Find_state_space $($ ) .

9. If (status $\neq$ "Success"), then Return (status, -1).

10. Save the initial state.

10.1 internal_state (state_handle). $V=V$.

10.2 internal_state (state_handle). Key $=$ Key.

10.3 internal_state (state_handle).reseed_counter $=$ reseed_counter.

10.4 internal_state (state_handle).security_strength = security_strength.

11. Return ("Success" and state_handle).

\section{HMAC_DRBG_Instantiate_algorithm:}

Input: bitstring (entropy_input, personalization_string).

Output: bitstring $(V, K e y)$, integer reseed_counter.

Process:

1. seed_material $=$ entropy_input $\|$ personalization_string.

2. Set Key to outlen bits of zeros.

3. Set $V$ to outlen $/ 8$ bytes of $0 x 01$.

4. $($ Key, $V)=$ HMAC_DRBG_Update (seed_material, Key, $V)$.

5. reseed_counter $=1$.

6. Return ( $V$, Key, reseed_counter $)$.

\section{B.2.2 Generating Pseudorandom Bits Using HMAC_DRBG}

The implementation returns a Null string as the pseudorandom bits if an error has been detected.

HMAC_DRBG_Generate_function:

Input: integer (state_handle, requested_no_of_bits, requested_security_strength).

Output: string (status), bitstring pseudorandom_bits.

Process:

Comment: Check for a valid state handle.

1. If $(($ state_handle $<0)$ or $($ state_handle $>2)$ or $($ internal_state $($ state_handle $)=\{$ Null, Null, 0,0$\}$ ), then Return ("State not available for the indicated state_handle", Null).

2. Get the internal state. 
$2.1 V=$ internal_state (state_handle). $V$.

2.2 Key $=$ internal_state (state_handle).Key.

2.3 security_strength $=$ internal_state $($ state_handle).security_strength .

2.4 reseed_counter $=$ internal_state $($ state_handle).reseed_counter.

Comment: Check the validity of the rest of the input parameters.

3. If (requested_no_of_bits > 7500), then Return ("Too many bits requested", Null).

4. If (requested_security_strength > security_strength), then Return ("Invalid requested_security_strength", Null).

Comment: Invoke the generate algorithm.

5. (status, pseudorandom_bits, $V$, Key, reseed_counter $)=$ HMAC_DRBG_Generate_algorithm $(V$, Key, reseed_counter, requested_number_of_bits).

6. If (status = "Reseed required"), then Return ("DRBG can no longer be used. A new instantiation is required", $N u l l)$.

7. Update the changed state values.

7.1 internal_state (state_handle). $V=V$.

7.2 internal_state (state_handle).Key $=$ Key.

7.3 internal_state (state_handle).reseed_counter=reseed_counter.

8. Return ("Success", pseudorandom_bits).

\section{HMAC_DRBG_Generate_algorithm:}

Input: bitstring $(V, K e y)$, integer (reseed_counter, requested_number_of_bits).

Output: string status, bitstring (pseudorandom_bits, V, Key), integer reseed_counter.

\section{Process:}

1 If (reseed_counter $\geq 10$ 000), then Return ("Reseed required", Null, V, Key, reseed_counter).

2. temp $=$ Null.

3 While $($ len $($ temp $)<$ requested_no_of_bits $)$ do:

\section{$3.1 V=$ HMAC $(K e y, V)$.}

3.2 temp $=$ temp $\| V$.

4. pseudorandom_bits $=$ leftmost $($ temp, requested_no_of_bits $)$.

5. $(K e y, V)=$ HMAC_DRBG_Update $(N u l l, K e y, V)$.

6. reseed_counter $=$ reseed_counter +1 .

7. Return ("Success", pseudorandom_bits, V, Key, reseed_counter). 


\section{B.3 CTR_DRBG Example Using a Derivation Function}

This example of CTR_DRBG uses AES-128 and uses the entire input block as the counter field. The reseed and prediction resistance capabilities are supported, and prediction resistance is obtained during every Get_entropy_input call and reseed request. Although the prediction_resistance_request parameter in the Get_entropy_input and reseed request could be omitted, in this case, they are shown in the pseudocode as a reminder that prediction_resistance will be performed. A block cipher derivation function using AES-128 is used, and a personalization string and additional input are supported. A total of five internal states are available. For this implementation, the functions and algorithms are written as separate routines. AES_ECB_Encrypt is the Block_Encrypt function (specified in Section 10.3.3) that uses AES128 in the ECB mode.

The nonce for instantiation (instantiation_nonce) consists of a 32-bit incrementing counter. The nonce is initialized when the DRBG is instantiated (e.g., by a call to the clock or by setting it to a fixed value) and is incremented for each instantiation.

The internal state contains the values for $V$, Key, reseed_counter, and security_strength, where $V$ and Key are bitstrings, and all other values are integers. Since prediction resistance is known to be supported, there is no need for prediction_resistance_flag in the internal state.

In accordance with Table 3 in Section 10.2.1, security strengths of 112 and 128 bits may be supported. Using AES-128, the following definitions are applicable for the instantiate, reseed and generate functions:

1. highest_supported_security_strength $=128$.

2. Input/output block length (blocklen $)=128$ bits.

3. Key length $($ keylen $)=128$ bits.

4. Required minimum entropy for the entropy input during instantiation and reseeding $=$ security_strength.

5. Minimum entropy input length $($ min_length $)=$ security_strength bits.

6. Maximum entropy input length $\left(\max \_\right.$length $)=1000$ bits.

7. Maximum personalization string input length (max_personalization_string_input_length) $=800$ bits.

8. Maximum additional input length (max_additional_input_length) $=800$ bits.

9. Seed length (seedlen) $=256$ bits.

10. Maximum number of bits per request (max_number_of_bits_per_request $)=4000$ bits.

11. Reseed interval (reseed_interval) $=100000$ requests.

\section{B.3.1 The CTR_DRBG_Update Function}

\section{CTR_DRBG_Update:}

Input: bitstring (provided_data,Key, V).

Output: bitstring (Key, V). 


\section{Process:}

1. temp $=$ Null.

2. While $($ len $(t e m p)<256)$ do

$$
2.1 V=(V+1) \bmod 2^{128} \text {. }
$$

2.2 output_block $=$ AES_ECB_Encrypt $(K e y, V)$.

2.3 temp $=$ temp $\|$ output_block.

3. temp $=$ leftmost $($ temp, 256$)$.

4 temp $=$ temp $\oplus$ provided_data.

5. Key $=$ leftmost (temp, 128).

6. $V=$ rightmost (temp, 128).

7. Return $(K e y, V)$.

\section{B.3.2 Instantiation of CTR_DRBG Using a Derivation Function}

This implementation will return a text message and an invalid state handle $(-1)$ when an error is encountered. Block_Cipher_df is the derivation function in Section 10.3.2, and uses AES-128 in the ECB mode as the Block_Encrypt function.

Note that this implementation does not include the prediction_resistance_flag in the input parameters, nor save it in the internal state, since prediction resistance is known to be supported.

\section{CTR_DRBG_Instantiate_function:}

Input: integer (requested_instantiation_security_strength), bitstring personalization_string.

Output: string status, integer state_handle.

\section{Process:}

Comment: Check the validity of the input parameters.

1. If (requested_instantiation_security_strength $>128$ ) then Return ("Invalid requested_instantiation_security_strength", -1).

2. If (len (personalization_string) > 800), then Return ("Personalization_string too long", -1).

3. If (requested_instantiation_security_strength $\leq 112$ ), then security_strength $=112$ Else security_strength $=128$.

\section{Comment: Get the entropy input.}

4. (status, entropy_input) $=$ Get_entropy_input (security_strength, security_strength, 1000,prediction_resistance_request).

5. If (status $\neq$ "Success"), then Return (status, -1 ). 
Comment: Increment the nonce; actual coding must ensure that the nonce wraps when its storage limit is reached, and that the counter pertains to all instantiations, not just this one.

6. instantiation_nonce $=$ instantiation_nonce +1 .

Comment: Invoke the instantiate algorithm.

7. $(V$, Key, reseed_counter $)=\mathbf{C T R}$ _DRBG_Instantiate_algorithm (entropy_input, instantiation_nonce, personalization_string).

Comment: Find an available internal state and save the initial values.

8. (status, state_handle) $=$ Find_state_space () .

9. If (status $\neq$ "Success"), then Return (status, -1 ).

10. Save the internal state.

10.1 internal_state_(state_handle) $\mathrm{V}=V$.

10.2 internal_state_(state_handle).Key $=$ Key.

10.3 internal_state_(state_handle).reseed_counter $=$ reseed_counter.

10.4 internal_state_(state_handle).security_strength = security_strength.

11. Return ("Success", state_handle).

\section{CTR_DRBG_Instantiate_algorithm:}

Input: bitstring (entropy_input, nonce, personalization_string).

Output: bitstring $(V, K e y)$, integer (reseed_counter).

Process:

1. seed_material $=$ entropy_input $\|$ nonce $\|$ personalization_string.

2. seed_material $=$ Block_Cipher_df (seed_material, 256).

3. Key $=0^{128}$. Comment: 128 bits.

4. $V=0^{128}$. Comment: 128 bits.

5. $($ Key, $V)=$ CTR_DRBG_Update (seed_material, Key, $V)$.

6. reseed_counter $=1$.

7. Return (V,Key, reseed_counter).

\section{B.3.3 Reseeding a CTR_DRBG Instantiation Using a Derivation Function}

The implementation is designed to return a text message as the status when an error is encountered.

CTR_DRBG_Reseed_function: 
Input: integer (state_handle), integer prediction_resistance_request, bitstring additional_input.

Output: string status.

Process:

Comment: Check for the validity of state_handle.

1. If $(($ state_handle $<0)$ or $($ state_handle $>4)$ or $($ internal_state $($ state_handle $)=\{$ Null, Null, 0,0$\}$ ), then Return ("State not available for the indicated state_handle").

2. Get the internal state values.

$2.1 V=$ internal_state (state_handle).V.

2.2 Key $=$ internal_state (state_handle).Key.

2.3 security_strength $=$ internal_state (state_handle).security_strength.

3. If (Ien (additional_input) > 800), then Return ("additional_input too long").

4. (status, entropy_input) = Get_entropy_input (security_strength, security_strength, 1000, prediction_resistance_request).

6. If (status $\neq$ "Success"), then Return (status).

Comment: Invoke the reseed algorithm.

7. $(V$, Key, reseed_counter $)=$ CTR_DRBG_Reseed_algorithm $(V, K e y$, reseed_counter, entropy_input, additional_input).

8. Save the internal state.

8.1 internal_state (state_handle). $V=V$.

8.2 internal_state (state_handle). Key $=$ Key.

8.3 internal_state (state_handle).reseed_counter $=$ reseed_counter.

8.4 internal_state (state_handle). security_strength = security_strength.

9. Return ("Success").

\section{CTR_DRBG_Reseed_algorithm:}

Input: bitstring $(V, K e y)$, integer (reseed_counter), bitstring (entropy_input, additional_input).

Output: bitstring $(V, K e y)$, integer (reseed_counter).

\section{Process:}

1. seed_material $=$ entropy_input $\|$ additional_input.

2. seed_material $=$ Block_Cipher_df (seed_material, 256).

3. $($ Key, $V)=$ CTR_DRBG_Update (seed_material, Key, V).

4. reseed_counter $=1$.

5. Return $(V, K e y$, reseed_counter $)$. 


\section{B.3.4 Generating Pseudorandom Bits Using CTR_DRBG}

The implementation returns a Null string as the pseudorandom bits if an error has been detected.

\section{CTR_DRBG_Generate_function:}

Input: integer (state_handle, requested_no_of_bits, requested_security_strength, prediction_resistance_request), bitstring additional_input.

Output: string status, bitstring pseudorandom_bits.

\section{Process:}

Comment: Check the validity of state_handle.

1. If $(($ state_handle $<0)$ or $($ state_handle $>4)$ or $($ internal_state $($ state_handle $)=\{$ Null, Null, 0,0$\}$ ), then Return ("State not available for the indicated state_handle", Null).

2. Get the internal state.

$2.1 V=$ internal_state (state_handle). $V$.

2.2 Key $=$ internal_state (state_handle).Key.

2.3 security_strength $=$ internal_state (state_handle).security_strength.

2.4 reseed_counter $=$ internal_state (state_handle).reseed_counter.

Comment: Check the rest of the input parameters.

3. If (requested_no_of_bits > 4000), then Return ("Too many bits requested", Null).

4. If (requested_security_strength > security_strength), then Return ("Invalid requested_security_strength", Null).

5. If (len (additional_input) > 800), then Return (“additional_input too long", Null).

6. reseed_required_flag $=0$.

7. If $(($ reseed_required_flag $=1) \mathrm{OR}($ prediction_resistance_flag $=1))$, then

7.1 status $=$ CTR_DRBG_Reseed_function (state_handle, prediction_resistance_request, additional_input).

7.2 If (status $\neq$ "Success"), then Return (status, Null).

7.3 Get the new working state values; the administrative information was not affected.

7.3.1 V=internal_state (state_handle).V.

7.3.2 Key =internal_state (state_handle).Key.

7.3.3 reseed_counter $=$ internal_state $($ state_handle).reseed_counter.

7.4 additional_input $=$ Null.

7.5 reseed_required_flag $=0$.

Comment: Generate bits using the generate algorithm. 
8. (status, pseudorandom_bits, $V$, Key, reseed_counter $)=$

CTR_DRBG_Generate_algorithm $(V$, Key, reseed_counter, requested_number_of_bits, additional_input).

9. If (status $=$ "Reseed required"), then

9.1 reseed_required_flag $=1$.

9.2 Go to step 7.

10. Update the internal state.

10.1 internal_state (state_handle). $V=V$.

10.2 internal_state (state_handle).Key $=$ Key.

10.3 internal_state (state_handle).reseed_counter $=$ reseed_counter.

10.4 internal_state (state_handle).security_strength = security_strength.

11. Return ("Success", pseudorandom_bits).

\section{CTR_DRBG_Generate_algorithm:}

Input: bitstring $(V, K e y)$, integer (reseed_counter, requested_number_of_bits) bitstring additional_input.

Output: string status, bitstring (returned_bits, $V$, Key), integer reseed_counter.

\section{Process:}

1. If (reseed_counter $>100$ 000), then Return ("Reseed required", Null, V, Key, reseed_counter).

2. If (additional_input $\neq N u l l)$, then

2.1 additional_input $=$ Block_Cipher_df (additional_input, 256).

$2.2(K e y, V)=$ CTR_DRBG_Update (additional_input, Key, $V)$.

Else additional_input $=0^{256}$.

3. temp $=$ Null.

4. While $(\operatorname{len}($ temp $)<$ requested_number_of_bits $)$ do:

$4.1 V=(V+1) \bmod 2^{128}$.

4.2 output_block $=$ AES_ECB_Encrypt $(K e y, V)$.

4.3 temp $=$ temp $\|$ output_block.

5. returned_bits $=$ leftmost $($ temp, requested_number_of_bits)

6. $(K e y, V)=$ CTR_DRBG_Update (additional_input, Key, V)

7. reseed_counter $=$ reseed_counter +1 .

8. Return ("Success", returned_bits, V, Key, reseed_counter). 


\section{B.4 CTR_DRBG Example Without a Derivation Function}

This example of CTR_DRBG is the same as the previous example except that a derivation function is not used (i.e., full entropy is always available). As in Appendix B.3, the CTR_DRBG uses AES-128. The reseed and prediction resistance capabilities are available. Both a personalization string and additional input are supported. A total of five internal states are available. For this implementation, the functions and algorithms are written as separate routines. AES_ECB_Encrypt is the Block_Encrypt function (specified in Section 10.3.3) that uses AES128 in the ECB mode.

The internal state contains the values for $V$, Key, reseed_counter, and security_strength, where $V$ and Key are strings, and all other values are integers. Since prediction resistance is known to be supported, there is no need for prediction_resistance_flag in the internal state.

In accordance with Table 3 in Section 10.2.1, security strengths of 112 and 128 bits may be supported. The definitions are the same as those provided in Appendix B.3, except that to be compliant with Table 3, the maximum size of the personalization_string is 256 bits. In addition, the maximum size of any additional_input is 256 bits (i.e., len (additional_input $\leq$ seedlen)).

\section{B.4.1 The CTR_DRBG_Update Function}

The update function is the same as that provided in Appendix B.3.1.

\section{B.4.2 Instantiation of CTR_DRBG Without a Derivation Function}

The instantiate function (CTR_DRBG_Instantiate_function) is the same as that provided in Appendix B.3.2, except for the following:

- Step 2 is replaced by:

If (len (personalization_string) > 256), then Return ("Personalization_string too long", $-1)$.

- $\quad$ Step 6 is replaced by :

instantiation_nonce $=$ Null.

The instantiate algorithm (CTR_DRBG_Instantiate_algorithm) is the same as that provided in Appendix B.3.2, except that steps 1 and $\overline{2}$ are replaced by:

temp = len (personalization_string).

If $\left(\right.$ temp < 256), then personalization_string $=$ personalization_string $\| 0^{256-t e m p}$. seed_material $=$ entropy_input $\oplus$ personalization_string.

\section{B.4.3 Reseeding a CTR_DRBG Instantiation Without a Derivation Function}

The reseed function (CTR_DRBG_Reseed_function) is the same as that provided in Appendix B.3.3, except that step 3 is replaced by:

If (len (additional_input) > 256), then Return (“additional_input too long”). 
The reseed algorithm (CTR_DRBG_Reseed_algorithm) is the same as that provided in Appendix B.3.3, except that steps 1 and 2 are replaced by:

temp $=$ len (additional_input).

If $\left(\right.$ temp <256), then additional_input $=$ additional_input $\| 0^{256-t e m p}$.

seed_material $=$ entropy_input $\oplus$ additional_input.

\section{B.4.4 Generating Pseudorandom Bits Using CTR_DRBG}

The generate function (CTR_DRBG_Generate_function) is the same as that provided in Appendix B.3.4, except that step 5 is replaced by:

If (Ien (additional_input) > 256), then Return ("additional_input too long", Null).

The generate algorithm (CTR_DRBG_Generate_algorithm) is the same as that provided in Appendix B.3.4, except that step 2.1 is replaced by:

temp $=$ len (additional_input).

If $($ temp $<256)$, then additional_input $=$ additional_input $\| 0^{256-t e m p}$. 


\section{Appendix C: (Informative) DRBG Mechanism Selection}

Almost no application or system designer starts with the primary purpose of generating good random bits. Instead, the designer typically starts with a goal that he wishes to accomplish, then decides on cryptographic mechanisms, such as digital signatures or block ciphers that can help him achieve that goal. Typically, as the requirements of those cryptographic mechanisms are better understood, he learns that random bits will need to be generated, and that this must be done with great care so that the cryptographic mechanisms will not be weakened. At this point, there are three things that may guide the designer's choice of a DRBG mechanism:

a. He may already have decided to include a set of cryptographic primitives as part of his implementation. By choosing a DRBG mechanism based on one of these primitives, he can minimize the cost of adding that DRBG mechanism. In hardware, this translates to lower gate count, less power consumption, and less hardware that must be protected against probing and power analysis. In software, this translates to fewer lines of code to write, test, and validate.

For example, a module that generates RSA signatures has an available hash function, so a hash-based DRBG mechanism (e.g., Hash_DRBG or HMAC_DRBG) is a natural choice.

b. He may already have decided to trust a block cipher, hash function, or keyed hash function to have certain properties. By choosing a DRBG mechanism based on similar properties, he can minimize the number of algorithms he has to trust.

For example, an AES-based DRBG mechanism (i.e., CTR_DRBG using AES) might be a good choice when a module also provides encryption with AES. Since the security of the module is dependent on the strength of AES, the module's security is not made dependent on any additional cryptographic primitives or assumptions.

c. Multiple cryptographic primitives may be available within the system or consuming application, but there may be restrictions that need to be addressed (e.g., code size or performance requirements).

For example, a module with support for both hash functions and block ciphers might use the CTR_DRBG if the ability to parallelize the generation of random bits is needed.

The DRBG mechanisms specified in this Recommendation have different performance characteristics, implementation issues, and security assumptions.

\section{C.1 Hash_DRBG}

Hash_DRBG is based on the use of an approved hash function in a counter mode similar to the counter mode specified in [SP 800-38A]. For each generate request, the current value of $V$ (a secret value in the internal state) is used as the starting counter that is iteratively changed to generate each successive outlen-bit block of requested output, where outlen is the number of bits in the hash function output block. At the end of the generate request, and before the pseudorandom output is returned to the consuming application, the secret value $V$ is updated in order to prevent backtracking. 
Performance. The Generate function is parallelizable, since it uses the counter mode. Within a generate request, each outlen-bit block of output requires one hash function computation and several addition operations; an additional hash function computation is required to provide the backtracking resistance. Hash_DRBG produces pseudorandom output bits in about half the time required by $\mathbf{H M A C}$ _DRBG.

Security. Hash_DRBG's security depends on the underlying hash function's behavior when processing a series of sequential input blocks. If the hash function is replaced by a random oracle, Hash_DRBG is secure. It is difficult to relate the properties of the hash function required by $\mathbf{H a s h}$ _DRBG with common properties, such as collision resistance, pre-image resistance, or pseudorandomness. There are known problems with Hash_DRBG when the DRBG is instantiated with insufficient entropy for the requested security strength, and then later provided with enough entropy to attain the amount of entropy required for the security strength, via the inclusion of additional input during a generate request. However, these problems do not affect the DRBG's security when Hash_DRBG is instantiated with the amount of entropy specified in this Recommendation.

Constraints on Outputs. As shown in Table 2 of Section 10.1, for each hash function, up to $2^{48}$ generate requests may be made, each of up to $2^{19}$ bits.

Resources. Hash_DRBG requires access to a hash function, and the ability to perform addition with seedlen-bit integers. Hash_DRBG uses the hash-based derivation function Hash_df (specified in Section 10.3.1) during instantiation and reseeding. Any implementation requires the storage space required for the internal state (see Section 10.1.1.1).

Algorithm Choices. The choice of hash functions that may be used by Hash_DRBG is discussed in Section 10.1.

\section{C.2 HMAC_DRBG}

HMAC_DRBG is built around the use of an approved hash function using the HMAC construction. To generate pseudorandom bits from a secret key $(K e y)$ and a starting value $V$, the HMAC_DRBG computes

$$
V=\text { HMAC }(K e y, V) \text {. }
$$

At the end of a generation request, the HMAC_DRBG generates a new Key and $V$, each requiring one HMAC computation.

Performance. HMAC_DRBG produces pseudorandom outputs considerably more slowly than the underlying hash function processes inputs; for SHA-256, a long generate request produces output bits at about $1 / 4$ of the rate that the hash function can process input bits. Each generate request also involves additional overhead equivalent to processing 2048 extra bits with SHA256. Note, however, that hash functions are typically quite fast; few if any consuming applications are expected to need output bits faster than HMAC_DRBG can provide them.

Security. The security of HMAC_DRBG is based on the assumption that an approved hash function used in the HMAC construction is a pseudorandom function family. Informally, this means that when an attacker does not know the key used, HMAC outputs look random, even given knowledge and control over the inputs. In general, even relatively weak hash functions seem to be quite strong when used in the HMAC construction. On the other hand, there is not a 
reduction proof from the hash function's collision resistance properties to the security of the DRBG; the security of HMAC_DRBG ultimately relies on the pseudorandomness properties of the underlying hash function. Note that the pseudorandomness of HMAC is a widely used assumption in designs, and the HMAC_DRBG requires far less demanding properties of the underlying hash function than Hash_DRBG.

Constraints on Outputs. As shown in Table 2 of Section 10.1, for each hash function, up to $2^{48}$ generate requests may be made, each of up to $2^{19}$ bits.

Resources. HMAC_DRBG requires access to a dedicated HMAC implementation for optimal performance. However, a general-purpose hash function implementation can always be used to implement HMAC. Any implementation requires the storage space required for the internal state (see Section 10.1.2.1).

Algorithm Choices. The choice of hash functions that may be used by HMAC_DRBG is discussed in Section 10.1.

\section{C.3 CTR_DRBG}

CTR_DRBG is based on using an approved block cipher algorithm in counter mode (see [SP 800-38A]). At the present time, only three-key TDEA and AES are approved for use by the Federal government for use in this DRBG mechanism. Pseudorandom outputs are generated by encrypting successive values of a counter; after a generate request, a new key and new starting counter value are generated.

Performance. For large generate requests, CTR_DRBG produces outputs at the same speed as the underlying block cipher algorithm encrypts data. Furthermore, CTR_DRBG is parallelizable. At the end of each generate request, work equivalent to two, three or four encryptions is performed, depending on the choice of underlying block cipher algorithm, to generate new keys and counters for the next generate request.

Security. The security of CTR_DRBG is directly based on the security of the underlying block cipher algorithm, in the sense that, as long as some limits on the total number of outputs are observed, any attack on CTR_DRBG represents an attack on the underlying block cipher algorithm.

Constraints on Outputs. As shown in Table 3 of Section 10.2.1, for each of the three AES key sizes, up to $2^{48}$ generate requests may be made, each of up to $2^{19}$ bits, with a negligible chance of any weakness that does not represent a weakness in AES. However, the smaller block size of TDEA imposes more constraints: each generate request is limited to $2^{13}$ bits, and at most, $2^{32}$ such requests may be made.

The output constraints are necessary to avoid a distinguishing attack on the CTR_DRBG, described in [Campagna], in which the fact that a single generate call can never produce a duplicate block from the block cipher is used to build a distinguisher for the DRBG's outputs. These output constraints apply to the use of CTR_DRBG for any single purpose, regardless of how many times the DRBG is reseeded. However, the distinguishing attack is theoretical - it poses no practical threat to any real-world application of the DRBG. 
The distinguishing attack is conceptually quite simple. For concreteness, consider the case of TDEA CTR_DRBG. The DRBG generates a maximum of 128 64-bit blocks per generate request, thus providing $2^{13}$ bits per request. An ideal random source would have a very small probability (about $2^{-51}$ ) of producing a pair of identical 64-bit blocks within that generate request output; each generate request from the CTR_DRBG is generated by running the block cipher in counter mode, so there can never be a duplicate block produced within a generate request output. (The block cipher is rekeyed between generate requests, so duplicate blocks can appear in different generate request outputs.) TDEA CTR_DRBG permits the use of up to $2^{32}$ generate requests. An ideal random source, providing $2^{32}$ sequences of 128 64-bit blocks, would have a probability of about $2^{-19}$ of having a duplicate block in one of those sequences of 128 64-bit blocks; the CTR_DRBG will never have such a duplicate block. This provides a distinguisher an attacker, given a sequence of $2^{13} 2^{32}=2^{45}$ bits from an ideal random source, has about a $2^{-19}$ probability of seeing an event happen that could never happen from TDEA CTR_DRBG.

Consider some application in which a DRBG's outputs must not be distinguishable by an attacker, and assume that an attacker who sees $2^{64}$ bits of output from the TDEA CTR_DRBG across at least one reseed, and wants to decide whether these bits came from the CTR_DRBG or from an ideal random source. The best case for the attacker is that each generate request used the maximum allowed value of $2^{13}$ bits of output $=12864$-bit blocks of output. In this case, the TDEA CTR_DRBG received $2^{45}$ generate requests. An ideal random sequence has a probability of about $2^{-6}$ of having a duplicate block in one of the generate outputs; the CTR_DRBG outputs will never have one. An attacker looking at the sequence will not be able to determine that it came from the CTR_DRBG, though he would have a pretty large advantage in a distinguishing game.

The case for AES CTR_DRBG is similar: each generate request may produce no more than $2^{19}$ bits, which means $2^{12} 128$-bit blocks. In an ideal random sequence of $2^{12} 128$-bit blocks, the probability that any two blocks will be the same is approximately $2^{-105}$; AES CTR_DRBG will never provide a generate output with duplicate blocks. AES CTR_DRBG permits up to $2^{48}$ generate requests, so an attacker seeing the maximum length of output permitted $\left(2^{67}\right.$ bits $)$ from either an AES CTR_DRBG instance or an ideal random sequence will have a $2^{-57}$ probability of being able to distinguish the two.

Resources. CTR_DRBG may be implemented with or without a derivation function.

When a derivation function is used, CTR_DRBG can process the personalization string and any additional input in the same way as any other DRBG mechanism, but at a cost in performance because of the use of the derivation function (as opposed to not using the derivation function; see below). Such an implementation may be seeded by any approved randomness source that may or may not provide full entropy.

When a derivation function is not used, CTR_DRBG is more efficient when the personalization string and any additional input are provided, but is less flexible because the lengths of the personalization string and additional input cannot exceed seedlen bits. Such implementations must be seeded by a randomness source that provides full entropy (e.g., an approved entropy source that has full entropy output or an approved NRBG).

CTR_DRBG requires access to a block cipher algorithm, including the ability to change keys, and the storage space required for the internal state (see Section 10.2.1.1). 
Algorithm Choices. The choice of block cipher algorithms and key sizes that may be used by CTR_DRBG is discussed in Section 10.2.1.

\section{C.4 Summary for DRBG Selection}

Table C-1 provides a summary of the costs and constraints of the DRBG mechanisms in this Recommendation.

Table C-1: DRBG Mechanism Summary

\begin{tabular}{|c|c|c|}
\hline & $\begin{array}{c}\text { Dominating } \\
\text { Cost/Block }\end{array}$ & $\begin{array}{c}\text { Constraints } \\
\text { (max.) }\end{array}$ \\
\hline Hash_DRBG & 2 hash function calls & $2^{48}$ calls of $2^{19}$ bits \\
\hline HMAC_DRBG & 4 hash function calls & $2^{48}$ calls of $2^{19}$ bits \\
\hline CTR_DRBG (TDEA) & 1 TDEA encrypt & $2^{32}$ calls of $2^{13}$ bits \\
\hline CTR_DRBG (AES) & 1 AES encrypt & $2^{48}$ calls of $2^{19}$ bits \\
\hline
\end{tabular}




\section{Appendix D : (Informative) References}

[FIPS 140] Federal Information Processing Standard (FIPS) 140-2, Security Requirements for Cryptographic Modules, May 25, 2001 (including Change Notices as of December 3, 2002).

http://csrc.nist.gov/publications/fips/fips140-2/fips1402.pdf [accessed 6/9/15].

[FIPS 180] Federal Information Processing Standard (FIPS) 180-4, Secure Hash Standard (SHS), March 2012.

http://csrc.nist.gov/publications/fips/fips180-4/fips-180-4.pdf [accessed 6/9/15].

[FIPS 197] Federal Information Processing Standard (FIPS) 197, Advanced Encryption Standard (AES), November 2001.

http://csrc.nist.gov/publications/fips/fips197/fips-197.pdf [accessed 6/9/15].

[FIPS 198] Federal Information Processing Standard (FIPS) 198-1, The Keyed-Hash Message Authentication Code (HMAC), July 2008. http://csrc.nist.gov/publications/fips/fips198-1/FIPS-198-1 final.pdf [accessed 6/9/15].

[SP 800-38A] National Institute of Standards and Technology Special Publication (SP) 80038A, Recommendation for Block Cipher Modes of Operation: Methods and Techniques, December 2001.

http://csrc.nist.gov/publications/nistpubs/800-38a/sp800-38a.pdf [accessed 6/9/15].

[SP 800-38D] National Institute of Standards and Technology Special Publication (SP) 80038D, Recommendation for Block Cipher Modes of Operation: Galois/Counter Mode (GCM) and GMAC, November 2007. http://csrc.nist.gov/publications/nistpubs/800-38D/SP-800-38D.pdf [accessed 6/9/15].

[SP 800-57] NIST Special Publication (SP) 800-57 Part 1 Revision 3, Recommendation for Key Management-Part 1: General, July 2012.

http://csrc.nist.gov/publications/nistpubs/800-57/sp80057 part1_rev3_general.pdf [accessed 6/9/15].

[SP 800-67] NIST Special Publication (SP) 800-67 Revision 1, Recommendation for the Triple Data Encryption Algorithm (TDEA) Block Cipher, January 2012. http://csrc.nist.gov/publications/nistpubs/800-67-Rev1/SP-800-67-Rev1.pdf [accessed 6/9/15].

[SP 800-90B] NIST Special Publication (SP) 800-90B (Draft), Recommendation for the Entropy Sources Used for Random Bit Generation, August 2012 [re-released September 2013]. http://csrc.nist.gov/publications/drafts/800-90/draft-sp800-90b.pdf [accessed 6/9/15].

[SP 800-90C] NIST Special Publication (SP) 800-90C (Draft), Recommendation for Random Bit Generator (RBG) Constructions, August 2012 [re-released September 2013]. 
http://csrc.nist.gov/publications/drafts/800-90/draft-sp800-90c.pdf [accessed 6/9/15].

[SP 800-107] NIST Special Publication (SP) 800-107 Revision 1, Recommendation for Applications Using Approved Hash Algorithms, August 2012. http://csrc.nist.gov/publications/nistpubs/800-107-rev1/sp800-107-rev1.pdf [accessed 6/9/15].

[Campagna] M. J. Campagna, Security Bounds for the NIST Codebook-based Deterministic Random Bit Generator, Report 2006/379, Cryptology ePrint Archive, November 2006. http://eprint.iacr.org/2006/379 [accessed 6/9/15]. 


\section{Appendix E : (Informative) Revisions}

The original version of this Recommendation was completed in June, 2006. In March 2007, the following changes were made (note that the changes are indicated in italics):

1. Section 8.3, item 1.a originally stated the following:

"One or more values that are derived from the seed and become part of the internal state; these values must usually remain secret"

The item now reads:

"One or more values that are derived from the seed and become part of the internal state; these values should remain secret".

2. In Section 8.4, the third sentence originally stated:

"Any security strength may be requested, but the DRBG will only be instantiated to one of the four security strengths above, depending on the DRBG implementation."

The sentence now reads:

"Any security strength may be requested (up to a maximum of 256 bits), but the DRBG will only be instantiated to one of the four security strengths above, depending on the DRBG implementation."

3. In Section 8.7.1, the list of examples of information that could appear in a personalization string included private keys, PINs and passwords. These items were removed from the list, and seedfiles were added.

4. In Section 10.3.1.4, a step was inserted that will provide backtracking resistance (step 14 of the pseudocode). The same change was made to the example in Appendix B.5.3 (step 19.1). In addition, the two occurrences of block_counter (in input 1 and processing step 1) were corrected to be reseed_counter.

This Recommendation was developed in concert with American National Standard (ANS) X9.82, a multi-part standard on random number generation. Many of the DRBGs in this Recommendation and the requirements for using and validating them are also provided in ANS X9.82, Part 3. Other parts of that Standard discuss entropy sources and RBG construction. During the development of the latter two documents, the need for additional requirements and capabilities for DRBGs were identified. As a result, the following changes were made to this Recommendation in August 2008:

1. Definitions have been added in Section 4 for the following: approved entropy source, DRBG mechanism, fresh entropy, ideal random bitstring, ideal random sequence and secure channel. The following definitions have been modified: backtracking resistance, deterministic random bit generator (DRBG), entropy, entropy input, entropy source, full entropy, min-entropy, prediction resistance, reseed, security strength, seed period and source of entropy input.

2. In Section 6, a link was provided to examples for the DRBGs specified in this Recommendation. 
3. In Section 7.2, paragraph 3. $2^{\text {nd }}$ sentence: The "should" has been changed to "shall", so that the sentence now reads:

The personalization string shall be unique for all instantiations of the same DRBG mechanism type (e.g., HMAC_DRBG).

4. In Section 8.2, paragraph 2, additional text was added to the first sentence, which now reads:

A DRBG is instantiated using a seed and may be reseeded; when reseeded, the seed shall be different than the seed used for instantiation.

5. In Section 8.5, Figure 4 has been updated, and the last paragraph has been revised to discuss the use of a secure channel.

6. In Sections 8.6.5 and 8.6.9, statements were inserted that prohibit a DRBG instantiation from reseeding itself.

7. References to "entropy input" have been removed from Section 8.6.9.

8. Section 8.8: An example was added to further clarify the meaning of prediction resistance.

9. In Section 9, a prediction_resistance_request parameter has been added to the Get_entropy_input call, along with a description of its purpose to the text underneath the call.

10. In Section 9, a footnote was inserted to explain why a prediction_resistance_request parameter may be useful in the Get_entropy_input call.

11. In Section 9.1, the following changes were made:

- The following sentence has been added to the description of the prediction_resistance_flag:

In addition, step 6 can be modified to not perform a check for the prediction_resistance_flag when the flag is not used in an implementation; in this case, the Get_entropy_input call need not include the prediction_resistance_request parameter.

- The following requirement has been added to the Required information not provided by the consuming application during instantiation:

This input shall not be provided by the consuming application as an input parameter during the instantiate request.

- A prediction_resistance_request parameter has been added to the Get_entropy_input call of step 6 of the Instantiate Process.

- Step 5 was originally intended for implementations of the Dual_EC_DRBG to select an appropriate curve. This function is now performed by the Dual_EC_DRBG's Instantiate_algorithm. Changes were made to provide the security strength to the Instantiate_algorithm. The Instantiate_algorithm for each DRBG was changed to allow the input of the security strength.

12. In Section 9.2, the following changes have been made: 
- A prediction_resistance_request parameter has been added to the Reseed_function call.

- A description of the parameter has been added below the function call.

- A step was inserted that checked a request for prediction resistance (via the prediction_resistance_request parameter) against the state of the prediction_resistance_flag that may have been set during instantiation.

- A prediction_resistance_request parameter has been added to the Get_entropy_input call of (newly numbered) step 4 of the Reseed Process.

- In the description of the entropy_input parameter, a restriction was added that the entropy_input is not to be provided by the instantiation being reseeded.

- A footnote was inserted to explain why the prediction_resistance_request parameter might be useful.

13. In Section 9.3.1, the following changes were made:

- Text has been added to item 4 to refer to the Reseed_function.

- A prediction_resistance_request parameter has been added to the Get_entropy_input call of step 7.1 of the Generate Process.

- A substep was inserted in step 9 of the Generate Process to check the prediction_resistance request against the state of the prediction_resistance_flag.

14. In Section 9.3.2, step e, a phrase addressing the presence of the prediction_resistance_request indicator was inserted.

15. In Sections 10.1 and 10.3.1, the new hash functions approved in FIPS 180-4 have been added.

16. In Sections 10.1.2 (HMAC_DRBG) and 10.2.1 (CTR_DRBG), the update functions have been renamed to reflect the DRBG with which they are associated (i.e., renamed to HMAC_DRBG_Update and CTR_DRBG_Update).

17. In Section 10.1.2.1, the last paragraph has been revised to indicate that only the Key is considered to be a critical value.

18. In Sections 10.1.2.3, 10.2.1.3.1, 10.2.1.3.2 and 10.3.1.2, the description of the personalization_string has been revised to indicate that the length the personalization_string may be zero.

19. In Section 10.2.1.5, the following statement has been added to the first paragraph:

If the derivation function is not used, then the maximum allowed length of additional_input $=$ seedlen.

20. In Section 10.3.1.2, the specification was changed to select an elliptic curve and return the parameters of that curve to the Instantiate_function that called the routine.

21. In the first paragraph of Appendix A.1, a statement has been added that if alternative points are desired, they shall be generated as specified in Appendix A.2. 
22. The original Appendices $\mathrm{C}$ and $\mathrm{D}$ on entropy sources and RBG constructions, respectively, have been removed and the topics will be discussed in SP 800-90B and C

23. In Appendix C.2 (originally Appendix E.2), a paragraph has been inserted after the table of E values that discusses the analysis associated with the table values.

24. The additional uses of the prediction_resistance_request parameter (as specified in Section 9) have been added to the following appendices:

- D.1.1, step 4;

- D.1.2, Input and step 4;

- D.1.3, step 7.1;

- D.3.2, step 4;

- D.3.3, Input and step 4; and

- D.3.4, step 7.1.

25. The name of the update call has been changed in the following appendices:

- D.2.1, step 4;

- D.2.2, step 5;

- D.3.1, title; and

- D.4.1, title.

26. In Appendix D.3 (originally Appendix F.3), the first paragraph, which discusses the example, has been modified to discuss the prediction_resistance_request parameter in the Get_entropy_input call.

27. In Appendix D.5 (originally Appendix F.5), the description of the example in paragraph 2 has been changed so that the example does not include prediction resistance, and the definition for the reseed interval has been removed from the list. The Dual_EC_Instantiate_function has been modified to reflect the changes made to the Instantiate_function and Instantiate_algorithm (see the last bullet of modification 8 above). In addition, the pseudocode for the Reseed_function has been removed, and steps in F.5.1 and F.5.2 that dealt with reseeding have been removed.

In June 2015, the following substantive changes were made in Revision 1 of [SP 800-90A]:

1. The following definitions were modified to be consistent with definitions in other parts of this Recommendation: backtracking resistance, entropy source, non-deterministic random bit generator, prediction resistance, and source of entropy input. The following definitions have been removed: public key and public-key pair. A definition for "randomness source" has been added, and the definition of "source of entropy input" has been removed.

2. The term "source of entropy input" has been replaced by "randomness source" to avoid confusion with the term "entropy source input," which is used in SP 800-90C to mean input from an entropy source. A "randomness source" (formerly "source of entropy input") could be an entropy source, an NRBG or a DRBG. 
3. Section 5: The ECDLP abbreviation and the floor and ged symbols were removed. Definitions of the leftmost, rightmost, min and select functions have been added, and have been used throughout the document.

4. Section 6: The reference to number-theoretic problems was removed, as well as the old Appendix A that provided security considerations for DRBGs based on elliptic curves, and the old Appendix F that listed shall statements.

5. Section 7: The first paragraph has been modified, and includes an additional shall statement. In Section 7.1, the first two sentences have been modified for clarity. In Section 7.2, the second paragraph and the first sentence of the third paragraph have been modified for clarity; the personalization string is now recommended, rather than required, to be unique. In Section 7.4, the second item has been modified for clarity, and the last paragraph has been removed, since it was not needed here.

6. Section 8: In Section 8.1, the second sentence has been modified for clarity. In Section 8.2, additional text has been added to the last sentence for clarity. In Section 8.3, item 1b, the reference to blocks was removed, since it pertained to the Dual_EC_DRBG. In Section 8.4, the third sentence is a general statement that replaces the last two sentences of that paragraph; the subject with more detail is now discussed below Table 1. In the paragraph under Figure 4, text has been inserted in the second sentence for clarity. The first sentence of the next paragraph has been modified for clarity, and an additional paragraph has been added to the section to mention the relationship between a DRBG sub-boundary and a cryptographic module boundary.

7. Section 8.5: A reference to the cryptographic boundary for FIPS 140 has been inserted in bold to draw the reader's attention to the fact that it is different than the DRBG's boundaries. In the paragraph under item 3 , an example has been provided for clarity. In the following two paragraphs, a reference to SP 800-90C has been inserted to direct the reader to that document for further discussion on cryptographic module boundaries.

8. Section 8.6: In Section 8.6.2, a reference to fresh entropy has been inserted in the second sentence. In Section 8.6.3, text has been inserted at the end of the second sentence for clarity. In Section 8.6.4, a shall statement has been inserted at the end of the first sentence. Sections 8.6.5 and 8.6.7 were revised to clarify the source of the entropy input and nonce. In Section 8.6.6, text was inserted that states that entropy input is a critical security parameter for cryptographic module validation. Section 8.6.7 was modified to provide more information about suitable nonces and to state that the uniqueness of the nonce is applicable to the cryptographic module in which it is used, and to indicate that the nonce is a critical security parameter. In Section 8.6.8, text was added about enforcing the seedlife. In Section 8.6.9, 'DRBG' was changed to 'DRBG instantiation' for clarity.

9. Section 8.7: Sections 8.7.1 and 8.7.2 have been modified to clarify that the optional personalization string and additional input may be obtained from outside a cryptographic module, that the personalization string is not a critical security parameter, and that the additional input may be a critical security parameter if secret information is included.

10. Section 8.8: The last sentence of the second paragraph under the list has 'direct or indirect' inserted for clarity. A paragraph has been added to the end of the section to recommend reseeding whenever possible. 
11. Section 9: A paragraph discussing the pseudocode used has been inserted at the beginning of the section, and modifications to the third and fourth paragraphs have been made for clarity; text has also been added to the next-to-last paragraph that discusses error codes more thoroughly. The last sentence in the third paragraph has been modified to only require that the entropy input and nonce be provided as discussed in Sections 8.6.5 and 8.6.7 and in SP 800-90C. A paragraph has been added to discuss checking the status code. In Section 9.2, clarifying information has been inserted about the prediction_resistance_request parameter. In Sections 9.1, 9.2 and 9.3, returns to the consuming application have been modified for those cases where other than SUCCESS is appropriate as a status to be returned from the function (e.g., parameter errors, entropy unavailability or entropy source failure); this change was made to better accommodate the various Get_entropy_input constructions specified in SP 800-90C. In Section 9.1 and 9.3.1, the item in the list referring to elliptic-curve parameters was removed, and the discussion of the status output has been modified for clarity.

12. Section 10: Section 10 now includes a link to the DRBG test vectors on the NIST web site.

Sections 10.1, 10.1.1 and 10.1.2 now include short discussions about selecting hash functions to support the DRBG's intended security strength.

The Dual_EC_DRBG has been removed, and section numbers adjusted accordingly. In Section 10.2.1, a paragraph under Table 3 has been added for explanatory purposes. In Section 10.2.1.3.2, the first paragraph has been modified for clarity. Section 10.2 has been modified to allow the counter field to be a subset of the input block and to allow either derivation function specified in the document; this is indicated in step 2.1 of Section 10.2.1.2 and step 4.1 of Sections 10.2.1.5.1 and 10.2.1.5.2 (note that this change continues to allow the use of the entire input block as the counter field, as was specified in the previous versions of this document); Table 3 has been modified to include restrictions on the length of the counter field and to indicate the restrictions on the number of bits that can be requested during a single request as a function of the counterfield length and the previous restriction on the number of bits that could be requested. The first paragraphs of Sections 10.3 and 10.3.2 have been modified slightly for clarity.

Step 11 in Section 10.3.2 has been respecified using the (new) select function.

13. Section 11: The third paragraph has been added for clarity, and the last sentence of the next paragraph has been removed. In Section 11.1, the references to the Dual_EC_DRBG have been removed from the third and fifth bullet, and the wording of the next-to-last bullet has been modified to be conditional. In Section 11.2, additional text has been inserted to address validation testing. In Section 11.3, the health testing requirements have been modified.

14. The previous Appendix A was removed; this appendix contained application-specific constants for the Dual_EC_DRBG.

15. Appendix A now contains the conversion routines. Appendix A.5.4 (the old Appendix B.5.4), which contained the complex modular method for converting bits to numbers, has been removed because of an error in the specification. 
16. Appendix B now contains the pseudocode examples previously provided in Appendix D, less examples for the Dual_EC_DRBG. In Appendix B.4, the discussion of the example has been changed slightly.

17. The previous Appendix $\mathrm{C}$ was removed; this appendix contained security considerations relating to the Dual_EC_DRBG.

18. The new Appendix $\mathrm{C}$ is the same as the previous Appendix E, minus the Dual_EC_DRBG discussion.

Additional text has been inserted into the discussion of the CTR_DRBG in Appendix C. 3 (the constraints subsection) that discusses the constraints provided in Table 3 of Section 10.2.1.

19. The referenced documents now in Appendix D have been updated, and a reference to [Campagna] has been added.

20. The previous Appendix F was removed; this appendix contained a list of shall statements that could not be validated by NIST's validation program. 